\title{
CONTRIBUCIÓN AL CONOCIMIENTO DE LA COMPOSICIÓN FLORÍSTICA DEL DEPARTAMENTO DE HUÁNUCO, PERÚ
}

\author{
Ricardo ZÁRATE ${ }^{1}$, Tony J. MORI ${ }^{1}$, Nandy L. MACEDO ${ }^{2}$, George P. GALLARDO ${ }^{1}$, Manuel FLORES ${ }^{3}$, \\ Percy MARTÍNEZ ${ }^{1}$, Fredy F. RAMÍREZ ${ }^{4}$, Luis A. TORRES ${ }^{5}$ \\ 1 Instituto de Investigaciones de la Amazonía Peruana. Programa de Investigación en Cambio Climático Desarrollo \\ Territorial y Ambiente (Proterra); Av. Quiñones km 2.5, San Juan Bautista, Maynas, Loreto, Perú. rzarate@iiap.org.pe \\ 2. Instituto de Investigaciones de la Amazonía Peruana. Programa de Investigación de Biodiversidad Amazónica; Av. \\ Quiñones km 2.5, San Juan Bautista, Maynas, Loreto, Perú. \\ 3. Universidad Nacional de la Amazonía Peruana. Facultad de Ciencias Biológicas. Jr. Pebas cuadra 5, Iquitos, Loreto, Perú. \\ 4. Universidad Nacional de la Amazonía Peruana. Facultad de Ciencias Forestales. Jr. Pebas cuadra 5, Iquitos, Loreto, Perú. \\ 5. Servicios de Biodiversidad. Jr. Independencia 405. Punchana, Maynas, Loreto, Perú.
}

\section{RESUMEN}

Realizamos un inventario florístico de las plantas del departamento de Huanuco, Perú basado en 30 unidades de muestreo ( 9 parcelas de 50 × $20 \mathrm{~m}, 4$ parcelas de 50 x $10 \mathrm{~m}$ y 17 trayectos de 10 a $80 \mathrm{~m}$ de largo). Se reportó un total de 1423 individuos que corresponde a 756 especies ( 354 especies y 402 morfoespecies), en 403 géneros y 130 familias. Uniendo nuestros resultados con la información publicada por Bracko \& Zaruchi (1993), Arévalo (1998), Ulloa et al. (2004), Salinas (2005), León et al. (2006), Salvador et al. (2006), Cachique (2009), Castillo (2009), Salvador et al. (2009) y otros, se reporta para el departamento de Huánuco 4712 especies incluidas en 1443 géneros y 226 familias de plantas (Licofitas, Pteridofitas, Gimnospermas y Angiospermas). Los resultados de estos inventarios florísticos muestran que el departamento de Huánuco es uno más diversos del Perú en especies de plantas junto con Loreto, Junín y Cuzco.

PALABRAS CLAVE: Angiospermas, Flora de Huánuco, Gimnospermas, Licofitas, Pteridofitas.

\section{CONTRIBUTION TO THE KNOWLEDGE OF THE FLORISTIC COMPOSITION OF THE DEPARTMENT OF HUANUCO, PERU}

\begin{abstract}
A floristic inventory in the Department of Huanuco, Peru, was made based on 30 samples units (nine $50 \mathrm{x}$ $20 \mathrm{~m}$ plots; four $50 \times 10 \mathrm{~m}$ plots, and 17 transects $10-80 \mathrm{~m}$ long). A total of 1423 individuals corresponding to 756 species (354 species and 402 morphospecies), 403 genera and 130 families were reported. Our results together with the information published by Bracko \& Zaruchi (1993), Arévalo (1998), Ulloa et al. (2004), Salinas (2005), León et al. (2006), Salvador et al. (2006), Cachique (2009), Castillo (2009) Salvador et al. (2009) and others, reports 4712 species for the department of Huanuco, includes in 1443 genera and 226 families of plants (Lycophytes, Pteridophytes, Gymnosperms and Angiosperms). These results show that the Department of Huanuco is one of the most diverse regions in Peru along with Loreto, Junin and Cuzco.
\end{abstract}

KEYWORDS: Angiosperms, Flora of Huanuco, Gymnosperms, Lycophytes, Pteridophytes. 


\section{INTRODUCCIÓN}

La composición florística se define como las familias, géneros y especies que habitan en un lugar o área determinada (Font Quer, 1985), en relación a factores bióticos (ocurrencia de claros, ecología de las especies, disponibilidad de semillas, diversidad y otros) y abióticos (clima, altitud, precipitación, viento, suelos, topografía y otros), Shimwell (1971). Conocer la composición florística es importante para el manejo sostenible de los recursos vegetales.

Los primeros estudios de la flora del departamento de Huánuco estan representados por los trabajos de Hipólito Ruiz y José A. Pavon, Antonio Raimondi, Augusto Weberbauer, y otros. Mientras que recientemente tenemos a Brako \& Zaruchi (1993), Ulloa et al. (2004), León et al. (2006), Rodríguez et al. 2006, y Salvador et al. (2006). Asimismo, existen varias publicaciones para grupos taxonómicos específicos dentro del departamento, como: Arévalo (1998) para las Pteridofitas, Salinas (2005) para el orden Scrophulariales, Salvador et al. (2009) para genero Carex, Castillo (2009) para la familia Rubiaceae, Gonzáles (2012) para la familia Plantaginaceae y García (2013) para familia Melastomataceae. Del mismo modo, Cachique (2009) publicó la diversidad de las plantas epífitas del Jardín Botánico de la Universidad Nacional Agraria de la Selva; Moreno et al. (2007) publico sobre las Fabaceae potencialmente útiles de la provincia de Huánuco y Vidal \& Ramos (2008) sobre la utilización de algunas plantas.

Aunque existen estudios que han logrado incrementar la información del conocimiento de la composición florística del departamento de Huánuco, falta un documento que reuna toda la información disponible al respecto. Este es un primer esfuerzo de sistematizar la información existente, lo cuál nos ayudará a identificar los vacíos de información florística y al mismo tiempo evidenciar muchas especies que están esperando ser descubiertas. Por lo que el objetivo del presente trabajo fue actualizar la información sobre la flora del departamento de Huánuco, mediante el análisis de los resultados de nuestra investigación más la recopilación de la información existente sobre este tema.

\section{MATERIAL Y MÉTODOS}

\section{Área de estudio}

La presente investigación se desarrolló en el departamento de Huánuco $\left(36850 \mathrm{~km}^{2}\right)$, ubicado en la parte central del Perú, aproximadamente entre los $8^{\circ} 26^{\prime} 22^{\prime \prime}$ y $10^{\circ} 28^{\prime} 29^{\prime \prime}$ de Latitud Sur y $74^{\circ} 22^{\prime} 20^{\prime \prime}$ y $77^{\circ} 19^{\prime} 16^{\prime}$ " de Longitud Oeste (Figura 1). Fisiografía. Es heterogénea, presenta dos provincias fisiográficas: una constituída por las superficies de topografía plana, denominada Llanura Amazónica, la cual está constituida por depósitos fluviales recientes y subrecientes que originan la zona de terrazas bajas inundables y no inundables; la otra provincia Colinosas y Montañosas constituída por las tierras altas y abruptas, conformada por la Cordillera Andina y la Cordillera Subandina, formada por materiales sedimentarios e ígneos (Escobedo, 2010). Clima. Presenta varios tipos de climas. Para el sector de la selva de Huánuco se ha identificado 7 tipos de climas, las cuales son: Clima súper-húmedo $\mathrm{y}$ cálido, Clima súper-húmedo $\mathrm{y}$ templado frío, Clima húmedo y semicálido, Clima ligeramente-húmedo y cálido, Clima moderadamente-húmedo y cálido, Clima moderadamente-húmedo y cálido, y Clima moderadamente-húmedo y semi-frío. La temperatura oscila entre de 22 y $32^{\circ} \mathrm{C}$, la precipitación media mensual mínima es de $55,1 \mathrm{~mm}$, y la máxima de $619,9 \mathrm{~mm}$. La humedad relativa media mensual varía entre $75 \%, 83 \%$ y $92 \%$ para el Llano Amazónico, Cordillera Subandina y Cordillera Oriental, respectivamente (Rodríguez, 2010). Vegetación. Presenta Bosque achaparrado (2800 - $3700 \mathrm{msnm})$, bosque montano (2300-2800 msnm) y bosque premontano $(800-2300 \mathrm{msnm})$ (Gonzáles, 2013). Mientras que Zárate \& Mori (2010) indican que la vegetación de la "Selva de Huánuco" se puede agrupar en tres grandes escenarios biofísicos. El primero es la Cordillera Oriental, el segundo es la Cordillera Subandina y el tercero es la Cuenca Amazónica. La vegetación de la Cordillera Oriental presenta al menos ocho formaciones vegetales, contiene los pajonales altoandinos, los arbustales de montañas altas, los bosques de montañas y otros, mientras que la cordillera subandina presenta comunidades vegetales boscosas, y en la vegetación amazónica encontramos bosques, palmerales y herbazales inundables y bosques no inundables.

\section{Unidades de muestreo en campo}

Ubicación de las unidades de muestreo. Se establecieron unidades de muestreo empleando imágenes satelitales LandSat (de $30 \mathrm{~m}$ de resolución) a fin de identificar los posibles tipos de vegetación existentes para el departamento de Huánuco. Establecimiento de las parcelas y trayectos. Se establecieron un total de 30 unidades de muestreo: 13 cuadrangulares (parcelas de $50 \times 20$ m o $50 \times 10 \mathrm{~m}$ ) y 17 trayectos de 10 a $80 \mathrm{~m}$ de largo, dispuestas en 22 tipos de vegetación (ver Tabla 1). Las parcelas grandes de $50 \times 20 \mathrm{~m}$, presentaron cuatro sub parcelas pequeñas de $5 \times 5 \mathrm{~m}$ situadas en las esquinas. Para las unidades de vegetación más diversas se emplearon parcelas y trayectos para las menos diversas. 

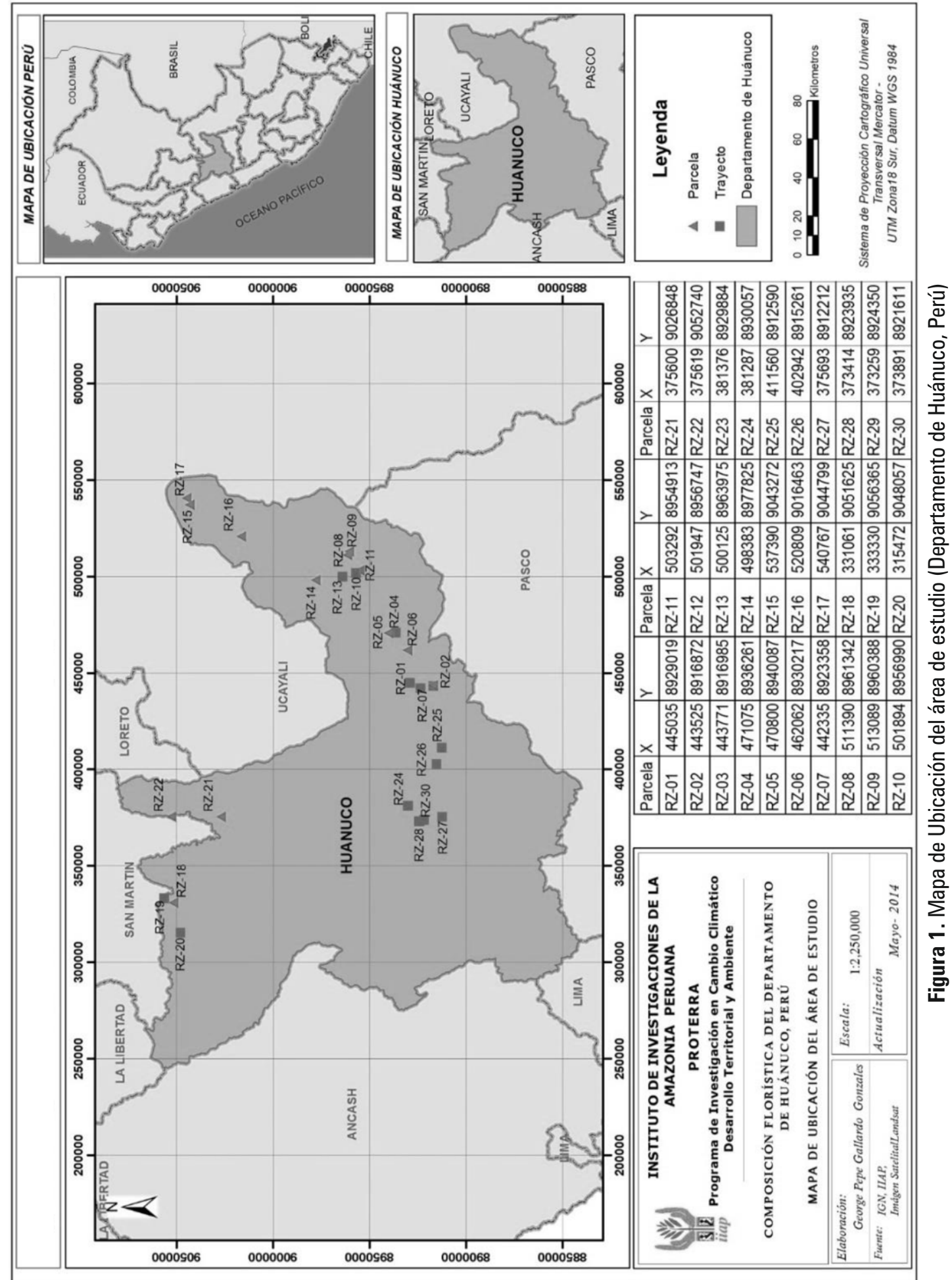
Tabla 1. Coordenadas geográficas de las unidades de muestreo en el Departamento de Huánuco

\begin{tabular}{|c|c|c|c|c|c|}
\hline $\mathbf{N}^{\mathbf{O}}$ & Parcela & $\begin{array}{l}\text { UTM } \\
\text { East }\end{array}$ & $\begin{array}{l}\text { UTM } \\
\text { North }\end{array}$ & $\begin{array}{l}\text { Altitud } \\
(\mathrm{m})\end{array}$ & Unidad de Muestreo \\
\hline 1 & RZ-01 & 445035 & 8929019 & 416 & Trayecto de $20 \mathrm{~m}$ \\
\hline 2 & RZ-02 & 443525 & 8916872 & 176 & Trayecto de $10 \mathrm{~m}$ \\
\hline 3 & RZ-03 & 443771 & 8916985 & 578 & Parcela de $50 \times 20 \mathrm{~m}$ \\
\hline 4 & RZ-04 & 471075 & 8936261 & 306 & Trayecto de $30 \mathrm{~m}$ \\
\hline 5 & RZ-05 & 470800 & 8940087 & 286 & Parcela de $50 \times 20 \mathrm{~m}$ \\
\hline 6 & RZ-06 & 462062 & 8930217 & 326 & Parcela de $50 \times 20 \mathrm{~m}$ \\
\hline 7 & RZ-07 & 442335 & 8923358 & 758 & Trayecto de $10 \mathrm{~m}$ \\
\hline 8 & RZ-08 & 511390 & 8961342 & 303 & Parcela de $50 \times 20 \mathrm{~m}$ \\
\hline 9 & RZ-09 & 513089 & 8960388 & 399 & Parcela de $50 \times 20 \mathrm{~m}$ \\
\hline 10 & RZ-10 & 501894 & 8956990 & 221 & Trayecto de $20 \mathrm{~m}$ \\
\hline 11 & $R Z-11$ & 503292 & 8954913 & 227 & Parcela de $50 \times 20 \mathrm{~m}$ \\
\hline 12 & RZ-12 & 501947 & 8956747 & 201 & Trayecto de $10 \mathrm{~m}$ \\
\hline 13 & RZ-13 & 500125 & 8963975 & 197 & Trayecto de $20 \mathrm{~m}$ \\
\hline 14 & RZ-14 & 498383 & 8977825 & 257 & Parcela de $50 \times 20 \mathrm{~m}$ \\
\hline 15 & RZ-15 & 537390 & 9043272 & 164 & Parcela de $50 \times 20 \mathrm{~m}$ \\
\hline 16 & RZ-16 & 520809 & 9016463 & 231 & Parcela de $50 \times 10 \mathrm{~m}$ \\
\hline 17 & RZ-17 & 540767 & 9044799 & 159 & Parcela de $50 \times 10 \mathrm{~m}$ \\
\hline 18 & RZ-18 & 331061 & 9051625 & 875 & Parcela de $50 \times 10 \mathrm{~m}$ \\
\hline 19 & RZ-19 & 333330 & 9056365 & 722 & Trayecto de $10 \mathrm{~m}$ \\
\hline 20 & RZ-20 & 315472 & 9048057 & 1480 & Trayecto de $20 \mathrm{~m}$ \\
\hline 21 & RZ-21 & 375600 & 9026848 & 558 & Parcela de $50 \times 10 \mathrm{~m}$ \\
\hline 22 & RZ-22 & 375619 & 9052740 & 728 & Parcela de $50 \times 20 \mathrm{~m}$ \\
\hline 23 & RZ-23 & 381376 & 8929884 & 2369 & Trayecto de $50 \mathrm{~m}$ \\
\hline 24 & RZ-24 & 381287 & 8930057 & 2231 & Trayecto de $30 \mathrm{~m}$ \\
\hline 25 & RZ-25 & 411560 & 8912590 & 2032 & Trayecto de $80 \mathrm{~m}$ \\
\hline 26 & RZ-26 & 402942 & 8915261 & 2912 & Trayecto de $30 \mathrm{~m}$ \\
\hline 27 & RZ-27 & 375693 & 8912212 & 1830 & Trayecto de $70 \mathrm{~m}$ \\
\hline 28 & RZ-28 & 373414 & 8923935 & 3521 & Trayecto de $50 \mathrm{~m}$ \\
\hline 29 & RZ-29 & 373259 & 8924350 & 3520 & Trayecto de $30 \mathrm{~m}$ \\
\hline 30 & RZ-30 & 373891 & 8921611 & 3476 & Trayecto de $10 \mathrm{~m}$ \\
\hline
\end{tabular}




\section{Organismos muestreados}

En la evaluación se incluyeron todas las Angiospermas, Gimnospermas, Pteridofitas y Licofitas. Para la unidades mayores (parcela de $50 \mathrm{x}$ $20 \mathrm{~m}$ y de $50 \times 10 \mathrm{~m}$ ) se consideraron los individuos con fustes mayores o iguales a $10 \mathrm{~cm}$ de DAP (diámetro a la altura del pecho), lo cual incluyen árboles, arbustos, arborescentes, palmeras y bejucos, mientras que para las subunidades menores $(5 \times 5 \mathrm{~m})$ se tomaron todos aquellos individuos menores o iguales a $1 \mathrm{~cm}$ de DAP, las cuales incluyen, arbolitos, arbustos, enredaderas y hierbas. Para los muestreos en trayectos se consideraron todas las formas de vida presentes.

\section{Herborización}

La herborización (colecta, prensado, empacado, preservación y secado) de las muestras fue realizada siguiendo lo propuesto por Judd et al., (1999). Para la identificación de los especímenes se empleó bibliografía especializada (Spichiger et al., 1989; Berg et al., 1990; Pennington, 1990; Gentry, 1993; Henderson, 1995; Vásquez, 1997; Esser, 1999; Ribeiro et al., 1999; Prance, 2001; Pennington et al., 2004; Amasifuen \& Zárate, 2005; Berg, 2005); y se comparó con las exicatas de los Herbarios USM y AMAZ. Se actualizó los estados taxonómicos de las especies comparándolos con las base de datos en línea de The Plant List (http://www.theplantlist.org). El sistema de clasificación de las familias empleado en el proceso taxonómico fue el propuesto por Christenhusz et al. (2011b) para las Licofitas, Smith et al. (2006) para las Pteridofitas, Christenhusz et al. (2011a) para las Gimnospermas y APG III (2009) para las Angiospermas. Para facilitar el proceso de elaboración de la base de datos se ha utilizado la herramienta de "Taxonomic Name Resolution Service v3.2" (http://tnrs.iplantcollaborative.org/ TNRSapp.html) para las Angiospermas. Los estados taxonómicos de las especies recopiladas en la bibliográfia también se trataron de acuerdo al procedimiento mencionado anteriomente. Posteriormente las muestras fueron depositadas en el Herbario Herrerense del IIAP.

\section{Fuentes de información}

Las referencias biliográficas utilizadas con presencia de un alto contenido de especies que habitan en Huánuco son: Brako \& Zaruchi (1993), Arévalo (1998),Ulloa et al. (2004), Salinas (2005), León et al. (2006), Rodríguez et al. (2006), Salvador et al. (2006), Moreno et al. (2007), Vidal \& Ramos (2008), Cachique (2009), Salvador et al. (2009), Castillo (2009), Gonzáles (2012), García (2013).
También se utilizó Uriol (1980), Albitres (1981), Vega (1986), Cervantes (1994), Zacarias (2001), Huayanay (2002), Facho (2004), Bustamante (2005), Lambruschini (2005), Salazar (2005), Gonzáles (2006), Simón (2006), Bernardo (2007), Oré (2007), Paucar (2007), Pulido (2007), Encarnación (2009), Pinchi (2009), Cabrera (2010), Capillo (2010), Coz (2010), Pozo (2010), Salazar (2010), Viera (2010), Chavarría (2011), Espinoza (2011), Falcón (2011), Herrera (2011), Pedroso (2011), Sánchez (2011), Valdez (2011), Vargas (2011), Vega (2011), Espinoza (2012), Ulda (2012) y Ore (2013) las cuales contienen información sobre la presencia de al menos una especie en el departamento.

\section{RESULTADOS Y DISCUSIÓN}

En el presente trabajo se registró 1423 individuos de plantas (Licofitas, Pteridofitas, Gimnospermas y Angiospermas), que corresponde a 756 especies (354 especies y 402 morfoespecies), 403 géneros y 130 familias. Las familias más abundantes fueron: Arecaceae (147 ind., 10.4\%), Fabaceae (115 ind., $8.1 \%)$, Euphorbiaceae (95 ind., 6.7\%), Myristicaceae (50 ind., 3.5\%), Moraceae (48 ind., $3.4 \%$ ), Asteraceae (46 ind., 3.2\%), Rubiaceae (43 ind., $3 \%$ ) y otras. Las familias con la mayor cantidad de especies fueron: Fabaceae (60 esp., 7.9\%), Euphorbiaceae (32 esp., 4.2\%), Rubiaceae (29 esp., $3.8 \%$ ), Asteraceae (27 esp., 3.6\%), Moraceae (26 esp., 3.4\%), Araceae (25 esp., 3.3\%), Annonaceae (23 esp., 3\%), Melastomataceae (23 esp., 3\%), Piperaceae (22 esp., 2.9\%), Sapotaceae (17 esp., 2.2\%), Meliaceae (16 esp., 2.1\%), Lauraceae (15 esp., 2\%), entre otras. Las especies con mayor cantidad de individuos fueron: Mauritia flexuosa (91 ind., 6.4\%), Socratea exorrhiza (21 ind., 1.5\%), Virola pavonis (20 ind., 1.4\%), Matisia cordata (19 ind., 1.3\%), Inga aff. ingoides (15 ind., 1.1\%), Drypetes amazonica (14 ind., 1\%), Euterpe precatoria (12 ind., $0.8 \%$ ), Nealchornea yapurensis (12 ind., 0.8\%), Pseudolmedia macrophylla (11 ind., $0.8 \%$ ), Rinorea flavescens (10 ind., 0.7\%), Bauhinia longicuspis (9 ind., 0.6\%), Otoba parvifolia (9 ind., $0.6 \%$ ), entre otras.

En 1993 Brako \& Zaruchi reportaron para el Departamento de Huánuco 4287 especies de Angiospermas y Gimnospermas. Luego en el 2004 Ulloa et al. publicó para Huánuco 131 especies más, y en el 2006 Rodríguez et al. añadieron 9 especies más. Adicionalmente hay varias publicaciones en las que se indican listas de especies para el departamento como: Arévalo (1998), Salinas (2005), Moreno et al. (2007), Vidal \& Ramos (2008) 
Cachique (2009), Castillo (2009), Salvador et al. (2009), Gonzáles (2012), García (2013) y otros. En el presente reporte encontramos 352 especies de plantas con nombres latinos, de las cuales 151 no estaban reportadas anteriormente para Huánuco.

Entonces uniendo la información disponible (detallado en Métodos), actualmente para el departamento de Huánuco se reportan un total de 4712 especies incluidas en 226 familias y 1443 géneros de plantas (Licofitas (7 especies), Pterydofitas (250 especies), Gimnospermas (7 especies) y Amgiospermas (4453 especies)). La lista de especies se presenta en Anexo. ${ }^{1}$. Adicionalmente existen 39 nombres científicos de plantas que se encuentran en el estado taxonómico incierto ya que Taxonomic Name Resolution Service v3.2 no asigno el estado de nombre "aceptado" o "sinónimo", por tal motivo no fueron consideradas en el análisis; estos nombres son: Polyalthia cinnamomea, Senecio repens, Fridericia pilulifera, Guzmania calothyrsus, Stigmaphyllon puberum, Miconia theizans, Benzingia caudate, Lepanthes pastorellii, Lepanthes pedunculata, Lepanthes sijmii, Lepanthes tricuspidata, Lepanthes vulpina, Masdevallia manningii, Sobralia ruparupaensis, Stelis hutchisonii, Trichopilia mesoperuviensis, Vitekorchis aurifera, Warczewiczella amazonica, Peperomia puberulibacca, Erythroxylum acuminatum, Acacia polyphylla, Caesalpinia spinosa, Senna galegifolia, Heliconia ortotricha, Struthanthus acuminatus, Parodiolyra micrantha, Hesperomeles heterophylla, Hesperomeles lanuginosa, Smilax purhampuy, Smilax ruiziana, Cestrum strigillatum, Solanum bukasovii, Hymenophyllum lamellatum, Axinaea dependens, Solanum quercifolium, Neodryas alba, Trichipteris flava, Diplazium flexuosum y Diplazium paucijugum. Estos nombres inciertos indican la falta de mayores estudios taxonómicos en esos grupos de plantas.

Las 40 especies más abundantes fueron (de acuerdo a su presencia en cada publicación, organizados de mayor a menor cantidad de individuos): Caesalpinia spinosa (Molina) Kuntze, Calycophyllum spruceanum (Benth.) Hook.f. ex K.Schum., Adiantum obliquum Willd., Bartsia inaequalis Benth., Besleria gracilenta C. Morton, Calceolaria inflexa Ruiz \& Pav., Calceolaria pavonii Benth., Calliandra taxifolia (Kunth) Benth., Carica papaya L., Cavendishia punctata (Ruiz \& Pav. ex J.St.Hil.) Sleumer, Cinchona glandulifera Ruiz \& Pav., Cinchona micrantha Ruiz \& Pav., Cischweinfia parva (C.Schweinf.) Dressler \& N.H.Williams, Clavija macrocarpa Ruiz \& Pav.,
Condaminea corymbosa (Ruiz \& Pav.) DC., Cyrtochilum cimiciferum (Rchb.f.) Dalström, Dalea cylindrica Hooker., Distichia muscoides Nees \& Meyen, Dodonaea viscosa (L.) Jacq., Epidendrum compressibulbum D.E.Benn. \& Christenson, Miconia splendens (Sw.) Griseb., Mimulus glabratus Kunth, Monnina marginata C. Presl, Nephrolepis cordifolia (L.) C. Presl, Oxalis tuberosa Molina, Peperomia serpens (Sw.) Loudon, Phaseolus vulgaris L., Pilea dombeyana Wedd., Pityrogramma calomelanos (L.) Link, Plantago australis Lam., Plantago sericea Ruiz \& Pav., Plantago tubulosa Decne., Pteris altissima Poir., Seemannia sylvatica (Kunth) Baill., Senecio comosus Sch.Bip., Solanum sessiliflorum Dunal, Solanum tuberosum L., Spartium junceum L., Theobroma cacao L. y Tropaeolum tuberosum Ruiz \& Pav. Las 10 familias con las mayores cantidades de individuos fueron: Orchidaceae (395 ind., 7.12\%), Asteraceae (324 ind., 5.84\%), Melastomataceae (299 ind., 5.39\%), Fabaceae (267 ind., 4.81\%), Rubiaceae (246 ind., 4.43\%), Poaceae (236 ind., 4.25\%), Piperaceae (225 ind., 4.06\%), Solanaceae (123 ind., 2.22), Araceae (120 ind., $2.16 \%$ ) y Bromeliaceae (101 ind., $1.82 \%$ ). Y las familias con la mayor riqueza fueron: Orchidaceae (331 esp., 7.02\%), Asteraceae (305 esp., 6.47\%), Melastomataceae (237 esp., 5.02\%), Fabaceae (221 esp., 4.69\%), Poaceae (216 esp., 4.58\%), Piperaceae (215 esp., 4.56\%), Rubiaceae (197 esp., 4.18\%), Solanaceae (113 esp., 2.4\%), Araceae (98 esp., $2.08 \%$ ), Acanthaceae (93 esp., 1.97\%) y otras.

El departamento de Huánuco con 4712 especies de plantas esta ubicado en el segundo lugar después de Loreto con 5112 especies (Brako \& Zaruchi, 1993) y antes de Junín, Cuzco y San Martín. Esta alta diversidad de plantas en el departamento pobablemente esta relacionado a su alta variedad climática (Rodríguez, 2010) y fisiográfica (Escobedo, 2010).

\section{AGRADECIMIENTOS}

Al AMAZ, USM, Servicios de biodiversidad, Marcos Ríos y Hamilton Beltran por su colaboración en la identificación botánica. A Dillys M. Vela, Gianina J. Mundo y Eber E. Flores por sus contribuciones científicas. A Wilder Macedo por los equipos proporcionados. A Jaime Reátegui por colaborar con nosotros en la fase de campo. A Ediberto Chuculin, Warren Ríos, Manuel Nique, Yula Ruíz, Hugo Huapalla, Juan Huapalla y Hector Huapalla por la información bibliográfica proporcionada. ¡Muchas gracias!

1. http://folia.iiap.org.pe/index.php/foliaamazonica/index 


\section{BIBLIOGRAFÍA CITADA}

Albitres, L. 1981. Influencia de la materia orgánica $y$ del fósforo en el desarrollo de plantas de vivero de cacao (Theobroma cacao) bajo condiciones de tingo maría. Tesis de pre-grado. Universidad Nacional Agraría de la Selva. Tingo María, Perú. $114 \mathrm{pp}$.

Amasifuen, C.; Zárate, R. 2005. Composición Taxonómica, Ecología y Periodo de Floración de Plantas Leñosas "Dicotiledóneas". Tesis de pre-grado. Universidad Nacional de la Amazonía Peruana. Iquitos-Perú. 397pp.

APG III. 2009. An update of the Angiosperm Phylogeny Group classification for the orders and families of flowering plants: APG III. Botanical Journal of the Linnean Society, 141 (4): 399-346.

Arévalo, C. 1998. Identificación y usos de los Helechos (Clase: Filicopsida) en la zona de Tingo María (Huánuco, Perú). Tesis de pregrado. Universidad Nacional Agraría de la Selva. Tingo María, Perú. 196pp.

Berg, C.; Akkermans, R.; Van Heusden, H. 1990. Cecropiaceae: Coussapoa and pourouma, with an introducction to the Family. Flora Neotropica. Monograph 51.208.

Berg, C.; Franco, P. 2005. Cecropia. Flora Neotropica. Monograph 94. 1a. ed. 230.

Bernardo, W. 2007. Introducción de 25 líneas de cebada (Hordeum vulgare L.) en el Valle del Nupe. Tesis de pre-grado. Universidad Nacional Hermilio Valdizán. Huánuco, Perú. 79pp.

Brako, L.; J. Zarucchi. 1993. Catalogue of the flowering plants and gymnosperms of Peru. Monogr. Syst. Bot. Missouri Bot. Gard. 45: 1286 p.

Bustamante, H. 2005. Efecto de la densidad de siembra en el rendimiento del cultivo de brócoli (Brassica oleracea L. Italica) en condiciones agroecológicas del Valle de Huánuco 2004. Tesis de pre-grado. Universidad Nacional Hermilio Valdizán. Huánuco, Perú. 54pp.

Cabrera, T. 2010. Evaluación agronómica y fenológica de morfotipos de Oca (Oxalis tuberosa Mol), en la comunidad de Huayllacayán-Huánuco. Tesis de pre-grado. Universidad Nacional Hermilio Valdizán. Huánuco, Perú. 99pp.

Cachique, E. 2009. Diversidad de las plantas epifitas vasculares en especies arbóreas del Jardin botánico de la Universidad Nacional Agraria de la Selva. Tesis de pre-grado. Universidad Nacional Agraría de la Selva. Tingo María, Perú. 92pp.
Capillo, P. 2010. Efecto del biocida sumo de Cabuya Agave americana L. en el control de la pulguilla Epitrix spp. Del cultivo de papa Solanum tuberosum L. variedad Canchán en condiciones agroecológicas de Huacrachuco-2009. Tesis de pre-grado. Universidad Nacional Hermilio Valdizán. Huánuco, Perú. 85pp.

Castillo, M. 2009. Estudio dendrológico de la familia Rubiaceae en la zona de Tingo María, Perú. Tesis de pre-grado. Universidad Nacional Agraría de la Selva. Tingo María, Perú. 148pp.

Cervantes, M. 1994. Efecto de diferentes números de plantas por golpe y distanciamiento de siembra en el rendimiento de dos variedades de arroz (Oryza sativa L.) bajo riego en Camaná. Tesis de pre-grado. Universidad Nacional Agraría de la Selva. Tingo María, Perú. 107pp.

Chavarría, A. 2011. Efecto de la fertiliozación inorgánica en el rendimeinto del cultivo de cebada (Hordeum vulgare L.) variedad INIA 416 La Milagrosa en condiciones agroecológicas de Huacrachuco, 2010. Tesis de pre-grado. Universidad Nacional Hermilio Valdizán. Huánuco, Perú. 81pp.

Christenhusz, M.; Reveal, J.; Farjon, A.; Gardner, M.; Mill, R.; Chase, M. 2011a. A new classification and linear sequence of extant gymnosperms. Phytotaxa, 19: 55-70.

Christenhusz, M.; Zhang, X.; Schneider, H. 2011b. A linear sequence of extant families and genera of lycophytes and ferns. Phytotaxa, 19:7-54.

Coz, Y. 2010. Caracterización in situ de duraznos criollos (Prunus persica (L.) Batsch) potencialmente comerciales en cinco localidades del distrito de Churubamba Huánuco. Tesis de pre-grado. Universidad Nacional Hermilio Valdizán. Huánuco, Perú. 96pp.

Encarnación, Z. 2009. Efecto de productos biológicos y químicos en el cultivo de papa nativa (Solanum goniocalyx cv. Iscu phuru) en tres condiciones altitudinales de la comunidad campesina Huayllacayan, Kichki - Huánuco. Tesis de pre-grado. Universidad Nacional Hermilio Valdizán. Huánuco, Perú.

Escobedo, R. 2010. Fisiografía, informe temático. Proyecto Mesozonificación Ecológica y Económica para el Desarrollo Sostenible de la selva de Huánuco. Convenio entre el IIAP, DEVIDA. Iquitos - Perú.

Espinoza, A. 2012. Efecto de diferentes dosis de gaicashi, en el crecimiento inicial de plantas de pino chuncho (Schizolobium amazonicum Huber ex Ducke) producidae en tubetes. Tesis de pregrado. Universidad Nacional Agraría de la Selva. Tingo María, Perú. 66pp. 
Espinoza, J. 2011. La densidad de siembra en el rendimiento del cultivo de frijol (Phaseolus vulgaris L.) variedad Canario 2000 en condiciones agroecológicas de la localidad de Hиánuco Huacrachuco - 2009. Tesis de pregrado. Universidad Nacional Hermilio Valdizán. Huánuco, Perú. 73pp.

Esser, H. 1999. Rhodothyrsus, a new genus of Euphorbiaceae from tropical South America. Brittonia, 51 (2): 170-180.

Facho, M. 2004. Estudio fenológico y de rendimiento de dos cultivares: PTU-405 y PTM331 de papayo (Carica papaya L.) en Tulumayo. Tesis de pre-grado. Universidad Nacional Agraría de la Selva. Tingo María, Perú. 102pp.

Falcón, G. 2011. Manejo fisionutricional del cultivo de zapallo (Cucurbita maxima), en el Valle de Higueras Huánuco. Tesis de pre-grado. Universidad Nacional Hermilio Valdizán. Huánuco, Perú. 90pp.

Font, P. 1985. Diccionario de Botánica. Ed. LABOR, S. A. Madrid, España. 1244pp.

García, E. 2013. Dendrología de la familia Melastomataceae de los bosques secundarios de la Provincia Lencio Prado, Huánuco. Tesis de pre-grado. Universidad Nacional Agraría de la Selva. Tingo María, Perú. 103pp.

Gentry, A. 1993. A Field Guide to the Families and Genera of Woody Plants of Northwest South America (Colombia, Ecuador, Perú) with supplementary notes on herbaceous taxa. Conservation International. Washington-USA. 895pp.

Gonzáles M. 2013. Ecología y Conservación de aves en el Bosque Montano de Huánuco. Informe Final. Grupo Aves del Perú. Universidad Nacional Agraria de la Selva - Tingo María. $107 \mathrm{pp}$.

Gonzáles, R. 2006. Efecto de dosis del biol en variedades de camote (Ipomoea batata L.) en el valle del Huallaga - Huánuco. Tesis de pregrado. Universidad Nacional Hermilio Valdizán. Huánuco, Perú.

Gonzáles, R. 2012. "Estudio Taxonomicoo de las Plantagináceas en los Andes Centrales (Ancash, Lima Huanuco, Pasco y Junín) del Perú". Tesis de pre-grado. Universidad Nacional Mayor de San Marcos. 84pp.

Henderson, A. 1995. The Palms of the Amazon. Oxford University Press, Inc. New York, USA. 362pp.

Herrera, R. 2011. La fertilización orgánica e inorgánica en el rendimiento del trigo (Triticum aestivum - var. Centenario) en condiciones agroecológicas de Huacrachuco , Нuánuco 2009. Tesis de pre-grado. Universidad Nacional Hermilio Valdizán. Huánuco, Perú. 85pp.

Huayanay, H. 2002. Evaluación de la calidad de 8 ecotipos de cocona (Solanum toripo HBK.). Universidad Nacional Agraria de la Selva Tingo María. 85pp.

Judd, W., Campbell, C., Kellogg, E.; Stevens, P. 1999. Plant Systematics A phylogenetic approach. Sinauer Associates, Inc. Sunderland, Reino Unido. 464pp.

Lambruschini, I. 2005. Formas de propagación del yacon (Smallanthus sonchifolius) en el valle de Huánuco. Tesis de pre-grado. Universidad Nacional Hermilio Valdizán. Huánuco, Perú. $55 \mathrm{pp}$.

León, B.; Roque, J.; Ulloa, C.; Pitman, N.; Jorgensen, P.; Cano, A. 2006. El libro rojo de las plantas endémicas del Perú. Revista Peruana de Biología, 13(2). 971.

Moreno, C. 2007. Fabaceae potencialmente útiles de la provincia de Huánuco. Rev. Investigación Valdizana, 1 (1): 30-34.

Oré, L. 2007. Evaluación del tratamiento térmico en conserva mixta de papaya (Carica papaya L.) y cocona (Solanum toripo HBK.) en envases de hojalata. Universidad Nacional Agraría de la Selva. Tingo María, Perú. 153pp.

Ore, R. 2013. Comportamiento de plantas de bolaina blanca (Guazuma crinita C. Mart) a diferentes edades de terreno definitivo, Tingo María. Tesis de pre-grado. Universidad Nacional Agraría de la Selva. Tingo María, Perú. 53pp.

Paucar, M. 2007. Caracterización morfológica y etnobotánica de ocas (Oxalis tuberosa Mol) cultivadas in situ en las comunidades del distrito de Kichki - Huánuco. Tesis de pre-grado. Universidad Nacional Hermilio Valdizán. Huánuco, Perú. 118pp.

Pedroso, R. 2011. Efecto de la densidad de siembra en el rendimiento de grano verde del cultivo de arveja (Pisum sativum L.) variedad INIA 103 remate en condiciones agroecológicas de Gochachilca Huacrachuco - 2010. Tesis de pregrado. Universidad Nacional Hermilio Valdizán. Huánuco, Perú. 65pp.

Pennington, T. 1990. Sapotaceae. Flore Neotrópica. Monograph 52.770p.

Pennington, T., Reynel, C. y Daza, A. 2004. Illustred guide to the Trees of Peru. First published. England- United Kingdom. David Hunt, The manse, Chapel Lane, Milborne Port Sherborne, DT9DL. 847pp. 
Pinchi, H. 2009. Efecto de diferentes dosis de Bocashi em, sobre crecimiento en vivero de plantas de castaña (Bertholletia excelsa HBK.), producidas en tubetes. Tesis de pre-grado. Universidad Nacional Agraría de la Selva. Tingo María, Perú. 46pp.

Pozo, J. 2010. Evaluación del efecto de humus en el rendimiento de los cultivares de Haba (Vicia faba L.), en condiciones agro ecológicas de la zona andina de Margos-Huánuco. Tesis de pregrado. Universidad Nacional Hermilio Valdizán. Huánuco, Perú. 101pp.

Prance, G. 2001. Chrysobalanaceae. Flora de Colombia. Monografía $\mathrm{N}^{\circ}$ 19. 1a.ed. BogotaColombia. UNIBIBLOS. 292pp.

Pulido, H. 2007. Caracterización morfológica y etnobotánica de Mashua (Tropaeolum tuberosum Ruiz y Pav.) cultivadas in situ en el distrito de Kichki-Huánuco. Tesis de pre-grado. Universidad Nacional Hermilio Valdizán. Huánuco, Perú. 118pp.

Ribeiro, J., Hopkins, M., Vicentini, A., Sothers, C., Costa, M., Brito, J., Souza, M., Martins, L., Lohmann, L., Assuncao, P., Pereira, E., Silva, C., Mesquita, M.; Procópio, L. 1999. Flora da Reserva Ducke.. Guia de identificaçao das plantas vasculares de uma floresta de terra-firme na Amazônia Central. 1a. ed. Manaus-Brasil. INPA. Midas Printing. 799pp.

Rodríguez, E.; Vásquez, R.; Rojas, R.; Calatayud, G.; León, B.; Campos, J. 2006. Nuevas adiciones de angiospermas a la flora del Perú Angiosperms additions to flora of Peru. Revista Peruana de Biología, 13(1):129-138.

Rodríguez, E. 2010. Clima, informe temático. Proyecto Mesozonificación Ecológica y Económica para el Desarrollo Sostenible de la selva de Huánuco. Convenio entre el IIAP, DEVIDA. Iquitos - Perú. 36pp.

Salazar, L. 2010. Caracterización morfológica de Mashuas (Tropaeolum tuberosum $R$. \& P.) cultivadas ex situ en condiciones agroecológicas de la localidad de San Fernando-Huacrachuco 2009. Tesis de pre-grado. Universidad Nacional Hermilio Valdizán. Huánuco, Perú. 83pp.

Salazar, S. 2005. Efecto de materia orgánica en el rendimiento de dos variedades de alcachofa (Cynara scolymus L.) en el valle del Nupe. Tesis de pre-grado. Universidad Nacional Hermilio Valdizán. Huánuco, Perú. 87pp.

Salinas, I. 2005. "Estudio taxonomico del orden Scrophulariales (Magnoliopsida) en los bosques Montanos Húmedos de Carpish (Dpto. Huánuco, Perú)". Tesis de pre-grado.
Universidad Nacional Mayor de San Marcos. Lima, Perú. 150pp.

Sánchez, M. 2011. Efecto del abonamiento orgánico en el rendimiento de forraje RYE GRASS (Lolium multiflorum Lam.) en condiciones agroecológicas de Huayllacayán Huánuco 2010. Tesis de pre-grado. Universidad Nacional Hermilio Valdizán. Huánuco, Perú.

Salvador, F.; Alonso, M.; Ríos, S. 2006. "Adiciones a la flora andina peruana del departamento de Huánuco I". Candollea, 61 (2): 279-291.

Salvador, F.; Alonso, M.; Ríos, S. 2009. Tres nuevos registros del genero Carex (Cyperaceae) para el Perú y adiciones a la flora andina del departamento Huanuco". Revista Peruana de Biología, 15(2): 083-092.

Shimwell, D. 1971. Description \& Classificaction of Vegetation. Sidgwick \& Jackson Londres, Reino Unido. 322pp.

Simón, M. 2006. Efecto de los abonos orgánicos en el rendimiento de cultivares de claveles (Dianthus caryophillus L.), en condiciones agroecológicas de Chinchobamba Tomayquichua. Tesis de pre-grado. Universidad Nacional Hermilio Valdizán. Huánuco, Perú.

Smith, A.; Pryer, K.; Schuettpelz, E.; Korall. P.; Schneider, H.; Wolf, P. 2006. A classification for extant ferns. Taxon, 55 (3): 705-731.

Spichiger, R.; Méroz, J., Loizeau, P.; Stutz, L., 1989. Contribución a la Flora de la Amazonía Peruana: Los Árboles del Arboretum Jenaro Herrera. Vol.I 359pp. y Vol. II. 565pp.

Ulda, F. 2012. Efecto de la fertilización inorgánica en el rendimiento del cultivo de maíz (Zea mays L.) variedad Negra Tomasa, en condiciones agroecológicas de la localidad de Huacrachuco - 2010. Tesis de pre-grado. Universidad Nacional Hermilio Valdizán. Huánuco, Perú. $62 \mathrm{pp}$.

Ulloa, C.; Zarucchi, J.; León, B. 2004. Diez años de Adiciones a la Flora del Perú: 1993-2003. Arnaldoa (Edic. Esp. Noviembre 2004): 1-242.

Uriol, C. 1980. Sistemas de siembra por poblaciones de plantas en soya Glycine max L. Merril, variedad júpiter en Tulumayo. Tesis de pregrado. Universidad Nacional Agraría de la Selva. Tingo María, Perú. 71pp.

Valdez, E. 2011. Efectos de la fertilización orgánica e inorgánica en el rendimeinto del cultivo de avena forrajera (Avena sativa L.) variedad "INIA 901 - Mantaro 15 M" en condiciones agroecológicas de Quillabamba, Marañón. Tesis de pre-grado. Universidad Nacional Hermilio Valdizán. Huánuco, Perú. 
Vargas, D. 2011. Efecto de diferentes tipos de sustratos en el crecimiento de plantas de Capirona (Calycophyllum spruceanum (Bentham Hooker f. ex Schumann)) en fase de vivero. Tesis de pre-grado. Universidad Nacional Agraría de la Selva. Tingo María, Perú. 70pp.

Vásquez, R. 1997. Flórula de las Reservas Biológicas de Iquitos, Perú. 1a. ed. Missouri Botanical Garden Press. St. Louis, USA. 1046pp.

Vega, E. 1986. Efecto del uso de cuatro especies de plantas incorporadas en la producción de arroz (Oryza sativa L.) en el sistema de secano. Tesis de pre-grado. Universidad Nacional Agraría de la Selva. Tingo María, Perú. 87pp.

Vega, G. 2011. Efecto de la fertilización y aplicación de materia orgánica en el rendimiento del cultivo de papa, variedad canchan en el Distritio de Baños - 2009. Tesis de pre-grado. Universidad Nacional Hermilio Valdizán. Huánuco, Perú. $68 \mathrm{pp}$.

Vidal, R.; Ramos, F. 2008. "Conocimiento y Utilización de los Recursos Naturales para estimular el Ecoturismo en la microcuenca de Lanjas, Huanuco. Investigación Valdizana, 2 (2): 92-95.

Viera, S. 2010. Caracterización agromorfológica ex situ de accesiones de Olluco (Ullucus tuberosus Caldas) en condiciones agroecológicas de san Fernando Huacrachuco-2009. Tesis de pregrado. Universidad Nacional Hermilio Valdizán. Huánuco, Perú. 79pp.

Zacarias, A. 2001. Evaluación de la compatibilidad de injertación entre Coffea canephora P. y Coffea arabica L. y su crecimiento con uno y dos plantas por bolsa en vivero. Tesis de pre-grado. Universidad Nacional Agraría de la Selva. Tingo María, Perú. 90pp.

Zárate, R.; Mori, T. 2010. Vegetación, informe temático. Proyecto Mesozonificación Ecológica y Económica para el Desarrollo Sostenible de la selva de Huánuco. Convenio entre el IIAP, DEVIDA. Iquitos-Perú.

Recibido: 10 de marzo del 2015

Aceptado para publicación: 28 de mayo del 2015 
Tabla 2. Lista de especies de Angiospermas, Gimnospermas, Pteridofitas y Licofitas del Departamento de Huánuco, Perú.

\begin{tabular}{|l|}
\hline Acanthaceae \\
\hline Aphelandra acrensis Lindau \\
\hline Aphelandra aurantiaca (Scheidw.) Lindl. \\
\hline Aphelandra campii Wassh. \\
\hline Aphelandra cirsioides Lindau \\
\hline Aphelandra ferreyrae Wassh. \\
\hline Aphelandra formosa (Bonpl.) Nees \\
\hline Aphelandra glabrata Willd. ex Nees \\
\hline Aphelandra jacobinioides Lindau \\
\hline Aphelandra latibracteata Wassh. \\
\hline Aphelandra maculata (Tafalla ex Nees) Voss \\
\hline Aphelandra mucronata (Ruiz \& Pav.) Nees \\
\hline Aphelandra pulcherrima (Jacq.) Kunth \\
\hline Aphelandra rosulata (Lindau) Wassh. \\
\hline Aphelandra weberbaueri Mildbr. \\
\hline Dicliptera peruviana (Lam.) Juss. \\
\hline Dicliptera ruiziana Wassh. \\
\hline Dyschoriste ciliata (Nees) Kuntze \\
\hline Fittonia albivenis (Lindl. ex Veitch) Brummitt \\
\hline Fittonia gigantea Linden ex André \\
\hline Hemigraphis alternata (Burm. f.) T. Anderson \\
\hline Hygrophila costata Nees \& T. Nees \\
\hline Justicia aequilabris (Nees) Lindau \\
\hline Justicia appendiculata (Ruiz \& Pav.) Vahl \\
\hline Justicia comata (L.) Lam. \\
\hline Justicia iochila Mildbr. \\
\hline Justicia lancifolia (Nees) V.M. Badillo \\
\hline Justicia lineolata Ruiz \& Pav. \\
\hline Justicia pectoralis Jacq. \\
\hline Justicia pilosa (Ruiz ex Nees) Lindau \\
\hline Justicia poeppigiana (Nees) Lindau \\
\hline Justicia pozuzoensis Wassh. \\
\hline Justicia racemosa Ruiz \& Pav. \\
\hline Justicia ruiziana Lindau \\
\hline Justicia secundiflora (Ruiz \& Pav.) Vahl \\
\hline Justicia sericea Ruiz \& Pav. \\
\hline Justicia soukupii (Standl. \& F.A. Barkley) V.A.W. Graham \\
\hline Justicia tenuiflora Ruiz \& Pav. \\
\hline Justicia tenuistachys (Rusby) Wassh. \& J.R.I. Wood \\
\hline Justicia ulei Lindau \\
\hline Justicia yurimaguensis Lindau \\
\hline Kalbreyeriella gigas Leonard \\
\hline Mendoncia aspera Ruiz \& Pav. \\
\hline Mendoncia glabra Poepp. \& Endl. \\
\hline Mendoncia hoffmannseggiana Nees \\
\hline
\end{tabular}

\section{Lythraceae}

Adenaria floribunda Kunth

Ammannia latifolia L.

Cuphea carthagenensis (Jacq.) J.F. Macbr.

Cuphea ciliata Ruiz \& Pav.

Cuphea cordata Ruiz \& Pav.

Lafoensia acuminata (Ruiz \& Pav.) DC.

\section{Malpighiaceae}

Banisteriopsis muricata (Cav.) Cuatrec.

Banisteriopsis padifolia (Poepp. ex Nied.) B. Gates

Bronwenia mathiasiae (W.R. Anderson) W.R.

Anderson \& C. Davis

Bunchosia argentea (Jacq.) DC.

Bunchosia armeniaca (Cav.) DC.

Bunchosia hookeriana A. Juss.

Callaeum antifebrile (Griseb.) D.M. Johnson

Dicella julianii (J.F. Macbr.) W.R. Anderson

Diplopterys nutans (Poepp. ex Nied.) W.R. Anderson \& C.

Davis

Heteropterys brachiata (L.) DC.

Heteropterys tomentosa A. Juss.

Hiraea crassipes A. Juss.

Lophopterys inpana W.R. Anderson

Mascagnia schunkei W.R. Anderson

Stigmaphyllon argenteum C.E. Anderson

Stigmaphyllon bogotense Triana \& Planch.

Stigmaphyllon cardiophyllum A. Juss.

Stigmaphyllon florosum C.E. Anderson

Stigmaphyllon maynense Huber

Stigmaphyllon sinuatum (DC.) A. Juss.

Stigmaphyllon strigosum Poepp. ex A. Juss.

\section{Malvaceae}

Anoda cristata (L.) Schltdl.

Apeiba membranacea Spruce ex Benth.

Ayenia jussieui Cristóbal

Ayenia pusilla L.

Bastardia bivalvis (Cav.) Kunth ex Griseb.

Byttneria aculeata (Jacq.) Jacq.

Byttneria benensis Britton

Byttneria catalpifolia Jacq.

Byttneria hirsuta Ruiz \& Pav.

Callianthe geminiflora (Kunth) Donnell

Cavanillesia hylogeiton Ulbr.

Cavanillesia umbellata Ruiz \& Pav.

Ceiba insignis (Kunth) P.E. Gibbs \& Semir

Corchorus hirtus L.

Fuertesimalva limensis (L.) Fryxell

Gaya calyptrata (Cav.) Kunth ex K. Schum. 
Mendoncia lindavii Rusby

Mendoncia robusta Rusby

Mendoncia smithii Leonard

Pachystachys fosteri Wassh.

Pachystachys lutea Nees

Pachystachys spicata (Ruiz \& Pav.) Wassh.

Pseuderanthemum cordatum (Nees) Radlk.

Pseuderanthemum hookerianum (Nees) V.M. Baum

Pseuderanthemum lanceolatum (Ruiz \& Pav.) Wassh.

Pseuderanthemum weberbaueri Mildbr.

Pulchranthus adenostachyus (Lindau) V.M. Baum, Reveal

\& Nowicke

Ruellia brevifolia (Pohl) C. Ezcurra

Ruellia glischrocalyx Lindau

Ruellia haenkeana (Nees) Wassh.

Ruellia neoneesiana Wassh.

Ruellia pedunculosa (Nees) B.D. Jacks. \& Hook. f.

Ruellia proxima Lindau

Ruellia ruiziana (Nees) Lindau

Ruellia tarapotana Lindau

Ruellia yurimaguensis Lindau

Sanchezia aurea Leonard \& L.B. Sm.

Sanchezia coccinea Leonard \& L.B. Sm.

Sanchezia dasia Leonard \& L.B. Sm.

Sanchezia decora Leonard \& L.B. Sm.

Sanchezia ferreyrae Leonard \& L.B. Sm.

Sanchezia filamentosa Lindau

Sanchezia flava Leonard

Sanchezia lasia Leonard \& L.B. Sm.

Sanchezia longiflora (Hook.) Hook. f. ex Planch.

Sanchezia oblonga Ruiz \& Pav.

Sanchezia ovata Ruiz \& Pav.

Sanchezia pulchra Leonard

Sanchezia punicea Leonard \& L.B. Sm.

Sanchezia rubriflora Leonard

Sanchezia scandens (Lindau) Leonard \& L.B. Sm.

Sanchezia stenantha Leonard

Sanchezia stenomacra Leonard \& L.B. Sm.

Sanchezia woytkowskii Leonard \& L.B. Sm.

Sanchezia xantha Leonard \& L.B. Sm.

Stenostephanus longistaminus (Ruiz \& Pav.) V.M.

Baum

Stenostephanus macrolobus (Lindau) J.R.I. Wood

Stenostephanus sprucei (Lindau) Wassh. \& J.R.I.

Wood

Streblacanthus amoenus (Bremek.) T.F. Daniel

Streblacanthus dubiosus (Lindau) V.M. Baum

Teliostachya lanceolata Nees

Thunbergia alata Bojer ex Sims

\section{Achariaceae}

Carpotroche longifolia (Poepp.) Benth.
Gaya nutans (L'HÃ@r.) Sweet

Gaya weberbaueri Ulbr.

Gossypium barbadense L.

Guazuma crinita Mart.

Guazuma ulmifolia Lam.

Heliocarpus americanus L.

Herissantia crispa (L.) Brizicky

Hibiscus rosa-sinensis $\mathrm{L}$.

Luehea grandiflora Mart.

Luehea paniculata Mart.

Luehea speciosa Willd.

Malachra alceifolia Jacq.

Malachra ruderalis Gürke

Malva verticillata $\mathrm{L}$.

Malvastrum coromandelianum (L.) Garcke

Malvastrum scoparioides Ulbr.

Malvastrum tomentosum (L.) S.R. Hill

Malvaviscus penduliflorus DC.

Matisia bicolor Ducke

Matisia cordata Bonpl.

Matisia lasiocalyx K. Schum.

Melochia lupulina Sw.

Melochia mollis (K. Schum.) Hutch. \& Dalziel

Melochia pilosa (Mill.) Fawc. \& Rendle

Melochia thymifolia (C. Presl) Goldberg

Nototriche antoniana M. Chanco

Nototriche aretioides A.W. Hill

Nototriche gracilens Killip \& J.F. Macbr.

Nototriche longirostris (Wedd.) A.W. Hill

Nototriche pinnata (Cav.) A.W. Hill

Ochroma pyramidale (Cav. ex Lam.) Urb.

Pachira paraensis (Ducke) W.S. Alverson

Pachira punga-schunkei Fern. Alonso

Patinoa sphaerocarpa Cuatrec.

Pavonia communis A. St.-Hil.

Pavonia fruticosa (Mill.) Fawc. \& Rendle

Pavonia leucantha Poepp. ex Garcke

Pavonia oxyphyllaria Donn. Sm.

Pavonia paniculata Cav.

Pavonia peruviana Gürke

Pavonia sepium A. St.-Hil.

Pavonia spinifex (L.) Cav.

Pseudobombax septenatum (Jacq.) Dugand

Pterygota amazonica L.O. Williams ex Dorr

Quararibea wittii K. Schum. \& Ulbr.

Sida acuta Burm. f.

Sida rhombifolia L.

Sida setosa Mart. ex Colla 
Lindackeria paludosa (Benth.) Gilg

Mayna odorata Aubl.

\section{Actinidiaceae}

Saurauia biserrata (Ruiz \& Pav.) Spreng.

Saurauia bullosa Wawra

Saurauia formosa Sleumer

Saurauia glabra (Ruiz \& Pav.) Soejarto

Saurauia peruviana Buscal.

\section{Adoxaceae}

Sambucus canadensis L.

Sambucus peruviana Kunth

Viburnum hallii (Oerst.) Killip \& A.C. Sm.

Viburnum mathewsii (Oerst.) Killip \& A.C. Sm.

Viburnum reticulatum (Ruiz \& Pav. ex Oerst.) Killip

Viburnum seemenii Graebn.

Viburnum triphyllum Benth.

\section{Aizoaceae}

Aptenia cordifolia (L. f.) Schwantes

\section{Alismataceae}

Echinodorus horizontalis Rataj

\section{Alstroemeriaceae}

Alstroemeria pelegrina L.

Bomarea angustissima Killip

Bomarea aurantiaca Herb.

Bomarea brevis (Herb.) Baker

Bomarea campylophylla Killip

Bomarea coccinea (Ruiz \& Pav.) Baker

Bomarea cordifolia Herb.

Bomarea cornuta Herb.

Bomarea crocea (Ruiz \& Pav.) Herb.

Bomarea densiflora Herb.

Bomarea denticulata (Ruiz \& Pav.) Herb.

Bomarea dispar Herb.

Bomarea distichifolia (Ruiz \& Pav.) Baker

Bomarea dolichocarpa Killip

Bomarea dulcis (Hook.) Beauverd

Bomarea edulis (Tussac) Herb.

Bomarea endotrachys Kraenzl.

Bomarea ferreyrae Vargas

Bomarea formosissima (Ruiz \& Pav.) Herb.

Bomarea nematocaulon Killip

Bomarea ovata (Cav.) Mirb.

Bomarea pardina Herb.

Bomarea rosea (Ruiz \& Pav.) Herb.

Bomarea secundifolia (Ruiz \& Pav.) Baker

Bomarea setacea (Ruiz \& Pav.) Herb.
Sida spinosa L.

Sida urens $\mathrm{L}$.

Spirotheca rosea (Seem.) P.E. Gibbs \& W.S. Alverson

Sterculia apetala (Jacq.) H. Karst.

Sterculia frondosa Rich.

Sterculia stipulifera Ducke

Tarasa cerratei Krapov.

Theobroma cacao L.

Theobroma obovatum Klotzsch ex Bernoulli

Theobroma speciosum Willd. ex Spreng.

Theobroma subincanum Mart.

Triumfetta calycina Turcz.

Triumfetta grandiflora Vahl

Triumfetta lappula L.

Triumfetta semitriloba Jacq.

Urena lobata L.

Waltheria ovata Cav.

Wissadula contracta (Link) R.E. Fr.

Wissadula excelsior (Cav.) C. Presl

Wissadula microcarpa R.E. Fr.

Wissadula stellata (Cav.) K. Schum.

\section{Marantaceae}

Calathea altissima (Poepp. \& Endl.) Körn.

Calathea inocephala (Kuntze) H. Kenn. \& Nicolson

Calathea lateralis (Ruiz \& Pav.) Lindl.

Calathea lutea (Aubl.) Schult.

Goeppertia chrysoleuca (Poepp. \& Endl.) Borchs. \& S.

Suárez

Goeppertia dicephala (Poepp. \& Endl.) Borchs. \& S. Suárez

Goeppertia loeseneri (J.F. Macbr.) Borchs. \& S. Suárez

Goeppertia microcephala (Poepp. \& Endl.) Borchs. \& S

Suárez

Goeppertia pachystachya (Poepp. \& Endl.) Borchs. \& S.

Suárez

Goeppertia pavonii (Körn.) Borchs. \& S. Suárez Goeppertia peruviana (Körn.) Borchs. \& S. Suárez Goeppertia roseopicta (Linden) Borchs. \& S. Suárez Goeppertia sophiae (Huber) Borchs. \& S. Suárez Goeppertia standleyi (J.F. Macbr.) Borchs. \& S.

Suárez

Goeppertia undulata (Linden \& André) Borchs. \& S.

Suárez

Hylaeanthe unilateralis (Poepp. \& Endl.) A.M.E.

Jonker \& Jonker

Ischnosiphon arouma (Aubl.) Körn.

Ischnosiphon cerotus Loes.

Ischnosiphon gracilis (Rudge) Körn.

Ischnosiphon lasiocoleus Schum. ex Loes.

Ischnosiphon leucophaeus (Poepp. \& Endl.) Körn.

Ischnosiphon longiflorus K. Schum.

Ischnosiphon puberulus Loes.

Maranta arundinacea L. 
Bomarea speciosa Killip

Bomarea tarmensis Kraenzl.

\section{Alzateaceae}

Alzatea verticillata Ruiz \& Pav.

\section{Amaranthaceae}

Alternanthera bettzickiana (Regel) G. Nicholson

Alternanthera brasiliana (L.) Kuntze

Alternanthera elongata (Willd. ex Roem. \& Schult.)

Schinz

Alternanthera flavescens Kunth

Alternanthera halimifolia (Lam.) Standl. ex Pittier

Alternanthera lupulina Kunth

Alternanthera macbridei Standl.

Alternanthera philoxeroides (Mart.) Griseb.

Alternanthera porrigens (Jacq.) Kuntze

Alternanthera pungens Kunth

Alternanthera villosa Kunth

Amaranthus caudatus $\mathrm{L}$.

Amaranthus hybridus L.

Amaranthus spinosus $\mathrm{L}$.

Amaranthus viridis $\mathrm{L}$.

Celosia argentea $\mathrm{L}$.

Celosia grandifolia Moq.

Chamissoa acuminata Mart.

Chamissoa altissima (Jacq.) Kunth

Chenopodium petiolare Kunth

Cyathula achyranthoides (Kunth) Moq.

Dysphania ambrosioides (L.) Mosyakin \& Clemants

Gomphrena elegans Mart.

Gomphrena globosa L.

Gomphrena vaga Mart.

Guilleminea densa (Humb. \& Bonpl. ex Schult.) Moq.

Hebanthe erianthos (Poir.) Pedersen

Iresine diffusa Humb. \& Bonpl. ex Willd.

Iresine mexicana (Moq.) Suess.

Iresine weberbaueri Suess.

Pedersenia hassleriana (Chodat) Pedersen

\section{Amaryllidaceae}

Eucharis amazonica Linden ex Planch.

Eucharis oxyandra (Ravenna) Ravenna

Eustephia coccinea Cav.

Eustephia longibracteata Vargas

Hippeastrum puniceum (Lam.) Voss

Stenomesson coccineum Herb.

Stenomesson pearcei Baker

Urceolina microcrater Kraenzl.

\section{Anacardiaceae}

Anacardium occidentale L.

Astronium lecointei Ducke

Mauria ferruginea Tul.
Maranta ruiziana Körn.

Monotagma angustissimum Loes.

Monotagma juruanum Loes.

Monotagma laxum (Poepp. \& Endl.) Schum.

Monotagma plurispicatum (Körn.) K. Schum.

\section{Marattiaceae}

Danaea elliptica Sm.

Danaea moritziana C. Presl

Danaea nodosa (L.) Sm.

\section{Marcgraviaceae}

Marcgravia crenata Poepp. ex Wittm.

Marcgraviastrum mixtum (Triana \& Planch.) Bedell

Norantea guianensis Aubl.

Norantea obovata Ruiz \& Pav. ex G. Don

Ruyschia pavonii G. Don

Schwartzia magnifica (Gilg) Bedell

Souroubea didyma (Poepp. ex Wittm.) Gilg

Souroubea peruviana Gilg

\section{Melastomataceae}

Aciotis purpurascens (Aubl.) Triana

Aciotis rubricaulis (Mart. ex DC.) Triana

Adelobotrys acreana Wurdack

Adelobotrys adscendens (Sw.) Triana

Adelobotrys boissierianus Cogn.

Adelobotrys tessmannii Markgr.

Alloneuron majus (Markgr.) Markgr. ex J.F. Macbr.

Arthrostemma ciliatum Pav. ex D. Don

Axinaea glandulosa Ruiz \& Pav. ex D. Don

Axinaea lanceolata Ruiz \& Pav.

Axinaea nitida Cogn.

Axinaea weberbaueri Cogn.

Bellucia aequiloba Pilg.

Bellucia pentamera Naudin

Bellucia spruceana (Benth. ex Triana) J.F. Macbr.

Bellucia subandina (Wurdack) Penneys, Michelang.,

Judd \& Almeda

Bellucia umbellata Gleason

Blakea latifolia (Ruiz \& Pav.) D. Don

Blakea mexiae Gleason

Blakea ovalis (Ruiz \& Pav.) D. Don

Blakea repens (Ruiz \& Pav.) D. Don

Blakea rosea (Ruiz \& Pav.) D. Don

Blakea sawadae J.F. Macbr.

Blakea spruceana Cogn.

Blakea standleyana J.F. Macbr.

Brachyotum campanulare (Bonpl.) Triana

Brachyotum huancavelicae Wurdack

Brachyotum lutescens (Ruiz \& Pav.) Triana

Brachyotum lycopodioides Triana

Brachyotum markgrafii Wurdack 
Mauria heterophylla Kunth

Mauria peruviana Cuatrec.

Mauria sericea Loes.

Mauria simplicifolia Kunth

Schinus molle L.

Spondias mombin L.

Spondias purpurea $\mathrm{L}$.

Tapirira guianensis Aubl.

Toxicodendron striatum (Ruiz \& Pav.) Kuntze

Anemiaceae

Anemia flexuosa (Savigny) Sw.

Anemia hirsuta (L.) Sw.

Anemia pastinacaria Moritz ex Prantl

Annonaceae

Anaxagorea dolichocarpa Sprague \& Sandwith

Annona ambotay Aubl.

Annona cherimola Mill.

Annona edulis (Triana \& Planch.) H. Rainer

Annona hispida (Maas \& Westra) H. Rainer

Annona hypoglauca Mart.

Annona jucunda (Diels) H. Rainer

Annona neoulei $\mathrm{H}$. Rainer

Annona scandens Diels

Annona schunkei (Maas \& Westra) H. Rainer

Cremastosperma cauliflorum R.E. Fr.

Cremastosperma pedunculatum (Diels) R.E. Fr.

Cymbopetalum longipes Benth. ex Diels

Cymbopetalum schunkei N.A. Murray

Duguetia macrophylla R.E. Fr.

Duguetia quitarensis Benth.

Fusaea longifolia (Aubl.) Saff.

Guatteria blepharophylla Mart.

Guatteria eriopoda DC.

Guatteria excelsa Poepp. ex Mart.

Guatteria glauca Ruiz \& Pav.

Guatteria hirsuta Ruiz \& Pav.

Guatteria megalophylla Diels

Guatteria modesta Diels

Guatteria olivacea R.E. Fr.

Guatteria pteropus Benth.

Guatteria ucayalina Huber

Klarobelia pumila Chatrou

Malmea pachiteae D.R. Simpson

Malmea peruviana R.E. Fr.

Malmea raimondii R.E. Fr.

Mosannona pachiteae (D.R. Simpson) Chatrou

Oxandra mediocris Diels

Oxandra xylopioides Diels

Porcelia nitidifolia Ruiz \& Pav.

Porcelia ponderosa (Rusby) Rusby
Brachyotum naudinii Triana

Brachyotum quinquenerve (Ruiz \& Pav.) Triana

Brachyotum rosmarinifolium (Ruiz \& Pav.) Triana

Brachyotum sanguinolentum (Naudin) Triana

Brachyotum tyrianthinum J.F. Macbr.

Centronia laurifolia D. Don

Centronia peruviana J.F. Macbr.

Centronia reticulata Triana

Clidemia allardii Wurdack

Clidemia capitellata (Bonpl.) D. Don

Clidemia cordata Cogn. ex Britton

Clidemia dentata Pav. ex D. Don

Clidemia dimorphica J.F. Macbr.

Clidemia epiphytica (Triana) Cogn.

Clidemia graciliflora Huber

Clidemia heterophylla (Desr.) Gleason

Clidemia hirta (L.) D. Don

Clidemia longifolia Gleason

Clidemia obliqua Cogn.

Clidemia octona (Bonpl.) L.O. Williams

Clidemia piperifolia Gleason

Clidemia rubra (Aubl.) Mart.

Clidemia septuplinervia Cogn.

Clidemia serpens (Triana) Cogn.

Clidemia sessiliflora (Naudin) Cogn.

Clidemia sprucei Gleason

Clidemia strigillosa (Sw.) DC.

Conostegia inusitata Wurdack

Conostegia superba D. Don ex Naudin

Graffenrieda cucullata (Triana) L.O. Williams

Graffenrieda emarginata (Ruiz \& Pav.) Triana

Graffenrieda foliosa Cogn.

Graffenrieda intermedia Triana

Graffenrieda limbata Triana

Graffenrieda miconioides Naudin

Graffenrieda robusta (Cogn.) L.O. Williams

Henriettea aggregata (D. Don) J.F. Macbr.

Leandra candelabrum (J.F. Macbr.) Wurdack

Leandra chaetodon (DC.) Cogn.

Leandra clidemioides (Naudin) Wurdack

Leandra crenata (D. Don) Cogn.

Leandra dichotoma (Pav. ex D. Don) Cogn.

Leandra francavillana Cogn.

Leandra longicoma Cogn.

Leandra nervosa (Naudin) Cogn.

Leandra retropila Cogn.

Leandra secunda (D. Don) Cogn.

Loreya arborescens (Aubl.) DC.

Maieta guianensis Aubl.

Maieta poeppigii Mart. ex Cogn. 
Rollinia mucosa (Jacq.) Baill.

Ruizodendron ovale (Ruiz \& Pav.) R.E. Fr.

Tetrameranthus umbellatus Westra

Unonopsis guatterioides (A. DC.) R.E. Fr.

Xylopia conjungens R.E. Fr.

Xylopia cuspidata Diels

Xylopia densiflora R.E. Fr.

Xylopia sericea A. St.-Hil.

\section{Apiaceae}

Arracacia elata H. Wolff

Arracacia incisa $\mathrm{H}$. Wolff

Arracacia xanthorrhiza Bancr.

Azorella compacta Phil.

Azorella corymbosa (Ruiz \& Pav.) Pers.

Azorella crenata (Ruiz \& Pav.) Pers.

Azorella diapensioides A. Gray

Azorella multifida (Ruiz \& Pav.) Pers.

Azorella pulvinata Wedd.

Bowlesia flabilis J.F. Macbr.

Conium maculatum L.

Cryptotaenia canadensis (L.) DC.

Cyclospermum leptophyllum (Pers.) Sprague ex

Britton \& P. Wilson

Daucus montanus Humb. \& Bonpl. ex Spreng.

Eryngium foetidum L.

Eryngium humile Cav.

Eryngium nudicaule Lam.

Lilaeopsis macloviana (Gand.) A.W. Hill

Niphogeton scabra (H. Wolff) J.F. Macbr.

Oreomyrrhis andicola (Kunth) Endl. ex Hook. f.

Ridolfia segetum (L.) Moris

Sanicula liberta Cham. \& Schltdl.

Spananthe paniculata Jacq.

\section{Apocynaceae}

Allamanda cathartica $\mathrm{L}$.

Allomarkgrafia ovalis (Ruiz \& Pav. ex Markgr.)

Woodson

Allomarkgrafia tubiflora Woodson ex Dwyer

Asclepias curassavica L.

Aspidosperma capitatum L.O. Williams

Aspidosperma cylindrocarpon Müll. Arg.

Aspidosperma macrocarpon Mart.

Aspidosperma parvifolium A. DC.

Aspidosperma rigidum Rusby

Blepharodon salicinum Decne.

Cascabela thevetia (L.) Lippold

Couma macrocarpa Barb. Rodr.

Cynanchum formosum N.E. Br.

Cynanchum longirostrum (K. Schum.) W.D. Stevens

Cynanchum montevidense Spreng.

Cynanchum tarmense Schltr.
Meriania amischophylla Wurdack

Meriania radula (Benth.) Triana

Meriania weberbaueri J.F. Macbr.

Miconia adrieni J.F. Macbr.

Miconia affinis DC.

Miconia albicans (Sw.) Steud.

Miconia alpina Cogn.

Miconia amazonica Triana

Miconia amplexicaulis Naudin

Miconia atrofusca Cogn.

Miconia aulocalyx Mart. ex Triana

Miconia aurea (D. Don) Naudin

Miconia aureoides Cogn.

Miconia bailloniana J.F. Macbr.

Miconia barbeyana Cogn.

Miconia barbinervis (Benth.) Triana

Miconia biglandulosa Gleason

Miconia brachyanthera Triana

Miconia brevistylis Cogn.

Miconia bubalina (D. Don) Naudin

Miconia caerulea (Pav. ex D. Don) Naudin

Miconia calvescens DC.

Miconia carpishana Wurdack

Miconia centrodesma Naudin

Miconia centrodesmoides Wurdack

Miconia choriophylla Wurdack

Miconia ciliata (Rich.) DC.

Miconia clathrantha Triana ex Cogn.

Miconia coelestis (Pav. ex D. Don) Naudin

Miconia crassistigma Cogn.

Miconia cyanocarpa Naudin

Miconia decurrens Cogn.

Miconia densifolia Cogn.

Miconia dipsacea Naudin

Miconia dispar Benth.

Miconia dodecandra Cogn.

Miconia dolichorrhyncha Naudin

Miconia doniana Naudin

Miconia dumetosa Cogn.

Miconia elaeagnoides (Sw.) Griseb.

Miconia erioclada Triana

Miconia fictilis (J.F. Macbr.) Michelang. \& R. Goldenb.

Miconia flaccida Gleason

Miconia fruticulosa Cogn.

Miconia galactantha Naudin

Miconia glandulifera Cogn.

Miconia glomerata Triana

Miconia griffisii J.F. Macbr.

Miconia grisea Cogn. 
Ditassa crassa Schltr.

Fischeria stellata (Vell.) E. Fourn.

Forsteronia graciloides Woodson

Geissospermum reticulatum A.H. Gentry

Himatanthus articulatus (Vahl) Woodson

Laubertia boissieri A. DC.

Macropharynx spectabilis (Stadelm.) Woodson

Mandevilla cercophylla Woodson

Mandevilla glandulosa (Ruiz \& Pav.) Woodson

Mandevilla laxa (Ruiz \& Pav.) Woodson

Mandevilla pavonii (A. DC.) Woodson

Mandevilla polyantha K. Schum. ex Woodson

Mandevilla sandemanii Woodson

Mandevilla subsagittata (Ruiz \& Pav.) Woodson

Mandevilla trianae Woodson

Matelea glandulosa (Poepp. ex Decne.) Morillo

Matelea longifolia (Markgr.) Morillo

Matelea peruviana Morillo

Matelea rivularis Woodson

Mesechites acuminatus (Ruiz \& Pav.) Müll. Arg.

Mesechites trifidus (Jacq.) Müll. Arg.

Metalepis albiflora Urb.

Nerium oleander L.

Odontadenia verrucosa (Willd. ex Roem. \& Schult.)

K. Schum. ex Markgr.

Oxypetalum erianthum Decne.

Oxypetalum retusum (Markgr.) Goyder

Peltastes peruvianus Woodson

Philibertia campanulata (Lindl.) G. Nicholson

Plumeria rubra L.

Prestonia denticulata (Vell.) Woodson

Prestonia quinquangularis (Jacq.) Spreng.

Rauvolfia sprucei Müll. Arg.

Secondatia densiflora A. DC.

Stenomeria decalepis Turcz.

Tabernaemontana columbiensis (L. Allorge)

Leeuwenb.

Tabernaemontana coriacea Link ex Roem. \& Schult.

Tabernaemontana flavicans Willd. ex Roem. \&

Schult.

Tabernaemontana sananho Ruiz \& Pav.

Tabernaemontana siphilitica (L. f.) Leeuwenb.

Tabernaemontana undulata Vahl

Tabernaemontana vanheurckii Müll. Arg.

Tassadia berteroana (Spreng.) W.D. Stevens

\section{Aquifoliaceae}

llex aggregata (Ruiz \& Pav.) Loes.

Ilex hippocrateoides Kunth

Ilex microsticta Loes.

Ilex ovalis (Ruiz \& Pav.) Loes.

Ilex tarapotina Loes.
Miconia heteromera Naudin

Miconia hexapetala Wurdack

Miconia holosericea (L.) DC.

Miconia hookeriana Triana

Miconia hospitalis Wurdack

Miconia huanucensis Wurdack

Miconia ibaguensis (Bonpl.) Triana

Miconia icosandra Gleason

Miconia koepckeana Wurdack

Miconia lamprophylla Triana

Miconia lasiocalyx Cogn.

Miconia lasiostyla Gleason

Miconia latifolia (D. Don) Naudin

Miconia laurina (D. Don) Naudin

Miconia laxivenula (Wurdack) Gamba \& Almeda

Miconia livida Triana

Miconia longifolia (Aubl.) DC.

Miconia lugubris Cogn.

Miconia malatestae J.F. Macbr.

Miconia martiniana Gleason

Miconia matthaei Naudin

Miconia media (D. Don) Naudin

Miconia membranacea Triana

Miconia miles-morganii J.F. Macbr.

Miconia minutiflora (Bonpl.) DC.

Miconia monzoniensis Cogn.

Miconia muricata (D. Don) Triana

Miconia myriantha Benth.

Miconia neomicrantha Judd \& Skean

Miconia neriifolia Triana

Miconia nervosa (Sm.) Triana

Miconia nigricans Cogn.

Miconia nutans Donn. Sm.

Miconia obscura (Bonpl.) Naudin

Miconia opacifolia J.F. Macbr.

Miconia ottikeri J.F. Macbr.

Miconia paeminosa Wurdack

Miconia paleacea Cogn.

Miconia pavoniana Naudin

Miconia phaeophylla Triana

Miconia poeppigii Triana

Miconia polytopica Wurdack

Miconia prancei Wurdack

Miconia procumbens (Gleason) Wurdack

Miconia pterocaulon Triana

Miconia pulgari J.F. Macbr.

Miconia pulverulenta Ruiz \& Pav.

Miconia punctata (Desr.) D. Don ex DC. 
Ilex teratopis Loes.

Ilex uniflora Benth.

Ilex villosula Loes.

\section{Araceae}

Anthurium amoenum Kunth \& Bouché

Anthurium apaporanum R.E. Schultes

Anthurium atropurpureum R.E. Schult. \& Maguire

Anthurium brevipedunculatum Madison

Anthurium breviscapum Kunth

Anthurium clavigerum Poepp.

Anthurium consimile Schott

Anthurium corallinum Poepp.

Anthurium croatii Madison

Anthurium dombeyanum Brongn. ex Schott

Anthurium eminens Schott

Anthurium ernestii Engl.

Anthurium flavescens Poepp.

Anthurium formosum Schott

Anthurium gracile (Rudge) Schott

Anthurium gracilipedunculatum K. Krause

Anthurium huallagense Engl.

Anthurium huanucense Engl.

Anthurium kunthii Poepp.

Anthurium latissimum Engl.

Anthurium lechlerianum Schott

Anthurium Ilewelynii Croat

Anthurium lutescens Engl.

Anthurium macbridei K. Krause

Anthurium macleanii Schott

Anthurium monzonense Engl.

Anthurium obtusum (Engl.) Grayum

Anthurium oxycarpum Poepp.

Anthurium pedatoradiatum Schott

Anthurium peltatum Poepp.

Anthurium polydactylum Madison

Anthurium reflexinervium Croat

Anthurium rubrinervium (Link) G. Don

Anthurium scandens (Aubl.) Engl.

Anthurium siccisilvarum K. Krause

Anthurium soukupii Croat

Anthurium truncicola Engl.

Anthurium uleanum Engl.

Anthurium weberbaueri Engl.

Caladium bicolor (Aiton) Vent.

Dieffenbachia costata H. Karst. ex Schott

Dieffenbachia macrophylla Poepp.

Dieffenbachia weberbaueri Engl.

Dracontium spruceanum (Schott) G.H. Zhu

Epipremnum pinnatum (L.) Engl.

Gorgonidium vargasii Bogner \& Nicolson
Miconia quadrifolia Naudin

Miconia radula Cogn.

Miconia reflexipila Wurdack

Miconia rotundifolia (D. Don) Naudin

Miconia rugifolia Triana

Miconia saltuensis Wurdack

Miconia sanguinea (D. Don) Triana

Miconia saxatilis J.F. Macbr.

Miconia serrulata (DC.) Naudin

Miconia spennerostachya Naudin

Miconia splendens (Sw.) Griseb.

Miconia stelligera Cogn.

Miconia stenostachya DC.

Miconia terera Naudin

Miconia ternatifolia Triana

Miconia tetragona Cogn.

Miconia tetrasperma Gleason

Miconia thaminantha Wurdack

Miconia tiri Triana

Miconia tomentosa (Rich.) D. Don ex DC.

Miconia trichogona J.F. Macbr.

Miconia triplinervis Ruiz \& Pav.

Miconia variabilis Gamba \& Almeda

Miconia venulosa Wurdack

Miconia vitiflora J.F. Macbr.

Miconia wagneri J.F. Macbr.

Miconia weberbaueri Cogn.

Miconia wolfei Wurdack

Miconia woytkowskii Wurdack

Monochaetum dicranantherum Naudin

Monochaetum lineatum (D. Don) Naudin

Monochaetum subditivum J.F. Macbr.

Monolena primuliflora Hook. f.

Mouriri myrtilloides (Sw.) Poir.

Ossaea boliviensis (Cogn.) Gleason

Ossaea bullifera (Pilg.) Gleason

Ossaea capillaris (D. Don) Cogn.

Ossaea cucullata Gleason

Ossaea quadrisulca (Naudin) Wurdack

Salpinga peruviana (Cogn.) Wurdack

Tibouchina asperifolia Cogn.

Tibouchina echinata (Ruiz \& Pav.) Cogn.

Tibouchina lepidota (Bonpl.) Baill.

Tibouchina longifolia (Vahl) Baill.

Tibouchina ochypetala (Ruiz \& Pav.) Baill.

Tibouchina pleromoides J.F. Macbr.

Tibouchina repens Wurdack

Tibouchina saxosa Gleason

Tibouchina solmsii Cogn.

Tococa caquetana Sprague 
Homalomena peltata Mast.

Incarum pavonii (Schott) E.G. Gonç.

Landoltia punctata (G. Mey.) Les \& D.J. Crawford

Lemna gibba L.

Lemna minuta Kunth

Monstera adansonii Schott

Monstera dubia (Kunth) Engl. \& K. Krause

Monstera lechleriana Schott

Monstera obliqua Miq.

Monstera subpinnata (Schott) Engl.

Philodendron acreanum K. Krause

Philodendron alatum Poepp.

Philodendron angustialatum Engl.

Philodendron barrosoanum G.S. Bunting

Philodendron chinchamayense Engl.

Philodendron cruentum Poepp.

Philodendron deltoideum Poepp.

Philodendron densivenium Engl.

Philodendron divaricatum K. Krause

Philodendron ernestii Engl.

Philodendron exile G.S. Bunting

Philodendron fibrillosum Poepp.

Philodendron guttiferum Kunth

Philodendron hederaceum (Jacq.) Schott

Philodendron heterophyllum Poepp.

Philodendron huanucense Engl.

Philodendron hylaeae G.S. Bunting

Philodendron juninense Engl.

Philodendron lechlerianum Schott

Philodendron mathewsii Schott

Philodendron megalophyllum Schott

Philodendron membranaceum Poepp.

Philodendron micranthum Poepp. ex Schott

Philodendron ornatum Schott

Philodendron ruizii Schott

Philodendron stenophyllum K. Krause

Pistia stratiotes L.

Rhodospatha latifolia Poepp.

Rhodospatha oblongata Poepp.

Stenospermation amomifolium (Poepp.) Schott

Stenospermation crassifolium Engl.

Stenospermation flavescens Engl.

Stenospermation multiovulatum (Engl.) N.E. Br.

Stenospermation spruceanum Schott

Stenospermation wallisii Mast.

Stenospermation zeacarpium Madison

Syngonium gentryanum Croat

Xanthosoma fractum Madison

Xanthosoma pubescens Poepp.

Xanthosoma stenospathum Madison
Tococa carolensis Gleason

Tococa guianensis Aubl.

Tococa parviflora Spruce ex Triana

Tococa stenoptera Gleason

Tococa stephanotricha Naudin

Triolena allardii (Wurdack) Wurdack

Triolena amazonica (Pilg.) Wurdack

\section{Meliaceae}

Cabralea canjerana (Vell.) Mart.

Cedrela fissilis Vell.

Cedrela odorata L.

Cedrela weberbaueri Harms

Guarea gomma Pulle

Guarea guidonia (L.) Sleumer

Guarea kunthiana A. Juss.

Guarea macrophylla Vahl

Guarea pterorhachis Harms

Guarea pubescens (Rich.) A. Juss.

Melia azedarach L.

Swietenia macrophylla King

Trichilia elegans A. Juss.

Trichilia inaequilatera T.D. Penn.

Trichilia maynasiana C. DC.

Trichilia micrantha Benth.

Trichilia pallida Sw.

Trichilia pleeana (A. Juss.) C. DC.

Trichilia poeppigii C. DC.

Trichilia quadrijuga Kunth

Trichilia rubra C. DC.

Trichilia septentrionalis C. DC.

Trichilia tuberculata (Triana \& Planch.) C. DC.

\section{Menispermaceae}

Abuta grandifolia (Mart.) Sandwith

Abuta pahnii (Mart.) Krukoff \& Barneby

Abuta rufescens Aubl.

Abuta solimoesensis Krukoff \& Barneby

Abuta velutina Gleason

Anomospermum bolivianum Krukoff \& Moldenke

Anomospermum chloranthum Diels

Anomospermum grandifolium Eichler

Anomospermum reticulatum (Mart.) Eichler

Borismene japurensis (Mart.) Barneby

Chondrodendron tomentosum Ruiz \& Pav.

Cissampelos andromorpha DC.

Cissampelos fasciculata Benth.

Cissampelos grandifolia Triana \& Planch.

Cissampelos laxiflora Moldenke

Cissampelos pareira L.

Cissampelos tropaeolifolia DC.

Curarea tecunarum Barneby \& Krukoff 
Araliaceae

Dendropanax tessmannii (Harms) Harms

Dendropanax umbellatus (Ruiz \& Pav.) Decne. \&

Planch.

Dendropanax weberbaueri (Harms) Harms

Hydrocotyle acutifolia Ruiz \& Pav.

Hydrocotyle alchemilloides A. Rich.

Hydrocotyle bonariensis Lam.

Hydrocotyle bonplandii A. Rich.

Hydrocotyle dahlgrenii Rose \& J.F. Macbr.

Hydrocotyle globiflora Ruiz \& Pav.

Hydrocotyle gracilis Ruiz \& Pav.

Hydrocotyle pusilla A. Rich.

Hydrocotyle quinqueloba Ruiz \& Pav.

Hydrocotyle steyermarkii Mathias \& Constance

Oreopanax aquifolius Harms

Oreopanax argentatus (Kunth) Decne. \& Planch.

Oreopanax capitatus (Jacq.) Decne. \& Planch.

Oreopanax iodophyllus Harms

Oreopanax pavonii Seem.

Oreopanax polycephalus Harms

Oreopanax ruizii Decne. \& Planch. ex Harms

Schefflera acuminata (Ruiz \& Pav.) Harms

Schefflera angulata (Pav.) Harms

Schefflera japurensis (Mart. \& Zucc. ex Marchal) Harms

Schefflera minutiflora Harms

Schefflera monzonensis Harms

Schefflera morototoni (Aubl.) Maguire, Steyerm. \& Frodin

Schefflera pedicellata (Ruiz \& Pav.) Harms

Schefflera pentandra (Pav.) Harms

Schefflera violacea Cuatrec.

\section{Arecaceae}

Aiphanes deltoidea Burret

Aiphanes horrida (Jacq.) Burret

Aiphanes weberbaueri Burret

Attalea phalerata Mart. ex Spreng.

Bactris concinna Mart.

Bactris maraja Mart.

Ceroxylon vogelianum (Engel) $\mathrm{H}$. Wendl.

Chamaedorea angustisecta Burret

Chamaedorea fragrans (Ruiz \& Pav.) Mart.

Chamaedorea linearis (Ruiz \& Pav.) Mart.

Chamaedorea pauciflora Mart.

Chamaedorea pinnatifrons (Jacq.) Oerst.

Chelyocarpus ulei Dammer

Desmoncus orthacanthos Mart.

Euterpe precatoria Mart.

Geonoma brongniartii Mart.

Geonoma camana Trail

Geonoma interrupta (Ruiz \& Pav.) Mart.
Curarea toxicofera (Wedd.) Barneby \& Krukoff

Disciphania convolvulacea (Poepp.) Diels

Hyperbaena domingensis (DC.) Benth.

Odontocarya tripetala Diels

Orthomene schomburgkii (Miers) Barneby \& Krukoff

Sciadotenia eichleriana Moldenke

Telitoxicum krukovii Moldenke

\section{Metaxyaceae}

Metaxya rostrata (Humb. \& Bonpl. ex Willd.) C. Pres

\section{Monimiaceae}

Mollinedia killipii J.F. Macbr.

Mollinedia lanceolata Ruiz \& Pav.

Mollinedia ovata Ruiz \& Pav.

Mollinedia repanda Ruiz \& Pav.

\section{Montiaceae}

Calandrinia acaulis Kunth

Calandrinia ciliata (Ruiz \& Pav.) DC.

Montia fontana $\mathrm{L}$.

\section{Moraceae}

Batocarpus amazonicus (Ducke) Fosberg

Batocarpus orinocensis $\mathrm{H}$. Karst.

Brosimum acutifolium Huber

Brosimum alicastrum Sw.

Brosimum guianense (Aubl.) Huber

Brosimum lactescens (S. Moore) C.C. Berg

Brosimum multinervium C.C. Berg

Brosimum utile (Kunth) Oken

Clarisia biflora Ruiz \& Pav.

Clarisia racemosa Ruiz \& Pav.

Dorstenia brasiliensis Lam.

Dorstenia peruviana C.C. Berg

Ficus americana Aubl.

Ficus caballina Standl.

Ficus casapiensis (Miq.) Miq.

Ficus donnell-smithii Standl.

Ficus eximia Schott

Ficus insipida Willd.

Ficus macbridei Standl.

Ficus maxima Mill.

Ficus nymphaeifolia Mill.

Ficus paraensis (Miq.) Miq.

Ficus pertusa L. f.

Ficus schippii Standl.

Ficus sphenophylla Standl.

Ficus trigona L. f.

Ficus trigonata $\mathrm{L}$.

Helicostylis tomentosa (Poepp. \& Endl.) Rusby

Helicostylis tovarensis (Klotzsch \& H. Karst.) C.C.

Berg

Maclura tinctoria (L.) D. Don ex Steud. 
Geonoma leptospadix Trail Geonoma maxima (Poit.) Kunth

Geonoma orbignyana Mart.

Geonoma poeppigiana Mart.

Geonoma stricta (Poit.) Kunth

Geonoma trigona (Ruiz \& Pav.) A.H. Gentry

Geonoma undata Klotzsch

Hyospathe elegans Mart.

Hyospathe peruviana A.J. Hend.

Iriartea deltoidea Ruiz \& Pav.

Iriartella setigera (Mart.) $\mathrm{H}$. Wendl.

Mauritia flexuosa L. f.

Oenocarpus bataua Mart.

Phytelephas macrocarpa Ruiz \& Pav.

Prestoea ensiformis (Ruiz \& Pav.) H.E. Moore

Socratea exorrhiza (Mart.) H. Wendl.

Wendlandiella gracilis Dammer

Wettinia augusta Poepp. \& Endl.

Wettinia maynensis Spruce

\section{Aristolochiaceae}

Aristolochia dalyi F. González

Aristolochia fragrantissima Ruiz

Aristolochia ruiziana (Klotzsch) Duch.

Aristolochia schunkeana F. González

\section{Asparagaceae}

Agave americana $\mathrm{L}$.

Anthericum eccremorrhizum Ruiz \& Pav.

Furcraea andina Trel.

Furcraea occidentalis Trel.

\section{Aspleniaceae}

Asplenium abscissum Willd.

Asplenium angustum Sw.

Asplenium auritum Sw.

Asplenium cuspidatum Lam.

Asplenium dissectum Sw.

Asplenium divaricatum Kunze

Asplenium extensum Fée

Asplenium laetum Sw.

Asplenium nidus L.

Asplenium projectum Kunze

Asplenium pseudoangustum Stolze

Asplenium rutaceum (Willd.) Mett.

Asplenium serra Langsd. \& Fisch.

Asplenium serratum $\mathrm{L}$.

Asplenium sulcatum Lam.

\section{Asteraceae}

Achyrocline alata (Kunth) DC.

Achyrocline satureioides (Lam.) DC.

Adenostemma platyphyllum Cass.

Aequatorium carpishense (Cuatrec.) H. Rob. \&
Naucleopsis ulei (Warb.) Ducke

Perebea guianensis Aubl.

Perebea humilis C.C. Berg

Perebea rubra (Trécul) C.C. Berg

Poulsenia armata (Miq.) Standl.

Pseudolmedia laevigata Trécul

Pseudolmedia laevis (Ruiz \& Pav.) J.F. Macbr.

Pseudolmedia macrophylla Trécul

Sorocea briquetii J.F. Macbr.

Sorocea guilleminiana Gaudich.

Sorocea pubivena Hemsl.

Sorocea steinbachii C.C. Berg

Sorocea trophoides W.C. Burger

Trophis caucana (Pittier) C.C. Berg

Trophis racemosa (L.) Urb.

\section{Muntingiaceae}

Muntingia calabura L.

\section{Myricaceae}

Morella pubescens (Humb. \& Bonpl. ex Willd.) Wilbur

\section{Myristicaceae}

Compsoneura capitellata (A. DC.) Warb.

Compsoneura sprucei (A. DC.) Warb.

Iryanthera hostmannii (Benth.) Warb.

Iryanthera juruensis Warb.

Iryanthera paraensis Huber

Otoba parvifolia (Markgr.) A.H. Gentry

Virola decorticans Ducke

Virola pavonis (A. DC.) A.C. Sm.

Virola peruviana (A. DC.) Warb.

Virola sebifera Aubl.

Virola surinamensis (Rol. ex Rottb.) Warb.

\section{Myrtaceae}

Acca lanuginosa (Ruiz \& Pav. ex G. Don) McVaugh

Acca macrostema (Ruiz \& Pav. ex G. Don) McVaugh

Calyptranthes bipennis $\mathrm{O}$. Berg

Calyptranthes densiflora Poepp. ex O. Berg

Calyptranthes paniculata Grosourdy

Calyptranthes speciosa Sagot

Campomanesia speciosa (Diels) McVaugh

Eugenia biflora (L.) DC.

Eugenia egensis DC.

Eugenia ependytes McVaugh

Eugenia limbosa O. Berg

Eugenia patens Poir.

Eugenia racemiflora O. Berg

Eugenia subterminalis DC.

Myrcia atrorufa McVaugh

Myrcia bracteata (Rich.) DC.

Myrcia fallax (Rich.) DC.

Myrcia fascicularis O. Berg 
Cuatrec.

Aequatorium rimachianum (Cuatrec.) H. Rob. \&

Cuatrec.

Aequatorium stellatopilosum (Greenm. \& Cuatrec.)

C. Jeffrey

Aequatorium tuestae (Cuatrec.) H. Rob. \& Cuatrec. Aetheolaena campanulata (Sch. Bip. ex Klatt) B.

Nord.

Ageratina cutervensis (Hieron.) R.M. King \& H. Rob. Ageratina gilbertii (B.L. Rob.) R.M. King \& H. Rob. Ageratina glechonophylla (Less.) R.M. King \& H. Rob.

Ageratina gracilenta (B.L. Rob.) R.M. King \& H. Rob. Ageratina macbridei (B.L. Rob.) R.M. King \& H. Rob. Ageratina pichinchensis (Kunth) R.M. King \& H. Rob. Ageratina rhytidodes (B.L. Rob.) R.M. King \& H. Rob.

Ageratina scopulorum (Wedd.) R.M. King \& H. Rob. Ageratina sternbergiana (DC.) R.M. King \& H. Rob. Ageratina stictophylla (B.L. Rob.) R.M. King \& H.

Rob.

Ageratina tambillensis (Hieron.) R.M. King \& H. Rob.

Ageratina vallincola (DC.) R.M. King \& H. Rob.

Ageratum conyzoides $\mathrm{L}$.

Ambrosia arborescens Mill.

Ambrosia artemisiifolia L.

Antennaria linearifolia Wedd.

Aphanactis villosa S.F. Blake

Aristeguietia diplodictyon (B.L. Rob.) R.M. King \& H. Rob.

Aristeguietia discolor R.M. King \& H. Rob.

Aristeguietia gascae (B.L. Rob.) R.M. King \& H. Rob.

Aspilia foliacea (Spreng.) Baker

Asplundianthus scabrifolius (B.L. Rob.) R.M. King \&

H. Rob.

Austroeupatorium decemflorum (DC.) R.M. King \&

H. Rob.

Austroeupatorium inulaefolium (Kunth) R.M.King \&

H.Rob.

Ayapana amygdalina (Lam.) R.M. King \& H. Rob.

Ayapana elata (Steetz) R.M. King \& H. Rob.

Baccharis caespitosa (Ruiz \& Pav.) Pers.

Baccharis corymbosa (Ruiz \& Pav.) Pers.

Baccharis dependens Pers.

Baccharis emarginata (Ruiz \& Pav.) Pers.

Baccharis genistelloides (Lam.) Pers.

Baccharis latifolia (Ruiz \& Pav.) Pers.

Baccharis linearifolia (Lam.) Pers.

Baccharis nitida (Ruiz \& Pav.) Pers.

Baccharis oblongifolia (Ruiz \& Pav.) Pers.

Baccharis pedunculata (Mill.) Cabrera

Baccharis salicina Torr. \& A. Gray
Myrcia mollis (Kunth) DC.

Myrcia multiflora (Lam.) DC.

Myrcia platycaula Diels

Myrcia splendens (Sw.) DC.

Myrcianthes indifferens (McVaugh) McVaugh

Myrcianthes lindleyana (Kunth) McVaugh

Myrcianthes rhopaloides (Kunth) McVaugh

Myrteola nummularia (Poir.) O. Berg

Myrteola phylicoides (Benth.) L.R. Landrum

Psidium guajava $\mathrm{L}$.

Psidium guineense $\mathrm{Sw}$.

Psidium rutidocarpum Ruiz \& Pav.

Siphoneugena dussii (Krug \& Urb.) Proença

\section{Nyctaginaceae}

Boerhavia coccinea Mill.

Boerhavia diffusa $\mathrm{L}$.

Boerhavia erecta L.

Colignonia parviflora (Kunth) Choisy

Colignonia rufopilosa Kuntze

Commicarpus tuberosus (Lam.) Standl.

Mirabilis viscosa Cav.

Neea divaricata Poepp. \& Endl.

Neea floribunda Poepp. \& Endl.

Neea laxa Poepp. \& Endl.

Neea macrophylla Poepp. \& Endl.

Neea parviflora Poepp. \& Endl.

Neea spruceana Heimerl

Neea subpubescens Heimerl

Neea verticillata Ruiz \& Pav.

Neea virens Poepp. ex Heimerl

\section{Ochnaceae}

Cespedesia spathulata (Ruiz \& Pav.) Planch.

Godoya obovata Ruiz \& Pav.

Ouratea megaphylla Sastre

Ouratea oleosa J.F. Macbr.

Ouratea wallnoeferiana Sastre

Ouratea williamsii J.F. Macbr.

Perissocarpa ondox B. Walln.

Quiina amazonica A.C. Sm.

Quiina cruegeriana Griseb.

Sauvagesia erecta L. 
Baccharis tricuneata (L. f.) Pers.

Badilloa procera (B.L. Rob.) R.M. King \& H. Rob. Badilloa sphagnophila (B.L. Rob.) R.M. King \& H.

Rob.

Barnadesia dombeyana Less.

Barnadesia jelskii Hieron.

Barnadesia macbridei Ferreyra

Bartlettina macromeris (B.L. Rob.) R.M. King \& H. Rob.

Belloa kunthiana (DC.) Anderb. \& S.E. Freire

Bidens andicola Kunth

Bidens exigua Sherff

Bidens monticola Poepp. \& Endl.

Bidens pilosa L.

Bidens rubifolia Kunth

Bidens triplinervia Kunth

Brickellia diffusa (Vahl) A. Gray

Cacosmia rugosa Kunth

Calea pinnatifida (R. Br.) Less.

Chaptalia cordata Hieron.

Chaptalia nutans (L.) Pol.

Chaptalia oblonga D. Don

Chevreulia acuminata Less.

Chromolaena connivens (Rusby) R.M. King \& H. Rob.

Rob.

Chromolaena extensa (Gardner) R.M. King \& H.

Chromolaena ivaefolia (L.) R.M.King \& H.Rob.

Chromolaena laevigata (Lam.) R.M. King \& H. Rob.

Chromolaena leptocephala (DC.) R.M. King \& H.

Rob.

Chromolaena odorata (L.) R.M. King \& H. Rob.

Chromolaena squalida (DC.) R.M. King \& H. Rob.

Chrysactinium amphothrix (S.F. Blake) H. Rob. \&

Brettell

Chrysactinium caulescens (Hieron.) H. Rob. \&

Brettell

Chuquiraga spinosa Less.

Clibadium divaricatum S.F. Blake

Clibadium eggersii Hieron.

Conyza bonariensis (L.) Cronquist

Conyza laevigata (Rich.) Pruski

Coreopsis integra S.F. Blake

Coreopsis macbridei Sherff

Coreopsis microlepis S.F. Blake \& Sherff

Coreopsis sherffii S.F. Blake

Cotula mexicana (DC.) Cabrera

Critonia morifolia (Mill.) R.M. King \& H. Rob.

Critoniopsis boliviana (Britton) $\mathrm{H}$. Rob.

Critoniopsis jelskii (Hieron.) H. Rob.

Critoniopsis peruviana (Cuatrec.) H. Rob.

Critoniopsis pycnantha (Benth.) H. Rob.
Olacaceae

Cathedra acuminata (Benth.) Miers

Heisteria acuminata (Bonpl.) Engl.

Heisteria nitida Engl.

Heisteria ovata Benth.

Heisteria scandens Ducke

Heisteria spruceana Engl.

Minquartia guianensis Aubl.

\section{Oleandraceae}

Oleandra articulata (Sw.) C. Presl

\section{Onagraceae}

Epilobium denticulatum Ruiz \& Pav.

Epilobium pedicellare C. Presl

Fuchsia abrupta I.M. Johnst.

Fuchsia apetala Ruiz \& Pav.

Fuchsia boliviana Carrière

Fuchsia ceracea P.E. Berry

Fuchsia corymbiflora Ruiz \& Pav.

Fuchsia decussata Ruiz \& Pav.

Fuchsia ferreyrae P.E. Berry

Fuchsia huanucoensis P.E. Berry

Fuchsia macropetala C. Presl

Fuchsia macrophylla I.M. Johnst.

Fuchsia mezae P.E. Berry \& Hermsen

Fuchsia ovalis Ruiz \& Pav.

Fuchsia simplicicaulis Ruiz \& Pav.

Ludwigia affinis (DC.) H. Hara

Ludwigia decurrens Walter

Ludwigia helminthorrhiza (Mart.) H. Hara

Ludwigia hyssopifolia (G. Don) Exell

Ludwigia latifolia (Benth.) H. Hara

Ludwigia octovalvis (Jacq.) P.H. Raven

Ludwigia peruviana (L.) $\mathrm{H}$. Hara

Oenothera multicaulis Ruiz \& Pav.

Oenothera pubescens Willd. ex Spreng.

Oenothera rosea L'HÃ@r. ex Aiton

Oenothera sandiana Hassk.

Oenothera versicolor Lehm.

\section{Ophioglossaceae}

Botrychium virginianum (L.) Sw.

Ophioglossum crotalophoroides Walter

Ophioglossum palmatum L.

\section{Opiliaceae}

Agonandra peruviana Hiepko

\section{Orchidaceae}


Cronquistianthus callacatensis (Hieron.) R.M. King \&

H. Rob.

Cronquistianthus desmophyllus (B.L. Rob.) R.M.

King \& H. Rob.

Cronquistianthus determinatus (B.L. Rob.) R.M. King

\& H. Rob.

Cronquistianthus macbridei R.M. King \& H. Rob.

Cronquistianthus marrubiifolius (Hieron.) R.M. King

\& H. Rob.

Cuatrecasanthus sandemanii (H. Rob. \& B. Kahn) H. Rob.

Cynara scolymus L.

Cyrtocymura scorpioides (Lam.) H. Rob.

Dendrophorbium goodspeedii (Cuatrec.) H. Beltrán

Dendrophorbium usgorense (Cuatrec.) C. Jeffrey

Diplostephium gnidioides S.F. Blake

Diplostephium gynoxyoides Cuatrec.

Diplostephium hippophae S.F. Blake

Diplostephium peruvianum Cuatrec.

Diplostephium pulchrum S.F. Blake

Diplostephium vermiculatum Cuatrec.

Dorobaea pimpinellifolia (Kunth) B. Nord.

Dyssodia remota S.F. Blake

Eirmocephala brachiata (Benth.) H. Rob.

Eirmocephala cainarachiensis (Hieron.) H.Rob.

Elephantopus mollis Kunth

Erato polymnioides DC.

Erechtites hieraciifolia (L.) Raf. ex DC.

Erechtites hieraciifolius (L.) Raf. ex DC.

Erechtites valerianifolia (Link ex Wolf) Less. ex DC.

Erigeron primulifolium (Lam.) Greuter

Ferreyranthus excelsus (Poepp.) H. Rob. \& Brettell

Ferreyranthus verbascifolius (Kunth) H. Rob. \&

Brettell

Flaveria bidentis (L.) Kuntze

Fleischmannia altihuanucana $\mathrm{H}$. Rob.

Fleischmannia cookii (B.L. Rob.) R.M. King \& H. Rob.

Fleischmannia ferreyrii R.M. King \& H. Rob.

Fleischmannia fragilis (B.L. Rob.) R.M. King \& H.

Rob.

Fleischmannia lithophila (B.L. Rob.) R.M. King \& H. Rob.

Fleischmannia marginata (Poepp.) R.M. King \& H.

Rob.

Rob.

Fleischmannia pycnocephala (Less.) R.M. King \& H. Rob.

Fleischmannia rhodotephra (B.L. Rob.) R.M. King \& H. Rob.

Flourensia angustifolia (DC.) S.F. Blake
Acianthera decurrens (Poepp. \& Endl.) Pridgeon \& M.W. Chase

Acianthera lojae (Schltr.) Luer

Acronia discoidea (Lindl.) Luer

Acronia linguifera (Lindl.) Luer

Acronia phyllocardioides (Schltr.) Luer

Ada ocanensis (Lindl.) N.H. Williams

Alaticaulia amplexa (Luer) Luer

Alaticaulia bicolor (Poepp. \& Endl.) Luer

Alaticaulia concinna (Königer) Luer

Alaticaulia empusa (Luer) Luer

Alaticaulia jarae (Luer) Luer

Alaticaulia kuhniorum (Luer) Luer

Alaticaulia phoenix (Luer) Luer

Alaticaulia pyxis (Luer) Luer

Altensteinia longispicata C. Schweinf.

Anacheilium bennettii (Christenson) Withner \& P.A.

Harding

Anathallis francesiana (Luer) Luer

Anguloa uniflora Ruiz \& Pav.

Aspidogyne foliosa (Poepp. \& Endl.) Garay

Benzingia hajekii (D.E. Benn. \& Christenson) Dressler

Benzingia jarae (D.E. Benn. \& Christenson) Dressler

Bletia catenulata Ruiz \& Pav.

Brachystele unilateralis (Poir.) Schltr.

Brassia caudata (L.) Lindl.

Brassia davidii Senghas

Brassia koehlerorum Schltr.

Brassia neglecta Rchb. f.

Brassia peruviana Poepp. \& Endl.

Brassia wageneri Rchb. f.

Bulbophyllum incarum Kraenzl.

Byrsella anomala (Luer \& Sijm) Luer

Camaridium vestitum (Sw.) Lindl.

Catasetum cotylicheilum D.E. Benn. \& Christenson

Catasetum jarae Dodson \& D.E. Benn.

Catasetum monzonensis D.E. Benn. \& Christenson

Catasetum nanayanum Dodson \& D.E. Benn.

Catasetum schweinfurthii D.E. Benn. \& Christenson

Catasetum transversicallosum D.E. Benn. \&

Christenson

Chaubardia klugii (C. Schweinf.) Garay 
Galinsoga parviflora Cav.

Galinsoga quadriradiata Ruiz \& Pav.

Gamochaeta americana (Mill.) Wedd.

Gamochaeta purpurea (L.) Cabrera

Gnaphalium dombeyanum DC.

Gnaphalium polium Wedd.

Gnaphalium pulchrum Steud.

Gynoxys buxifolia (Kunth) Cass.

Gynoxys capituliparva Cuatrec.

Gynoxys caracensis Muschl.

Gynoxys compressissima Cuatrec.

Gynoxys congestiflora Sagást. \& M.O. Dillon

Gynoxys huasahuasis Cuatrec.

Gynoxys macfrancisci Cuatrec.

Gynoxys macrophylla Muschl.

Gynoxys monzonensis Mattf.

Gynoxys vacana Cuatrec.

Gynoxys woytkowskii (Cuatrec.) Cuatrec.

Hebeclinium beneolens (B.L. Rob.) R.M. King \& H.

Rob.

Hebeclinium bullatissimum (B.L. Rob.) R.M. King \&

H. Rob.

Hebeclinium macrophyllum (L.) DC.

Hebeclinium vestitum R.M. King \& H. Rob.

Heliopsis buphthalmoides (Jacq.) Dunal

Heterocondylus vitalbae (DC.) R.M. King \& H. Rob.

Heterosperma diversifolium Kunth

Hieracium peruanum Fr.

Hypochaeris taraxacoides Ball

Ichthyothere scandens S.F. Blake

Jaegeria hirta (Lag.) Less.

Jungia axillaris (Lag. ex DC.) Spreng.

Jungia floribunda Less.

Jungia paniculata (DC.) A. Gray

Jungia vitocensis Cuatrec.

Jungia weberbaueri Cerrate

Koanophyllon celtidifolia (Lam.) R.M.King \& H.Rob.

Koanophyllon isillumense (B.L. Rob.) R.M. King \& H. Rob.

Koanophyllon solidaginoides (Kunth) R.M. King \& H. Rob.

Laennecia gnaphalioides (Kunth) Cass.

Lasiocephalus patens (Kunth) Cuatrec.

Lepidaploa canescens (Kunth) H. Rob.

Lepidaploa myriocephala (DC.) H. Rob.

Lepidaploa salzmannii (DC.) H. Rob.

Liabum amplexicaule Poepp.

Liabum eriocaulon Poepp.

Liabum ferreyrii $\mathrm{H}$. Rob.
Chaubardiella hirtzii Dodson

Chondroscaphe flaveola (Linden \& Rchb. f. ex Rchb.

f.) Senghas \& G. Gerlach

Cischweinfia jarae Dodson \& D.E. Benn.

Cischweinfia parva (C. Schweinf.) Dressler \& N.H.

Williams

Cranichis multiflora (Poepp. \& Endl.) Cogn.

Cryptocentrum inaequisepalum C. Schweinf.

Cyclopogon inaequilaterus (Poepp. \& Endl.) Schltr.

Cyclopogon ovalifolium C. Presl

Cycnoches christensonii D.E. Benn.

Cycnoches egertonianum Bateman

Cycnoches haagii Barb. Rodr.

Cycnoches jarae Dodson \& D.E. Benn.

Cyrtochilum aureum (Lindl.) Senghas

Cyrtochilum cimiciferum (Rchb. f.) Dalström

Cyrtochilum cordatum (Lindl.) Kraenzl.

Cyrtochilum flexuosum Kunth

Cyrtochilum hastatum (Ruiz \& Pav.) Mansf. ex

Dalström

Cyrtochilum insculptum (Rchb. f.) Kraenzl.

Cyrtochilum ligulatum (Ruiz \& Pav.) Mansf. ex

Dalström

Cyrtochilum myanthum (Lindl.) Kraenzl.

Cyrtochilum tricostatum Kraenzl.

Cyrtochilum volubile Poepp. \& Endl.

Dichaea calyculata Poepp. \& Endl.

Dichaea laxa (Ruiz \& Pav.) Poepp. \& Endl.

Dichaea morrisii Fawc. \& Rendle

Dracula deniseana Luer

Dressleria aurorae H.G. Hills \& D.E. Benn.

Elleanthus aurantiacus (Lindl.) Rchb. $\mathrm{f}$.

Elleanthus capitatus (Poepp. \& Endl.) Rchb. f.

Elleanthus conifer (Rchb. f. \& Warsz.) Rchb. f.

Elleanthus fractiflexus Schltr.

Elleanthus furfuraceus (Lindl.) Rchb. f.

Elleanthus graminifolius (Barb. Rodr.) Løjtnant

Elleanthus oliganthus (Poepp. \& Endl.) Rchb. f.

Elleanthus strobilifer (Poepp. \& Endl.) Rchb. f.

Elleanthus wallnoeferi Szlach. ex Szlach.

Elongatia sijmii (Luer) Luer

Encyclia sclerocladia (Lindl. ex Rchb. f.) Hoehne Epidendrum albifloroides D.E. Benn. \& Christenson Epidendrum ampliracemum C. Schweinf.

Epidendrum anceps Jacq.

Epidendrum apaganoides D.E. Benn. \& Christenson

Epidendrum azulensis D.E. Benn. \& Christenson

Epidendrum bennettii Dodson

Epidendrum bicirrhatum D.E. Benn. \& Christenson 
Liabum macbridei $\mathrm{H}$. Rob.

Liabum nudicaule H. Rob.

Liabum solidagineum (Kunth) Less.

Lophopappus peruvianus Cabrera

Loricaria lucida Cuatrec.

Loricaria thuyoides (Lam.) Sch. Bip.

Luciliocline subspicata (Wedd.) Anderb. \& S.E.

Freire

Lycoseris trinervis (D. Don) S.F. Blake

Mikania angularis Bonpl.

Mikania aquaria B.L. Rob.

Mikania aschersonii Hieron.

Mikania banisteriae DC.

Mikania conglomerata W.C. Holmes \& McDaniel

Mikania cordifolia (L. f.) Willd.

Mikania cuencana Hieron.

Mikania cutervensis Hieron.

Mikania decora Poepp.

Mikania dioscoreoides (Rusby) B.L. Rob.

Mikania featherstonei B.L. Rob.

Mikania glandulifera W.C. Holmes \& McDaniel

Mikania guaco Bonpl.

Mikania haenkeana DC.

Mikania hookeriana DC.

Mikania huanucoensis W.C. Holmes \& McDaniel

Mikania incasina B.L. Rob.

Mikania jelskii Hieron.

Mikania leiostachya Benth.

Mikania macdanielii W.C. Holmes

Mikania macrostipulata W.C. Holmes \& McDaniel

Mikania megalophylla W.C. Holmes \& McDaniel

Mikania micrantha Kunth

Mikania montana B.L. Rob.

Mikania nemorosa Klatt

Mikania oreopola B.L. Rob.

Mikania parvicapitulata Hieron.

Mikania psilostachya DC.

Mikania stuebelii Hieron.

Mikania szyszylowiczii Hieron.

Mikania ternata (Vell.) B.L. Rob.

Mikania trichodes B.L.Rob

Mikania vitifolia DC.

Mikania weberbaueri Hieron.

Munnozia affinis (S.F. Blake) H. Rob. \& Brettell

Munnozia corymbosa Ruiz \& Pav.

Munnozia hastifolia (Poepp.) H. Rob. \& Brettell

Munnozia lanceolata Ruiz \& Pav.

Munnozia oxyphylla (Cuatrec.) H. Rob. \& Brettell
Epidendrum bidens D.E. Benn. \& Christenson

Epidendrum calanthum Rchb. f. \& Warsz.

Epidendrum calyptratoides Hágsater \& Dodson

Epidendrum capitellatum C. Schweinf.

Epidendrum compressibulbum D.E. Benn. \&

Christenson

Epidendrum difforme Jacq.

Epidendrum elatum C. Schweinf.

Epidendrum forcipatum C. Schweinf.

Epidendrum fritzianum Hoehne

Epidendrum fujimorianum D.E. Benn. \& Christenson

Epidendrum guacamayense Hágsater \& Dodson

Epidendrum haenkeanum $\mathrm{C}$. Presl

Epidendrum jajense Rchb. f.

Epidendrum jarae D.E. Benn. \& Christenson

Epidendrum kanehirae Hágsater

Epidendrum liguliferum C. Schweinf.

Epidendrum macrocyphum Kraenzl.

Epidendrum melanoporphyreum Hágsater

Epidendrum miradoranum Dodson \& D.E. Benn.

Epidendrum modestum Rchb. f. \& Warsz.

Epidendrum monzonense Kraenzl.

Epidendrum nocturnum Jacq.

Epidendrum paniculatum Ruiz \& Pav.

Epidendrum parviflorum Ruiz \& Pav.

Epidendrum platyoon Schltr.

Epidendrum schizoclinandrium D.E. Benn. \&

Christenson

Epidendrum secundum Jacq.

Epidendrum strictiforme C. Schweinf.

Epidendrum subreniforme C. Schweinf.

Epidendrum tingo-mariae Hágsater

Epidendrum tricarinatum Rolfe

Epidendrum uncinatum D.E. Benn. \& Christenson

Epidendrum viride Ruiz \& Pav.

Epidendrum weberbauerianum Kraenzl.

Epidendrum whittenii Hágsater \& Dodson

Epidendrum woytkowskianum A.D. Hawkes

Epistephium amplexicaule Poepp. \& Endl.

Erycina glossomystax (Rchb. f.) N.H. Williams \& M.W.

Chase

Erycina pusilla (L.) N.H. Williams \& M.W. Chase

Erythrodes major (C. Presl) Ames

Erythrodes minor (C. Presl) Ames

Exalaria parviflora Garay \& G.A. Romero-Gonzalez

Fernandezia distichoides M.W. Chase

Fernandezia ionanthera (Rchb. f. \& Warsz.) Schltr.

Fernandezia lycopodioides (Schltr.) M.W. Chase

Fernandezia parviflora (Lindl.) M.W. Chase

Fernandezia pectinata (Rchb. f.) Rchb. f. 
Munnozia peruensis (Cuatrec.) H. Rob. \& Brettell Munnozia senecionidis Benth.

Munnozia silphioides (Poepp.) H. Rob. \& Brettell Munnozia venosissima Ruiz \& Pav.

Mutisia acuminata Ruiz \& Pav.

Mutisia cochabambensis Hieron.

Mutisia lanata Ruiz \& Pav.

Nordenstamia huanucona (Cuatrec.) B. Nord.

Noticastrum macbridei Cuatrec.

Novenia tunariensis (Kuntze) S.E. Freire

Onoseris albicans (D. Don) Ferreyra

Ophryosporus apricus B.L. Rob.

Ophryosporus chilca (Kunth) Hieron.

Ophryosporus macbridei B.L. Rob.

Ophryosporus piquerioides (DC.) Benth. ex Baker

Oritrophium limnophilum (Sch. Bip.) Cuatrec.

Orthopappus angustifolius (Sw.) Gleason

Oyedaea buphthalmoides DC.

Pappobolus acuminatus (S.F. Blake) Panero

Pappobolus discolor (S.F. Blake) Panero

Pappobolus macranthus S.F. Blake

Pappobolus senex (S.F. Blake) Panero

Paranephelius bullatus A. Gray ex Wedd.

Paranephelius ovatus A. Gray ex Wedd.

Pentacalia asplundii (Cabrera) Cuatrec.

Pentacalia bacopoides (Greenm. \& Cuatrec.)

Cuatrec.

Pentacalia brittoniana (Hieron.) Cuatrec.

Pentacalia carpishensis (Cuatrec.) Cuatrec.

Pentacalia divisoria (Cabrera) Cuatrec.

Pentacalia epiphytica (Kuntze) Cuatrec.

Pentacalia huamaliensis (Cabrera) Cuatrec.

Pentacalia lucidissima (Cuatrec.) Cuatrec.

Pentacalia oronocensis (DC.) Cuatrec.

Pentacalia reflexa (Kunth) Cuatrec.

Perezia multiflora (Bonpl.) Less.

Perezia pinnatifida (Bonpl.) Wedd.

Perezia pungens (Bonpl.) Less.

Picrosia longifolia D. Don

Piptocarpha asterotrichia (Poepp.) Baker

Piptocarpha opaca (Benth.) Baker

Piptocarpha poeppigiana (DC.) Baker

Plagiocheilus solivaeformis DC.

Polyanthina nemorosa (Klatt) R.M. King \& H. Rob.

Porophyllum ruderale (Jacq.) Cass.

Praxelis clematidea R.M. King \& H. Rob.

Pseudelephantopus spiralis (Less.) Cronquist

Pseudobaccharis inamoena (Gardner) Teodoro

Quechualia fulta (Griseb.) H. Rob.
Gomphichis goodyeroides Lindl.

Gomphichis macbridei C. Schweinf.

Habenaria floribunda Lindl.

Habenaria monorrhiza (Sw.) Rchb. f.

Habenaria parvidens Lindl.

Habenaria rupestris Poepp. \& Endl.

Heterotaxis equitans (Schltr.) Ojeda \& Carnevali

Houlletia sanderi Rolfe

Ida ciliata (Ruiz \& Pav.) A. Ryan \& Oakeley

Ida diastasia (D.E. Benn. \& Oakeley) A. Ryan \&

Oakeley

Ida reichenbachii (Gireoud ex Rchb. f.) A. Ryan \&

Oakeley

lonopsis satyrioides (Sw.) Rchb. f.

Isochilus linearis (Jacq.) R. Br.

Jacquiniella globosa (Jacq.) Schltr.

Kefersteinia bertoldii Jenny

Kefersteinia jarae D.E. Benn. \& Christenson

Kefersteinia sanguinolenta Rchb. f.

Lepanthes adrianae Luer

Lepanthes applanata Luer \& Sijm

Lepanthes aurorae D.E. Benn. \& Christenson

Lepanthes bivalvis Luer \& Sijm

Lepanthes capistrata Luer \& Sijm

Lepanthes caprimulgus Luer

Lepanthes caudatisepala C. Schweinf.

Lepanthes cloesii Luer

Lepanthes echinata Luer \& Cloes

Lepanthes erucifera Luer \& Sijm

Lepanthes laxa Luer \& J. Portilla

Lepanthes martineae Luer \& Cloes

Lepanthes pumila C. Schweinf.

Lepanthes revoluta Luer \& Cloes

Lepanthopsis acetabulum Luer

Liparis ramosa Poepp. \& Endl.

Lockhartia amoena Endrés \& Rchb. f.

Lockhartia lepticaula D.E. Benn. \& Christenson

Lophiaris nana (Lindl.) Braem

Luzama amaluzae (Luer \& Malo) Luer

Luzama pyknosepala (Luer \& Cloes) Luer

Lycaste mathiasiae G.C. Kenn.

Lycomormium squalidum (Poepp. \& Endl.) Rchb. f.

Macroclinium biflorum D.E. Benn. \& Christenson

Macroclinium chasei Dodson \& D.E. Benn.

Malaxis andicola (Ridl.) Kuntze

Malaxis excavata (Lindl.) Kuntze

Malaxis hieronymi (Cogn.) L.O. Williams

Malaxis ventricosa (Poepp. \& Endl.) Kuntze

Masdevallia calosiphon Luer

Masdevallia carpishica Luer \& Cloes 
Rhysolepis helianthoides (Rich.) H. Rob. \& A.J.

Moore

Schistocarpha eupatorioides (Fenzl) Kuntze

Schistocarpha sinforosi Cuatrec.

Schkuhria pinnata (Lam.) Kuntze ex Thell.

Senecio axillifoliatus Cuatrec.

Senecio bolivarianus Cuatrec.

Senecio breviscapus DC.

Senecio burkartii Cabrera

Senecio canescens (Bonpl.) Cuatrec.

Senecio chavanilloensis Cuatrec.

Senecio collinus DC.

Senecio comosus Sch. Bip.

Senecio condimentarius Cabrera

Senecio evacoides Sch. Bip.

Senecio featherstonii Cuatrec.

Senecio ferreyrae Cabrera

Senecio haenkeanus Cuatrec.

Senecio jungioides Cabrera

Senecio killipii Cabrera

Senecio lancidentatus Cuatrec.

Senecio macrorrhizus Wedd.

Senecio melanocalyx Cuatrec.

Senecio minesinus Cuatrec.

Senecio mitonis Cuatrec.

Senecio nutans Sch. Bip.

Senecio puna-sessilis Cuatrec.

Senecio rhizomatus Rusby

Senecio serratifolius (Meyen \& Walp.) Cuatrec.

Senecio spinosus DC.

Senecio tenuisagittatus Cuatrec.

Senecio torrehuasensis Cuatrec.

Smallanthus fruticosus (Benth.) H. Rob.

Smallanthus glabratus (DC.) H. Rob.

Smallanthus sonchifolius (Poepp.) H. Rob.

Stevia leucosticta B.L. Rob.

Stevia macbridei B.L. Rob.

Stevia ovata Willd.

Stevia pearcei B.L. Rob.

Stevia puberula Hook.

Struchium sparganophorum (L.) Kuntze

Stuckertiella capitata (Wedd.) Beauverd

\begin{tabular}{|l|}
\hline Tagetes dianthiflora Kunth \\
\hline Tagetes elliptica Sm. \\
\hline Tagetes terniflora Kunth \\
\hline Tanacetum parthenium (L.) Sch. Bip. \\
\hline Tessaria integrifolia Ruiz \& Pav. \\
\hline Trepadonia mexiae (H. Rob.) H. Rob. \\
\hline Trichocline hieracioides (Kunth) Ferreyra \\
\hline
\end{tabular}

Masdevallia constricta Poepp. \& Endl.

Masdevallia fuchsii Luer

Masdevallia harlequina Luer

Masdevallia immensa Luer

Masdevallia lilacina Königer

Masdevallia manoloi Luer \& M. Arias

Masdevallia pandurilabia C. Schweinf.

Masdevallia prolixa Luer

Masdevallia pumila Poepp. \& Endl.

Masdevallia rodolfoi (Braas) Luer

Maxillaria argyrophylla Poepp. \& Endl.

Maxillaria aurea (Poepp. \& Endl.) L.O. Williams

Maxillaria batemanii Poepp. \& Endl.

Maxillaria bennettii Christenson

Maxillaria breviscapa Poepp. \& Endl.

Maxillaria callichroma Rchb. f.

Maxillaria edwardsii D.E. Benn. \& Christenson

Maxillaria exaltata (Kraenzl.) C. Schweinf.

Maxillaria frechettei D.E. Benn. \& Christenson

Maxillaria graminifolia (Kunth) Rchb. $\mathrm{f}$.

Maxillaria haemathodes (Ruiz \& Pav.) Garay

Maxillaria huanucoensis D.E. Benn. \& Christenson

Maxillaria lindleyana Schltr.

Maxillaria platypetala Ruiz \& Pav.

Maxillaria prolifera Ruiz \& Pav.

Maxillaria ramosissima Kraenzl.

Maxillaria splendens Poepp. \& Endl.

Maxillaria trilobulata D.E. Benn. \& Christenson

Maxillaria virguncula Rchb. $\mathrm{f}$.

Maxillariella graminifolia (Kunth) M.A. Blanco \&

Carnevali

Mesadenella peruviana Garay

Miltoniopsis bismarkii Dodson \& D.E. Benn.

Mormodes andicola Salazar

Mormolyca richii (Dodson) M.A. Blanco

Mormolyca rufescens (Lindl.) M.A. Blanco

Myoxanthus affinis (Lindl.) Luer

Myoxanthus exasperatus (Lindl.) Luer

Myoxanthus monophyllus Poepp. \& Endl.

Myoxanthus serripetalus (Kraenzl.) Luer

Myrosmodes paludosum (Rchb. f.) P. Ortiz

Neobennettia genegeorgei (D.E. Benn. \&

Christenson) Senghas

Neodryas rhodoneura Rchb. f.

Octomeria scirpoidea (Poepp. \& Endl.) Rchb. f.

Odontoglossum epidendroides Kunth

Odontoglossum gracile Lindl.

Oncidium buchtienoides M.W. Chase \& N.H. Williams

Oncidium fasciferum Rchb. f. \& Warsz.

Oncidium fuscatum Rchb. f. 
Tridax procumbens $\mathrm{L}$.

Verbesina grandifolia S.F. Blake

Verbesina grandis S.F. Blake

Verbesina macbridei S.F. Blake

Verbesina malacophylla S.F. Blake

Verbesina pauciramea Sagást., S. Leiva \& Lezama

Vernonia patens Kunth

Viguiera macbridei S.F. Blake

Werneria amblydactyla S.F. Blake

Werneria nubigena Kunth

Werneria pygmaea Gillies ex Hook. \& Arn.

Xanthium spinosum L.

Xenophyllum dactylophyllum (Sch. Bip.) V.A. Funk

Zinnia peruviana (L.) L.

\section{Athyriaceae}

Diplazium grandifolium (Sw.) Sw.

Diplazium pinnatifidum Kunze

Diplazium trianae (Mett.) C. Chr.

Diplazium tungurahuae (Sodiro) C. Chr.

\section{Balanophoraceae}

Langsdorffia hypogaea Mart.

Ombrophytum peruvianum Poepp. \& Endl.

\section{Balsaminaceae}

Impatiens balsamina $\mathrm{L}$.

\section{Basellaceae}

Anredera cordifolia (Ten.) Steenis

Ullucus tuberosus Caldas

\section{Begoniaceae}

Begonia bracteosa A. DC.

Begonia cyathophora Poepp. \& Endl.

Begonia foliosa Kunth

Begonia glabra Aubl.

Begonia glauca (Klotzsch) Ruiz \& Pav. ex A. DC.

Begonia glaucoides Irmsch.

Begonia hirta (Klotzsch) L.B. Sm. \& B.G. Schub.

Begonia humilis Dryand.

Begonia lophoptera Rolfe

Begonia maynensis A. DC.

Begonia monadelpha (Klotzsch) Ruiz \& Pav. ex A. DC.

Begonia obtecticaulis Irmsch.

Begonia octopetala L'HÃ@r.

Begonia parviflora Poepp. \& Endl.

Begonia peruviana A. DC.

Begonia prionophylla Irmsch.

Begonia pseudoglauca Irmsch.

Begonia rossmanniae A. DC.
Oncidium gramineum (Poepp. \& Endl.) M.W. Chase \&

N.H. Williams

Oncidium retusum Lindl.

Oncidium tigroides (C. Schweinf.) M.W. Chase \& N.H.

Williams

Oncidium trilingue Lindl. \& Paxton

Oncidium wyattianum (A.G. Wilson) M.W. Chase \&

N.H. Williams

Ornithidium nubigenum Rchb. f.

Ornithidium pseudonubigenum (J.T. Atwood) M.A.

Blanco \& Ojeda

Ornithocephalus ciliatus Lindl.

Pelexia pavonii (Rchb. f.) Garay

Phragmipedium boissierianum (Rchb. f.) Rolfe

Phragmipedium caudatum (Lindl.) Rolfe

Pleurothallis flexuosa (Poepp. \& Endl.) Lindl.

Pleurothallis fonsflorum Lindl.

Pleurothallis furcifera Luer

Pleurothallis loranthophylla Rchb. f.

Pleurothallis mexiae Luer

Pleurothallis ruscifolia (Jacq.) R. Br.

Pleurothallis talpinaria Rchb. $\mathrm{f}$.

Pleurothallis tridentata Klotzsch

Pleurothallis undulata Poepp. \& Endl.

Polycycnis silvana F. Barros

Polystachya foliosa (Hook.) Rchb. f.

Ponthieva fertilis (F. Lehm. \& Kraenzl.) Salazar

Ponthieva inaudita Rchb. $\mathrm{f}$.

Ponthieva oligoneura Schltr.

Ponthieva pubescens (C. Presl) C. Schweinf.

Ponthieva similis C. Schweinf.

Ponthieva weberbaueri Schltr.

Porroglossum meridionale P. Ortiz

Prosthechea aemula (Lindl.) W.E. Higgins

Prosthechea grammatoglossa (Rchb. f.) W.E. Higgins

Prosthechea pulchra Dodson \& W.E. Higgins

Prosthechea vespa (Vell.) W.E. Higgins

Psychopsis sanderae (Rolfe) Lückel \& Braem

Restrepia antennifera Kunth

Rodriguezia satipoana Dodson \& D.E. Benn.

Ronaldella aryter (Luer) Luer

Rusbyella caespitosa Rolfe

Sarcoglottis acaulis (Sm.) Schltr.

Sauroglossum schweinfurthianum Garay

Scaphosepalum antenniferum Rolfe

Scaphyglottis boliviensis (Rolfe) B.R. Adams

Scaphyglottis graminifolia (Ruiz \& Pav.) Poepp. \&

Endl.

Scaphyglottis leucantha Rchb. f.

Scaphyglottis micrantha (Lindl.) Ames \& Correll

Scaphyglottis prolifera (Sw.) Cogn. 
Begonia urticae L. f.

Begonia viridiflora A. DC.

\section{Berberidaceae}

Berberis benoistiana J.F. Macbr.

Berberis brumalis J.F. Macbr.

Berberis carinata Lechl.

Berberis commutata Eichler

Berberis flexuosa Ruiz \& Pav.

Berberis hochreutinerana J.F. Macbr.

Berberis jelskiana C.K. Schneid.

Berberis latifolia Ruiz \& Pav.

Berberis lutea Ruiz \& Pav.

Berberis multiflora Benth.

Berberis tomentosa Ruiz \& Pav.

\section{Betulaceae}

Alnus acuminata Kunth

Alnus jorullensis Kunth

\section{Bignoniaceae}

Adenocalymma cladotrichum (Sandwith) L.G.

Lohmann

Adenocalymma impressum (Rusby) Sandwith

Adenocalymma purpurascens Rusby

Adenocalymma uleanum Kraenzl.

Amphilophium crucigerum (L.) L.G. Lohmann

Amphilophium paniculatum (L.) Kunth

Bignonia binata Thunb.

Bignonia bracteomana (K. Schum. ex Sprague) L.G.

Lohmann

Bignonia lilacina (A.H. Gentry) L.G. Lohmann

Bignonia nocturna (Barb. Rodr.) L.G. Lohmann

Bignonia uleana (Kraenzl.) L.G. Lohmann

Callichlamys latifolia (Rich.) K. Schum.

Cuspidaria floribunda (DC.) A.H. Gentry

Delostoma integrifolium D. Don

Dolichandra uncata (Andrews) L.G. Lohmann

Eccremocarpus viridis Ruiz \& Pav.

Fridericia florida (DC.) L.G. Lohmann

Fridericia patellifera (Schltdl.) L.G. Lohmann

Fridericia pearcei (Rusby) L.G. Lohmann

Fridericia platyphylla (Cham.) L.G. Lohmann

Fridericia schumanniana (Loes.) L.G. Lohmann

Handroanthus chrysanthus (Jacq.) S.O. Grose

Handroanthus impetiginosus (Mart. ex DC.) Mattos

Handroanthus ochraceus (Cham.) Mattos

Handroanthus serratifolius (Vahl) S.O. Grose

Jacaranda acutifolia Bonpl.

Jacaranda copaia (Aubl.) D. Don

Jacaranda glabra (A. DC.) Bureau \& K. Schum.

Lundia spruceana Bureau

Mansoa alliacea (Lam.) A.H. Gentry
Scaphyglottis punctulata (Rchb. f.) C. Schweinf.

Scaphyglottis summersii L.O. Williams

Sobralia caloglossa Schltr.

Sobralia dichotoma Ruiz \& Pav.

Sobralia fimbriata Poepp. \& Endl.

Sobralia klotzscheana Rchb. f.

Sobralia rosea Poepp. \& Endl.

Sobralia setigera Poepp. \& Endl.

Sobralia valida Rolfe

Stanhopea pozoi Dodson \& D.E. Benn.

Stelis biserrula Lindl.

Stelis capillaris Lindl.

Stelis diffusa C. Schweinf.

Stelis gemma Garay

Stelis intermedia Poepp. \& Endl.

Stelis lindenii Lindl.

Stelis purpurea (Ruiz \& Pav.) Willd.

Stelis sijmii Luer

Stelis superbiens Lindl.

Stelis uninervia C. Schweinf.

Stelis xiphizusa (Rchb. f.) Pridgeon \& M.W. Chase

Stenia guttata Rchb. f.

Stenia jarae D.E. Benn.

Stenia pallida Lindl.

Stenoptera laxiflora C. Schweinf.

Stenoptera montana C. Schweinf.

Stenoptera peruviana C. Presl

Sudamerlycaste cobbiana (B.S. Williams) Archila

Sudamerlycaste dyeriana (Sander ex Rolfe) Archila

Talpinaria hitchcockii (Ames) Luer

Telipogon bennettii (Dodson \& R. Escobar) N.H.

Williams \& Dressler

Telipogon intis Braas

Telipogon lueri Dodson \& D.E. Benn.

Telipogon urceolatus C. Schweinf.

Trichopilia fragrans (Lindl.) Rchb. f.

Trichosalpinx quitensis (Rchb. f.) Luer

Trisetella cordeliae Luer

Vanilla hamata Klotzsch

Vanilla ruiziana Klotzsch

Xerorchis trichorhiza (Kraenzl.) Garay

Xylobium pallidiflorum (Hook.) G. Nicholson

Xylobium squalens (Lindl.) Lindl.

Xylobium subpulchrum Dressler

Xylobium undulatum (Ruiz \& Pav.) Rolfe

Zootrophion beloglottis (Schltr.) Luer

Zygostates apiculata (Lindl.) Toscano

\section{Orobanchaceae}

Agalinis pennellii Barringer 
Mansoa standleyi (Steyerm.) A.H. Gentry Martinella obovata (Kunth) Bureau \& K. Schum. Stizophyllum inaequilaterum Bureau \& K. Schum. Stizophyllum riparium (Kunth) Sandwith Tanaecium pyramidatum (Rich.) L.G. Lohmann Tecoma stans (L.) Juss. ex Kunth Tynanthus polyanthus (Bureau) Sandwith Tynanthus villosus A.H. Gentry Xylophragma platyphyllum (DC.) L.G. Lohmann Xylophragma pratense (Bureau \& K. Schum.)

Sprague

\section{Bixaceae}

Bixa orellana L.

Bixa platycarpa Ruiz \& Pav. ex G. Don

\section{Blechnaceae}

Blechnum asplenioides Sw.

\section{Boraginaceae}

Cordia alliodora (Ruiz \& Pav.) Oken

Cordia lippioides I.M. Johnst.

Cordia lomatoloba I.M. Johnst.

Cordia lutea Lam.

Cordia nodosa Lam.

Cordia panamensis L. Riley

Cordia scaberrima Kunth

Cordia ucayaliensis (I.M. Johnst.) I.M. Johnst.

Heliotropium incanum Ruiz \& Pav.

Heliotropium indicum L.

Heliotropium mandonii I.M. Johnst.

Heliotropium microstachyum Ruiz \& Pav.

Heliotropium submolle Klotzsch

Lithospermum macbridei I.M. Johnst.

Nama dichotoma (Ruiz \& Pav.) Choisy

Phacelia secunda J.F. Gmel.

Plagiobothrys myosotoides (Lehm.) Brand

Tournefortia angustiflora Ruiz \& Pav.

Tournefortia chinchensis Killip

Tournefortia cuspidata Kunth

Tournefortia fuliginosa Kunth

Tournefortia hirsutissima L.

Tournefortia lilloi I.M. Johnst.

Tournefortia longifolia Ruiz \& Pav.

Tournefortia maculata Jacq.

Tournefortia microcalyx (Ruiz \& Pav.) I.M. Johnst.

Tournefortia ovalifolia Rusby

Tournefortia polystachya Ruiz \& Pav.

Tournefortia setacea Killip

Tournefortia virgata Ruiz \& Pav.

Varronia crenata Ruiz \& Pav.

Varronia curassavica Jacq.

Varronia dichotoma Ruiz \& Pav.
Bartsia aprica Diels

Bartsia canescens Wedd.

Bartsia crisafullii N.H. Holmgren

Bartsia elachophylla Diels

Bartsia filiformis Wedd.

Bartsia inaequalis Benth.

Bartsia melampyroides (Kunth) Benth.

Bartsia patens Benth.

Bartsia pedicularoides Benth.

Bartsia pyricarpa Molau

Bartsia trichophylla Wedd.

Bartsia weberbaueri Diels

Castilleja cerroana Edwin

Castilleja profunda T.I. Chuang \& Heckard

Castilleja pumila (Benth.) Wedd.

Castilleja scorzonerifolia Kunth

\section{Oxalidaceae}

Biophytum amazonicum R. Knuth

Biophytum peruvianum R. Knuth

Biophytum soukupii Lourteig

Biophytum tessmannii R. Knuth

Oxalis boliviana Britton

Oxalis caucensis R. Knuth

Oxalis cinerea Zucc.

Oxalis corniculata L.

Oxalis dolichopoda Diels

Oxalis humbertii Lourteig

Oxalis integra R. Knuth

Oxalis latifolia Kunth

Oxalis lespedezioides G. Don

Oxalis lucumayensis R. Knuth

Oxalis macbridei R. Knuth

Oxalis megalorrhiza Jacq.

Oxalis melilotoides Zucc.

Oxalis mollis Kunth

Oxalis mollissima (Rusby) R. Knuth

Oxalis nubigena Walp.

Oxalis ortgiesii Regel

Oxalis pachyrrhiza Wedd.

Oxalis peduncularis Kunth

Oxalis pickeringii A. Gray

Oxalis salticola Lourteig

Oxalis sanmiguelii R. Knuth

Oxalis spiralis Ruiz \& Pav. ex G. Don

Oxalis spruceana Progel

Oxalis tabaconasensis R. Knuth

Oxalis tessmannii R. Knuth

Oxalis tuberosa Molina

Papaveraceae 
Varronia spinescens (L.) Borhidi

\section{Brassicaceae}

Brassica oleracea L.

Cardamine bonariensis Pers.

Cardamine ovata Benth.

Descurainia athrocarpa (A. Gray) O.E. Schulz

Descurainia depressa (Phil.) Prantl ex Reiche

Descurainia myriophylla (Willd. ex DC.) R.E. Fr.

Draba peruviana (DC.) O.E. Schulz

Lepidium bipinnatifidum Desv.

Raphanus raphanistrum L.

Rorippa nana (Schltdl.) J.F. Macbr.

Weberbauera scabrifolia Al-Shehbaz

Weberbauera spathulifolia (A. Gray) O.E. Schulz

\section{Bromeliaceae}

Aechmea angustifolia Poepp. \& Endl.

Aechmea bromeliifolia (Rudge) Baker

Aechmea longifolia (Rudge) L.B. Sm. \& M.A.

Spencer

Aechmea paniculata Ruiz \& Pav.

Aechmea vallerandii (Carrière) Erhardt, Götz \&

Seybold

Aechmea veitchii Baker

Ananas comosus (L.) Merr.

Ananas lucidus Mill.

Billbergia incarnata (Ruiz \& Pav.) Schult. f.

Bromelia tarapotina Ule

Catopsis sessiliflora (Ruiz \& Pav.) Mez

Fosterella penduliflora (C.H. Wright) L.B. Sm.

Fosterella schidosperma (Baker) L.B. Sm.

Greigia macbrideana L.B. Sm.

Guzmania apiculata L.B. Sm.

Guzmania brevispatha Mez

Guzmania devansayana E. Morren

Guzmania dudleyi L.B. Sm.

Guzmania globosa L.B. Sm.

Guzmania marantoidea (Rusby) H. Luther

Guzmania monostachia (L.) Rusby ex Mez

Guzmania retusa L.B. Sm.

Guzmania squarrosa (Mez \& Sodiro) L.B. Sm. \&

Pittendr.

Guzmania strobilantha (Ruiz \& Pav.) Mez

Guzmania tarapotina Ule

Mezobromelia capituligera (Griseb.) J.R. Grant

Neoregelia eleutheropetala (Ule) L.B. Sm.

Pitcairnia asplundii L.B. Sm.

Pitcairnia bifaria L.B. Sm.

Pitcairnia brevicalycina Mez

Pitcairnia brunnescens L.B. Sm.

Pitcairnia calatheoides L.B. Sm.

Pitcairnia cassapensis Mez
Argemone subfusiformis G.B. Ownbey

Bocconia integrifolia Bonpl.

\section{Passifloraceae}

Passiflora adenopoda DC.

Passiflora auriculata Kunth

Passiflora candollei Triana \& Planch.

Passiflora coccinea Aubl.

Passiflora cumbalensis (H. Karst.) Harms

Passiflora foetida $\mathrm{L}$.

Passiflora frutescens Ruiz \& Pav. ex Killip

Passiflora gracilens (A. Gray) Harms

Passiflora laurifolia $\mathrm{L}$.

Passiflora ligularis Juss.

Passiflora lobbii Mast.

Passiflora mixta L. f.

Passiflora parvifolia (DC.) Harms

Passiflora quadrangularis $\mathrm{L}$.

Passiflora quadriflora Killip

Passiflora rubra L.

Passiflora serratodigitata L.

Passiflora trifoliata Cav.

Passiflora triloba Ruiz \& Pav. ex DC.

Passiflora tripartita (Juss.) Poir.

Passiflora vespertilio $\mathrm{L}$.

Turnera orientalis (Urb.) Arbo

Turnera weddelliana Urb. \& Rolfe

\section{Pentaphylacaceae}

Freziera monsonensis (Melch.) Kobuski

Freziera spathulifolia (Melch.) Kobuski

Freziera tomentosa (Ruiz \& Pav.) Tul.

Ternstroemia globiflora Ruiz \& Pav.

Ternstroemia jelskii (SzyszyÅ..) Melch.

Ternstroemia pachytrocha Kobuski

Ternstroemia quinquepartita Ruiz \& Pav.

\section{Phrymaceae}

Erythranthe glabrata (Kunth) G.L. Nesom

Leucocarpus perfoliatus (Kunth) Benth.

\section{Phyllanthaceae}

Hieronyma alchorneoides Allemão

Hieronyma asperifolia Pax \& K. Hoffm.

Hieronyma duquei Cuatrec.

Hieronyma fendleri Briq.

Hieronyma macrocarpa Müll. Arg.

Hieronyma oblonga (Tul.) Müll. Arg.

Margaritaria nobilis L. $\mathrm{f}$.

Phyllanthus acuminatus Vahl

Phyllanthus brasiliensis (Aubl.) Poir.

Phyllanthus urinaria $\mathrm{L}$. 
Pitcairnia inermis (E. Mey.) E. Mey. ex Schult. \&

Schult. f.

Pitcairnia lanuginosa Ruiz \& Pav.

Pitcairnia paniculata (Ruiz \& Pav.) Ruiz \& Pav.

Pitcairnia pulverulenta Ruiz \& Pav.

Pitcairnia pungens Kunth

Pitcairnia straminea (Mez) Mez

Pitcairnia subulifera L.B. Sm.

Pitcairnia wolfei L.B. Sm.

Puya ferruginea (Ruiz \& Pav.) L.B. Sm.

Puya gracilis L.B. Sm.

Puya laccata $\mathrm{Mez}$

Puya lanuginosa (Ruiz \& Pav.) Schult. f.

Puya llatensis L.B. Sm.

Puya pyramidata (Ruiz \& Pav.) Schult. f.

Puya stipitata L.B. Sm.

Racinaea flexuosa (Baker) M.A. Spencer \& L.B. Sm.

Racinaea parviflora (Ruiz \& Pav.) M.A. Spencer \&

L.B. Sm.

Racinaea pendulispica (Mez) M.A. Spencer \& L.B. Sm.

Racinaea tetrantha (Ruiz \& Pav.) M.A. Spencer \&

L.B. Sm.

Ronnbergia explodens L.B. Sm.

Tillandsia biflora Ruiz \& Pav.

Tillandsia cacticola L.B. Sm.

Tillandsia capillaris Ruiz \& Pav.

Tillandsia clavigera Mez

Tillandsia complanata Benth.

Tillandsia didisticha (E. Morren) Baker

Tillandsia fendleri Griseb.

Tillandsia humilis C. Presl

Tillandsia ionochroma André ex Mez

Tillandsia juncea (Ruiz \& Pav.) Poir.

Tillandsia macbrideana L.B. Sm.

Tillandsia maculata Ruiz \& Pav.

Tillandsia oroyensis Mez

Tillandsia paraensis Mez

Tillandsia platyrhachis Mez

Tillandsia pyramidata André

Tillandsia recurvata (L.) L.

Tillandsia stenoura Harms

Tillandsia subconcolor L.B. Sm.

Tillandsia tectorum E. Morren

Tillandsia usneoides (L.) L.

Tillandsia wurdackii L.B. Sm.

Vriesea amazonica (Baker) Mez

Vriesea harmsiana (L.B. Sm.) L.B. Sm.

Vriesea olmosana L.B. Sm.

Vriesea rubra (Ruiz \& Pav.) Beer

Vriesea zamorensis (L.B. Sm.) L.B. Sm.

\section{Phytolaccaceae}

Hilleria latifolia (Lam.) H. Walter

Hilleria longifolia $(\mathrm{H}$. Walter) Heimerl

Hilleria secunda (Ruiz \& Pav.) H. Walter

Microtea debilis Sw.

Petiveria alliacea L.

Phytolacca bogotensis Kunth

Phytolacca rivinoides Kunth \& C.D. Bouché

Phytolacca weberbaueri $\mathrm{H}$. Walter

Schindleria densiflora (Kuntze) Monach.

Trichostigma octandrum (L.) H. Walter

Trichostigma peruvianum (Moq.) H. Walter

\section{Picramniaceae}

Nothotalisia peruviana (Standl.) W.W. Thomas

Picramnia killipii J.F. Macbr.

Picramnia latifolia Tul.

\section{Piperaceae}

Manekia incurva (Sieber ex Schult.) T. Arias, Callejas \& Bornst.

Peperomia acuminata Ruiz \& Pav.

Peperomia acutifolia C. DC.

Peperomia alata Ruiz \& Pav.

Peperomia areolata Trel.

Peperomia asplundii Yunck.

Peperomia blanda (Jacq.) Kunth

Peperomia bracteispica Trel.

Peperomia calvescens Trel.

Peperomia caniana Trel.

Peperomia casapiana C. DC.

Peperomia cerea Trel.

Peperomia ciliatifolia Trel.

Peperomia clavatispica Trel. \& Yunck.

Peperomia concava Ruiz \& Pav.

Peperomia confertispica Trel.

Peperomia curticaulis Trel.

Peperomia cushiana Trel.

Peperomia dependens Ruiz \& Pav.

Peperomia distachya (L.) A. Dietr.

Peperomia distachyos (L.) A. Dietr.

Peperomia drapeta Trel.

Peperomia ellipticorhombea C. DC.

Peperomia emarginulata C. DC.

Peperomia erythrocorma Trel.

Peperomia erythrostachya Trel.

Peperomia filiformis Ruiz \& Pav.

Peperomia fissicola Trel.

Peperomia foliiflora Ruiz \& Pav.

Peperomia furcata Opiz 


\section{Brunelliaceae}

Brunellia dulcis J.F. Macbr.

Brunellia inermis Ruiz \& Pav.

Brunellia weberbaueri Loes.

\section{Burmanniaceae}

Burmannia kalbreyeri Oliv.

Burmannia tenella Benth.

Cymbocarpa refracta Miers

Dictyostega orobanchoides (Hook.) Miers

Gymnosiphon breviflorus Gleason

Gymnosiphon divaricatus (Benth.) Benth. \& Hook. f.

Gymnosiphon suaveolens (H. Karst.) Urb.

Miersiella umbellata (Miers) Urb.

\section{Burseraceae}

Dacryodes belemensis Cuatrec.

Dacryodes peruviana (Loes.) H.J. Lam

Protium altsonii Sandwith

Protium apiculatum Swart

Protium aracouchini (Aubl.) Marchand

Protium calendulinum Daly

Protium crassipetalum Cuatrec.

Protium divaricatum Engl.

Protium glabrescens Swart

Protium opacum Swart

Protium puncticulatum J.F. Macbr.

Protium tenuifolium (Engl.) Engl.

Tetragastris panamensis (Engl.) Kuntze

Trattinnickia lawrancei Standl.

\section{Buxaceae}

Styloceras laurifolium (Willd.) Kunth

\section{Cactaceae}

Corryocactus chavinilloensis F. Ritter

Corryocactus quivillanus F. Ritter

Cylindropuntia tunicata (Lehm.) F.M. Knuth

Echinopsis tulhuayacensis (Ochoa ex Backeb.)

Friedrich \& G.D. Rowley

Espostoa lanata (Kunth) Britton \& Rose

Opuntia quitensis F.A.C. Weber

Rhipsalis baccifera (Sol.) Stearn

\section{Calceolariaceae}

Calceolaria bicolor Ruiz \& Pav.

Calceolaria bicrenata Ruiz \& Pav.

Calceolaria cajabambae Kraenzl.

Calceolaria calycina Benth.

Calceolaria chelidonioides Kunth

Calceolaria conocarpa Pennell

Calceolaria cuneiformis Ruiz \& Pav.

Calceolaria deflexa Ruiz \& Pav.

Calceolaria flexuosa Ruiz \& Pav.

Calceolaria heterophylla Ruiz \& Pav.
Peperomia fuscispica C. DC.

Peperomia galioides Kunth

Peperomia glabella (Sw.) A. Dietr.

Peperomia heterophylla Miq.

Peperomia heterostachya A. Dietr.

Peperomia hispidula (Sw.) A. Dietr.

Peperomia hispiduliformis Trel.

Peperomia hoffmannii C. DC.

Peperomia huallagana Trel.

Peperomia huanucoana Trel.

Peperomia inaequalifolia Ruiz \& Pav.

Peperomia inaequilatera Trel.

Peperomia lanceolatopeltata C. DC.

Peperomia lancifolia Hook.

Peperomia lanosa Trel.

Peperomia leuconeura Trel.

Peperomia longipedunculata Opiz

Peperomia macbrideana Trel.

Peperomia macrostachyos (Vahl) A. Dietr.

Peperomia maculosa (L.) Hook.

Peperomia marshalliana Trel.

Peperomia martiana Miq.

Peperomia mathewsiana Miq.

Peperomia megalepis Trel.

Peperomia micromamillata Trel.

Peperomia monostachya Ruiz \& Pav.

Peperomia nigrooculata Trel.

Peperomia nivalis Miq.

Peperomia obex Trel.

Peperomia obliqua Ruiz \& Pav.

Peperomia obtusifolia (L.) A. Dietr.

Peperomia pachiteana Trel.

Peperomia parvifolia C. DC.

Peperomia pearcei Trel.

Peperomia pellucida (L.) Kunth

Peperomia peltoidea Kunth

Peperomia peruviana Dahlst.

Peperomia pilifera Trel.

Peperomia pilosa Ruiz \& Pav.

Peperomia playapampana Trel.

Peperomia plurispica Trel.

Peperomia procumbens C. DC.

Peperomia pseudorhynchophoros C. DC.

Peperomia pseudosalicifolia Trel.

Peperomia puberulipes Trel.

Peperomia pumila Opiz

Peperomia purpurea Ruiz \& Pav.

Peperomia quaesita Trel.

Peperomia retusa (L. f.) A. Dietr. 
Calceolaria incarum Kraenzl. Calceolaria inflexa Ruiz \& Pav. Calceolaria linearis Ruiz \& Pav. Calceolaria maculata Edwin Calceolaria mexicana Benth. Calceolaria olivacea Molau Calceolaria pavonii Benth. Calceolaria phaceliifolia Edwin Calceolaria punicea Ruiz \& Pav. Calceolaria salicifolia Ruiz \& Pav. Calceolaria scapiflora (Ruiz \& Pav.) Benth. Calceolaria tenuis Benth. Calceolaria tomentosa Ruiz \& Pav. Calceolaria tripartita Ruiz \& Pav. Calceolaria virgata Ruiz \& Pav. Calceolaria viscosa Ruiz \& Pav.

\section{Calophyllaceae}

Marila laxiflora Rusby

\section{Campanulaceae}

Centropogon astrotrichus E. Wimm.

Centropogon caoutchouc (Kunth) Gleason

Centropogon capitatus Drake

Centropogon cornutus (L.) Druce

Centropogon erianthus (Benth.) Benth. \& Hook. f.

Centropogon featherstonei Gleason

Centropogon fuscus (G. Don) E. Wimm.

Centropogon gleasonii (E. Wimm.) E. Wimm.

Centropogon granulosus C. Presl

Centropogon hirtus (Cav.) C. Presl

Centropogon hypotrichus E. Wimm.

Centropogon latifolius E. Wimm.

Centropogon luteus E. Wimm.

Centropogon macbridei Gleason

Centropogon macrocarpus Zahlbr.

Centropogon macrophyllus (G. Don) E. Wimm.

Centropogon reflexus C. Presl

Centropogon sciaphilus Zahlbr.

Centropogon umbrosus E. Wimm.

Centropogon verbascifolius (C. Presl) Gleason

Centropogon weberbaueri Zahlbr.

Centropogon yungasensis Britton

Diastatea micrantha (Kunth) McVaugh

Hippobroma longiflora (L.) G. Don

Lobelia holotricha E. Wimm.

Lobelia oligophylla (Wedd.) Lammers

Lobelia xalapensis Kunth

Lysipomia brachysiphonia E. Wimm.

Lysipomia globularis E. Wimm.

Lysipomia multiflora McVaugh

Siphocampylus angustiflorus Schltdl. ex Zahlbr.
Peperomia rhombea Ruiz \& Pav.

Peperomia rhombeoelliptica Trel.

Peperomia rhombilimba Trel.

Peperomia rosea Trel.

Peperomia rotundifolia (L.) Kunth

Peperomia rubrispica Trel.

Peperomia scabiosa Trel.

Peperomia secunda Ruiz \& Pav.

Peperomia septemnervis Ruiz \& Pav.

Peperomia serpens (Sw.) Loudon

Peperomia striata Ruiz \& Pav.

Peperomia sylvatica C. DC.

Peperomia tenuiramea C. DC.

Peperomia tetragona Ruiz \& Pav.

Peperomia trichopus Trel.

Peperomia tricolor Trel.

Peperomia trinervis Ruiz \& Pav.

Peperomia variculata Trel.

Peperomia yananoensis Trel.

Piper acutifolium Ruiz \& Pav.

Piper admirabile Yunck.

Piper aduncum L.

Piper aequale Vahl

Piper albiciliatum Yunck.

Piper alveolatum Opiz

Piper apodum Trel.

Piper arboreum Aubl.

Piper asplundii Yunck.

Piper asterotrichum C. DC.

Piper augustum Rudge

Piper brasiliense C. DC.

Piper brevispicum C. DC.

Piper brongniartii (Miq.) C. DC.

Piper callosum Ruiz \& Pav.

Piper camphoriferum C. DC.

Piper carpunya Ruiz \& Pav.

Piper casapiense (Miq.) C. DC.

Piper cassinoides Opiz

Piper cernuum Vell.

Piper churumayu Ruiz \& Pav.

Piper conispicum Trel.

Piper controversum Steud.

Piper coruscans Kunth

Piper costatum C. DC.

Piper crassinervium Kunth

Piper crocatum Ruiz \& Pav.

Piper curvatum Ruiz \& Pav.

Piper dasyoura (Miq.) C. DC.

Piper demeraranum (Miq.) C. DC.

Piper dichotomum Ruiz \& Pav. 
Siphocampylus arachnes E. Wimm. Siphocampylus chloroleucus E. Wimm. Siphocampylus comosus G. Don Siphocampylus dependens G. Don Siphocampylus fissus Gleason Siphocampylus floribundus Zahlbr. Siphocampylus lobbii Zahlbr. Siphocampylus ovatus (G. Don) E. Wimm. Siphocampylus parvilobus E. Wimm. Siphocampylus pozuzensis E. Wimm. Siphocampylus tupaeformis Zahlbr. Wahlenbergia linarioides (Lam.) A. DC.

\section{Cannabaceae}

Celtis iguanaea (Jacq.) Sarg.

Celtis schippii Standl.

Lozanella enantiophylla (Donn. Sm.) Killip \& C.V.

Morton

Lozanella permollis Killip \& C.V. Morton

Trema micrantha (L.) Blume

\section{Cannaceae}

Canna indica L.

Canna iridiflora Ruiz \& Pav.

Canna jaegeriana Urb.

Canna paniculata Ruiz \& Pav.

\section{Capparaceae}

Capparidastrum osmanthum (Diels) Cornejo \& Iltis Capparidastrum sola (J.F. Macbr.) Cornejo \& H. Iltis Capparis detonsa Triana \& Planch.

\section{Caprifoliaceae}

Astrephia chaerophylloides (Sm.) DC.

Belonanthus angustifolius Schmale

Phyllactis rigida (Ruiz \& Pav.) Pers.

Phyllactis tenuifolia (Ruiz \& Pav.) Pers.

Valeriana baltana Graebn.

Valeriana bambusicaulis Killip

Valeriana clematitis Kunth

Valeriana coarctata Ruiz \& Pav.

Valeriana condamoana Graebn.

Valeriana decussata Ruiz \& Pav.

Valeriana globiflora Ruiz \& Pav.

Valeriana globularis A. Gray

Valeriana henrici (Graebn.) B. Eriksen

Valeriana isoetifolia Killip

Valeriana ledoides Graebn.

Valeriana macbridei Killip

Valeriana oblongifolia Ruiz \& Pav.

Valeriana paniculata Ruiz \& Pav.

Valeriana rufescens Killip

Valeriana scandens $\mathrm{L}$.

Valeriana spathulata Ruiz \& Pav.
Piper divaricatum G. Mey.

Piper diversipilum Trel.

Piper dulce Trel.

Piper durvilleanum Trel.

Piper elongatum Vahl

Piper endlicherianum Trel.

Piper excavatum Ruiz \& Pav.

Piper factum Trel.

Piper ferreyrae Yunck.

Piper formosum (Miq.) C. DC.

Piper fulgentifolium Yunck.

Piper gracile Ruiz \& Pav.

Piper granulosum Ruiz \& Pav.

Piper griseum C. DC.

Piper heterophyllum Ruiz \& Pav.

Piper hispidum Sw.

Piper huacachianum Trel.

Piper huallaganum Trel.

Piper huantanum Trel.

Piper immutatum Trel.

Piper indecorum Kunth

Piper kanehiranum Trel.

Piper laevigatum Kunth

Piper lanceifolium Kunth

Piper lanceolatum Ruiz \& Pav.

Piper lehmannianum (Miq.) C. DC.

Piper leticianum C. DC.

Piper lineatum Ruiz \& Pav.

Piper lineolatifolium Trel. \& Yunck.

Piper Ilatanum Trel.

Piper longifolium Ruiz \& Pav.

Piper magnificum Trel.

Piper malifolium Trel.

Piper mellibracteaum Trel.

Piper mite Ruiz \& Pav.

Piper monzonense C. DC.

Piper multiplinervium C. DC.

Piper munyanum Trel.

Piper neglectum Trel.

Piper nudilimbum C. DC.

Piper obesum Vahl

Piper obliquum Ruiz \& Pav.

Piper oblongatum Opiz

Piper obtusilimbum C. DC.

Piper opizii (Kunth) Steud.

Piper papillicaule Trel. \& Yunck.

Piper patens Opiz

Piper peltatum L.

Piper perareolatum C. DC. 
Valeriana tessendorffiana Graebn.

Valeriana urticifolia Kunth

Valeriana verrucosa Schmale

Valeriana warburgii Graebn.

Valeriana weberbaueri Graebn.

\section{Cardiopteridaceae}

Citronella incarum (J.F. Macbr.) R.A. Howard

Dendrobangia multinervia Ducke

\section{Caricaceae}

Carica papaya L.

Jacaratia digitata (Poepp. \& Endl.) Solms

Jacaratia spinosa (Aubl.) A. DC.

Vasconcellea glandulosa A. DC.

Vasconcellea microcarpa (Jacq.) A. DC.

Vasconcellea monoica (Desf.) A. DC.

Vasconcellea parviflora A. DC.

Vasconcellea pubescens A. DC.

\section{Caryocaraceae}

Anthodiscus peruanus Baill.

Caryocar amygdaliforme Ruiz \& Pav. ex G. Don

Caryocar glabrum (Aubl.) Pers.

\section{Caryophyllaceae}

Arenaria bisulca (Bartl.) Fenzl \& Rohrb.

Arenaria lanuginosa (Michx.) Rohrb.

Arenaria poeppigiana Rohrb.

Cardionema ramosissimum (Weinm.) A. Nelson \&

J.F. Macbr.

Cerastium crassipes Bartl.

Cerastium danguyi J.F. Macbr.

Cerastium mollissimum Poir.

Cerastium subspicatum Wedd.

Dianthus caryophyllus L.

Drymaria auriculipetala Mattf.

Drymaria cordata (L.) Willd. ex Schult.

Drymaria glaberrima Bartl.

Drymaria grandiflora Bartl.

Drymaria ovata Humb. \& Bonpl. ex Schult.

Drymaria stereophylla Mattf.

Drymaria villosa Schltdl. \& Cham.

Paronychia andina A. Gray

Paronychia macbridei Chaudhri

Silene gallica L.

Silene genovevae Bocquet

Stellaria cuspidata Willd. ex D.F.K. Schltdl.

Stellaria media (L.) Vill.

Stellaria nemorum L.

Stellaria recurvata Willd. ex D.F.K. Schltdl.

\section{Celastraceae}

Anthodon decussatum Ruiz \& Pav.

Cheiloclinium cognatum (Miers) A.C. Sm.
Piper peruvianum (Miq.) C. DC.

Piper poporense Trel. \& Yunck.

Piper posusanum Trel.

Piper pseudocallosum Trel.

Piper pseudomatico Trel.

Piper pseudomollicomum C. DC.

Piper puberulescens Trel.

Piper puberuliciliatum Yunck.

Piper pubibaccum C. DC.

Piper restio Trel.

Piper reticulatum $\mathrm{L}$.

Piper riojanum Trel.

Piper roqueanum Trel.

Piper rubribaccum Trel.

Piper rusbyi C. DC.

Piper schiedeanum Steud.

Piper sciaphilum C. DC.

Piper secundum Ruiz \& Pav.

Piper sellertianum Trel.

Piper serrulatum Yunck.

Piper sinuclausum Trel.

Piper sororium (Miq.) C. DC.

Piper subandinum C. DC.

Piper subpatens Trel.

Piper subsilvestre C. DC.

Piper tenuicuspe (Miq.) C. DC.

Piper tocacheanum C. DC.

Piper trichostylopse Trel.

Piper tuberculatum Jacq.

Piper udisilvestre C. DC.

Piper umbellatum $\mathrm{L}$.

Piper unguiculatum Ruiz \& Pav.

Piper verbascifolium (Miq.) C. DC.

Piper volubile C. DC.

Piper yananoanum Trel.

\section{Plantaginaceae}

Basistemon intermedius Edwin

Conobea scoparioides (Cham. \& Schltdl.) Benth.

Ourisia muscosa Wedd.

Plantago australis Lam.

Plantago lamprophylla Pilg.

Plantago limensis Pers.

Plantago major L.

Plantago myosuros Lam.

Plantago orbignyana Steinh. ex Decne.

Plantago rigida Kunth

Plantago sericea Ruiz \& Pav.

Plantago tubulosa Decne.

Scoparia dulcis L. 
Cheiloclinium hippocrateoides (Peyr.) A.C. Sm. Haydenoxylon urbanianum (Loes.) M.P. Simmons Hippocratea volubilis $\mathrm{L}$.

Maytenus boarioides Loes.

Maytenus conferta (Ruiz \& Pav.) Reissek ex Loes.

Maytenus macrocarpa (Ruiz \& Pav.) Briq.

Maytenus pseudoboaria Loes.

Maytenus verticillata (Ruiz \& Pav.) DC.

Peritassa huanucana (Loes.) A.C. Sm.

Peritassa laevigata (Hoffmanns. ex Link) A.C. Sm.

Salacia juruana Loes.

Tontelea coriacea A.C. Sm.

Tontelea passiflora (Vell.) Lombardi

\section{Chloranthaceae}

Hedyosmum angustifolium (Ruiz \& Pav.) Solms

Hedyosmum cuatrecazanum Occhioni

Hedyosmum dombeyanum Solms

Hedyosmum lechleri Solms

Hedyosmum peruvianum Todzia

Hedyosmum racemosum (Ruiz \& Pav.) G. Don

Hedyosmum scabrum (Ruiz \& Pav.) Solms

Hedyosmum spectabile Todzia

Hedyosmum sprucei Solms

\section{Chrysobalanaceae}

Couepia chrysocalyx (Poepp.) Benth. ex Hook. f.

Hirtella excelsa Standl. ex Prance

Hirtella hispidula Miq.

Hirtella racemosa Lam.

Hirtella triandra Sw.

Licania arborea Seem.

Licania bullata Prance

Licania durifolia Cuatrec.

Licania hirsuta Prance

Licania macrocarpa Cuatrec.

\section{Cleomaceae}

Andinocleome lechleri (Eichler) Iltis \& Cochrane

Cleome glandulosa Ruiz \& Pav. ex DC.

Cleome limoneolens J.F. Macbr.

Cleome longifolia C. Presl

Cleome pachystigma Briq.

Hemiscola aculeata (L.) Raf.

Melidiscus giganteus (L.) Raf.

Podandrogyne hispidula (DC.) Cochrane

Podandrogyne mathewsii (Briq.) Cochrane

Tarenaya longipes (DC.) Iltis

\section{Clethraceae}

Clethra ferruginea (Ruiz \& Pav.) Link ex Spreng.

Clethra obovata (Ruiz \& Pav.) G. Don

Clethra pedicellaris Turcz.
Sibthorpia repens (L.) Kuntze

Veronica serpyllifolia L.

\section{Plumbaginaceae}

Plumbago coerulea Kunth

Plumbago zeylanica L.

\section{Poaceae}

Aciachne pulvinata Benth.

Acroceras zizanioides (Kunth) Dandy

Agrostis boliviana Mez

Agrostis breviculmis Hitchc.

Agrostis gigantea Roth

Agrostis mertensii Trin.

Agrostis perennans (Walter) Tuck.

Agrostis tolucensis Kunth

Andropogon bicornis L.

Andropogon lateralis Nees

Aristida adscensionis L.

Aristida torta (Nees) Kunth

Arundinella berteroniana (Schult.) Hitchc. \& Chase

Arundinella hispida (Humb. \& Bonpl. ex Willd.) Kuntze

Aulonemia haenkei (Rupr.) McClure

Aulonemia hirtula (Pilg.) McClure

Aulonemia parviflora (J. Presl) McClure

Aulonemia patula (Pilg.) McClure

Aulonemia queko Goudot

Avena sativa L.

Avena sterilis $\mathrm{L}$.

Axonopus capillaris (Lam.) Chase

Axonopus compressus (Sw.) P. Beauv.

Axonopus elegantulus (J. Presl) Hitchc.

Axonopus scoparius (Flüggé) Kuhlm.

Bothriochloa barbinodis (Lag.) Herter

Bothriochloa saccharoides (Sw.) Rydb.

Bouteloua curtipendula (Michx.) Torr.

Briza minor L.

Bromus catharticus Vahl

Bromus lanatus Kunth

Bromus villosissimus Hitchc.

Calamagrostis alba (J. Presl) Steud.

Calamagrostis fibrovaginata Lægaard

Calamagrostis jamesonii Steud.

Calamagrostis ligulata (Kunth) Hitchc.

Calamagrostis macrophylla (Pilg.) Pilg.

Cenchrus brownii Roem. \& Schult.

Cenchrus myosuroides Kunth

Cenchrus peruvianus (Trin.) Morrone

Chascolytrum monandrum (Hack.) L. Essi, Longhi-

Wagner \& Souza-Chies

Chloris pycnothrix Trin.

Chloris virgata Sw. 
Clethra revoluta (Ruiz \& Pav.) Spreng.

\section{Clusiaceae}

Chrysochlamys ulei Engl.

Chrysochlamys weberbaueri Engl.

Clusia cassinioides Planch. \& Triana

Clusia flavida (Benth.) Pipoly

Clusia minor L.

Clusia pseudochina Poepp. \& Endl.

Garcinia macrophylla Mart.

Rheedia brasiliensis (Mart.) Planch. \& Triana

Symphonia globulifera L. f.

Tovomita microcarpa Walp.

Tovomita weddelliana Planch. \& Triana

\section{Columelliaceae}

Columellia oblonga Ruiz \& Pav.

\section{Combretaceae}

Combretum fruticosum (Loefl.) Stuntz

Terminalia amazonia (J.F. Gmel.) Exell

Terminalia oblonga (Ruiz \& Pav.) Steud.

\section{Commelinaceae}

Callisia gracilis (Kunth) D.R. Hunt

Callisia repens (Jacq.) L.

Commelina erecta $\mathrm{L}$.

Commelina fasciculata Ruiz \& Pav.

Commelina hispida Ruiz \& Pav.

Commelina rufipes Seub.

Commelina tuberosa L.

Dichorisandra hexandra (Aubl.) Kuntze ex Hand.-

Mazz.

Dichorisandra ulei J.F. Macbr.

Floscopa elegans Huber

Floscopa peruviana Hassk. ex C.B. Clarke

Floscopa robusta (Seub.) C.B. Clarke

Gibasis geniculata (Jacq.) Rohweder

Tinantia erecta (Jacq.) Schltdl.

Tradescantia cymbispatha C.B. Clarke

Tradescantia zebrina Heynh. ex Bosse

Tripogandra multiflora (Sw.) Raf.

Tripogandra serrulata (Vahl) Handlos

\section{Connaraceae}

Connarus bracteosovillosus Forero

Connarus punctatus Planch.

Rourea amazonica (Baker) Radlk.

\section{Convolvulaceae}

Calycobolus sericeus (Kunth) House

Convolvulus crenatifolius Ruiz \& Pav.

Cuscuta corymbosa Ruiz \& Pav.

Cuscuta foetida Kunth

Cuscuta grandiflora Kunth

Cuscuta odorata Ruiz \& Pav.
Chondrosum simplex (Lag.) Kunth

Chusquea aspera L.G. Clark

Chusquea depauperata Pilg.

Chusquea picta Pilg.

Chusquea scandens Kunth

Chusquea tessellata Munro

Cinna poiformis (Kunth) Scribn. \& Merr.

Coix lacryma-jobi L.

Cortaderia bifida Pilg.

Cortaderia jubata (Lemoine) Stapf

Cortaderia planifolia Swallen

Cortaderia sericantha (Steud.) Hitchc.

Cottea pappophoroides Kunth

Cryptochloa unispiculata Soderstr.

Dactylis glomerata L.

Deyeuxia brevifolia J. Presl

Deyeuxia chrysantha J. Presl

Deyeuxia curvula Wedd.

Deyeuxia densiflora J. Presl

Deyeuxia eminens J. Presl

Deyeuxia fuscata J. Presl

Deyeuxia heterophylla Wedd.

Deyeuxia ovata J. Presl

Deyeuxia pooides (Steud.) Rúgolo

Deyeuxia recta Kunth

Deyeuxia rigescens (J. Presl) Türpe

Deyeuxia rigida Kunth

Deyeuxia rupestris (Trin.) Rúgolo

Deyeuxia spicigera J. Presl

Deyeuxia tarmensis (Pilg.) Sodiro

Deyeuxia vicunarum Wedd.

Digitaria ciliaris (Retz.) Koeler

Echinochloa polystachya (Kunth) Hitchc.

Eragrostis japonica (Thunb.) Trin.

Eragrostis magna Hitchc.

Eragrostis nigricans (Kunth) Steud.

Eragrostis pastoensis (Kunth) Trin.

Eragrostis patula (Kunth) Steud.

Festuca carazana Pilg.

Festuca casapaltensis Ball

Festuca compressifolia J. Presl

Festuca dentiflora E.B. Alexeev ex StanÄ• ík \& P.M.

Peterson

Festuca dichoclada Pilg.

Festuca distichovaginata Pilg.

Festuca dolichophylla J. Presl

Festuca hieronymi Hack.

Festuca huamachucensis Infantes

Festuca inarticulata Pilg.

Festuca longigluma Tovar 
Dichondra microcalyx (Hallier f.) Fabris

Evolvulus argyreus Choisy

Evolvulus herrerae Ooststr.

Evolvulus incanus Pers.

Ipomoea alba L.

Ipomoea batatas (L.) Lam.

Ipomoea clavata (G. Don) Ooststr. ex J.F. Macbr.

Ipomoea dubia Roem. \& Schult.

Ipomoea lindenii M. Martens \& Galeotti

Ipomoea muricata (L.) Jacq.

Ipomoea nil (L.) Roth

Ipomoea philomega (Vell.) House

Ipomoea purpurea (L.) Roth

Ipomoea ramosissima (Poir.) Choisy

Ipomoea squamosa Choisy

Ipomoea tricolor Cav.

Iseia luxurians (Moric.) O'Donell

Jacquemontia blanchetii Moric.

Jacquemontia ciliata Sandwith

Jacquemontia elegans Helwig

Merremia macrocalyx (Ruiz \& Pav.) O'Donell

Merremia quinquefolia (L.) Hallier $\mathrm{f}$.

Merremia umbellata (L.) Hallier $\mathrm{f}$.

Odonellia hirtiflora (M. Martens \& Galeotti) K.R.

Robertson

Turbina corymbosa (L.) Raf.

\section{Coriariaceae}

Coriaria ruscifolia L.

\section{Cornaceae}

Cornus peruviana J.F. Macbr.

\section{Costaceae}

Costus guanaiensis Rusby

Costus laevis Ruiz \& Pav.

Costus lasius Loes.

Costus scaber Ruiz \& Pav.

Costus spiralis (Jacq.) Roscoe

Dimerocostus argenteus (Ruiz \& Pav.) Maas

Dimerocostus strobilaceus Kuntze

\section{Crassulaceae}

Crassula venezuelensis (Steyerm.) M. Bywater \&

Wickens

Echeveria chiclensis (Ball) Berger

Sedum reniforme (H. Jacobsen) Thiede \& 't Hart

Villadia reniformis $\mathrm{H}$. Jacobsen

\section{Cucurbitaceae}

Apodanthera mandonii Cogn.

Apodanthera mathewsii Arn.

Apodanthera mucronata Cogn.
Festuca loricata (Griseb.) Pilg.

Festuca peruviana Infantes

Festuca procera Kunth

Festuca pubigluma Tovar

Festuca rigescens (J. Presl) Kunth

Festuca tarmensis Pilg.

Festuca weberbaueri Pilg.

Gouinia virgata (J. Presl) Scribn.

Gynerium sagittatum (Aubl.) P. Beauv.

Hemarthria altissima (Poir.) Stapf \& C.E. Hubb.

Heteropogon contortus (L.) P. Beauv. ex Roem. \&

Schult.

Heteropogon melanocarpus (Elliott) Benth.

Homolepis glutinosa (Sw.) Zuloaga \& Soderstr.

Hordeum vulgare L.

Hymenachne amplexicaulis (Rudge) Nees

Ichnanthus nemorosus (Sw.) Döll

Ichnanthus pallens (Sw.) Munro ex Benth.

Imperata brasiliensis Trin.

Isachne arundinacea (Sw.) Griseb.

Ischaemum rugosum Salisb.

Jarava ichu Ruiz \& Pav.

Jarava macbridei (Hitchc.) Peñail.

Lamprothyrsus hieronymi (Kuntze) Pilg.

Lasiacis ligulata Hitchc. \& Chase

Lasiacis maculata (Aubl.) Urb.

Lasiacis oaxacensis (Steud.) Hitchc.

Lasiacis procerrima (Hack.) Hitchc.

Lasiacis scabrior Hitchc.

Leptochloa fusca (L.) Kunth

Leptochloa virgata (L.) P. Beauv.

Lolium multiflorum Lam.

Megathyrsus maximus (Jacq.) B.K. Simon \& S.W.L.

Jacobs

Melica scabra Kunth

Melinis minutiflora P. Beauv.

Muhlenbergia angustata (J. Presl) Kunth

Muhlenbergia cenchroides (Humb. \& Bonpl. ex Willd.)

P.M. Peterson

Muhlenbergia ciliata (Kunth) Trin.

Muhlenbergia flexuosa Hitchc.

Muhlenbergia ligularis (Hack.) Hitchc.

Muhlenbergia microsperma (DC.) Kunth

Muhlenbergia peruviana (P. Beauv.) Steud.

Muhlenbergia rigida (Kunth) Kunth

Muhlenbergia tenuifolia (Kunth) Kunth

Nassella ayacuchensis (Tovar) Barkworth

Nassella brachyphylla (Hitchc.) Barkworth

Nassella huallancaensis (Tovar) Barkworth 
Apodanthera tumbeziana Harms

Calycophysum pedunculatum $\mathrm{H}$. Karst. \& Triana

Cayaponia glandulosa (Poepp. \& Endl.) Cogn.

Cayaponia ophthalmica R.E. Schult.

Cayaponia peruviana (Poepp. \& Endl.) Cogn.

Cayaponia ruizii Cogn.

Cucurbita maxima Duchesne

Cyclanthera cordifolia Cogn.

Cyclanthera pedata (L.) Schrad.

Cyclanthera tamnifolia Griseb.

Fevillea cordifolia L.

Gurania bignoniacea (Poepp. \& Endl.) C. Jeffrey

Gurania capitata (Poepp. \& Endl.) Cogn.

Gurania eriantha (Poepp. \& Endl.) Cogn.

Gurania lobata (L.) Pruski

Gurania rhizantha (Poepp. \& Endl.) C. Jeffrey

Gurania sinuata (Benth.) Cogn.

Melothria warmingii Cogn.

Psiguria triphylla (Miq.) C. Jeffrey

Rytidostylis trianaei (Cogn.) Kuntze

Sechium edule (Jacq.) Sw.

Sicyos gracillimus Cogn.

Sicyos malvifolius Griseb.

Sicyos montanus Poepp. \& Endl.

Sicyos urolobus Harms

\section{Culcitaceae}

Culcita coniifolia (Hook.) Maxon

\section{Cunoniaceae}

Weinmannia auriculata D. Don

Weinmannia cinerea Ruiz \& Pav.

Weinmannia cochensis Hieron.

Weinmannia crassifolia Ruiz \& Pav.

Weinmannia elliptica Kunth

Weinmannia fagaroides Kunth

Weinmannia glomerata C. Presl

Weinmannia haenkeana Engl.

Weinmannia heterophylla Kunth

Weinmannia latifolia C. Presl

Weinmannia lechleriana Engl.

Weinmannia lentiscifolia C. PresI

Weinmannia ovata Cav.

Weinmannia pentaphylla Ruiz \& Pav.

Weinmannia pubescens Kunth

Weinmannia reticulata Ruiz \& Pav.

Weinmannia subsessiliflora Ruiz \& Pav.

Weinmannia trichosperma Cav.

\section{Cyatheaceae}

Alsophila cuspidata (Kunze) D.S. Conant
Nassella inconspicua (J. Presl) Barkworth

Nassella mexicana (Hitchc.) R.W. Pohl

Nassella mucronata (Kunth) R.W. Pohl

Olyra caudata Trin.

Olyra fasciculata Trin.

Olyra latifolia L.

Oplismenus hirtellus (L.) P. Beauv.

Oryza sativa L.

Panicum grande Hitchc. \& Chase

Panicum hirticaule J. Presl

Panicum parvifolium Lam.

Panicum stoloniferum Poir.

Panicum stramineum Hitchc. \& Chase

Panicum trichoides Sw.

Pappophorum pappiferum (Lam.) Kuntze

Pariana bicolor Tutin

Pariana campestris Aubl.

Pariana stenolemma Tutin

Parodiolyra lateralis (J. Presl ex Nees) Soderstr. \&

Zuloaga

Paspalum candidum (Humb. \& Bonpl. ex Flüggé)

Kunth

Paspalum ceresia (Kuntze) Chase

Paspalum conjugatum P.J. Bergius

Paspalum conspersum Schrad.

Paspalum decumbens Sw.

Paspalum distichum $\mathrm{L}$.

Paspalum haenkeanum J. Presl

Paspalum heterotrichon Trin.

Paspalum notatum Flüggé

Paspalum paniculatum $\mathrm{L}$.

Paspalum penicillatum Hook. $\mathrm{f}$.

Paspalum pilgerianum Chase

Paspalum plenum Chase

Paspalum plicatulum Michx.

Paspalum racemosum Lam.

Paspalum saccharoides Nees ex Trin.

Paspalum virgatum $\mathrm{L}$.

Pennisetum bambusiforme (E. Fourn.) Hemsl. ex B.D. Jacks.

Pharus lappulaceus Aubl.

Pharus latifolius $\mathrm{L}$.

Pharus virescens Döll

Piresia sympodica (Döll) Swallen

Poa aequatoriensis Hack.

Poa anae Tovar

Poa annua L.

Poa calycina (J. Presl) Kunth

Poa deminuta Refulio

Poa fibrifera Pilg.

Poa glaberrima Tovar 
Cyathea alatissima (Stolze) Lehnert

Cyathea andina (H. Karst.) Domin

Cyathea bipinnatifida (Baker) Domin

Cyathea caracasana (Klotzsch) Domin

Cyathea conjugata (Spruce ex Hook.) Domin

Cyathea delgadii Sternb.

Cyathea divergens Kunze

Cyathea frigida (H. Karst.) Domin

Cyathea lasiosora (Mett. ex Kuhn) Domin

Cyathea lechleri Mett.

Cyathea nigripes (C. Chr.) Domin

Cyathea petiolata (Hook.) R.M. Tryon

Cyathea pilosissima (Baker) Domin

Cyathea poeppigii (Hook.) Domin

Cyathea pungens (Willd.) Domin

Cyathea ruiziana Klotzsch

Cyathea subtropica Domin

Sphaeropteris quindiuensis (H. Karst.) R.M. Tryon

\section{Cyclanthaceae}

Asplundia acuminata (Ruiz \& Pav.) Harling

Asplundia ecuadoriensis (Harling) Harling

Asplundia flavovaginata Harling

Asplundia latifolia (Ruiz \& Pav.) Harling

Asplundia parviflora Harling

Asplundia platanthera Harling

Asplundia schizotepala Harling

Carludovica palmata Ruiz \& Pav.

Cyclanthus bipartitus Poit. ex A. Rich.

Dicranopygium latissimum Harling

Dicranopygium yacu-sisa Harling

Evodianthus funifer (Poit.) Lindm.

Ludovia lancifolia Brongn.

Sphaeradenia angustifolia (Ruiz \& Pav.) Harling

Sphaeradenia crassiceps R. Erikss.

Sphaeradenia rhodocephala (Harling) Harling

Thoracocarpus bissectus (Vell.) Harling

\section{Cyperaceae}

Bulbostylis capillaris (L.) Kunth ex C.B. Clarke

Bulbostylis tenuifolia (Rudge) J.F. Macbr.

Calyptrocarya bicolor (H. Pfeiff.) T. Koyama

Calyptrocarya glomerulata (Brongn.) Urb.

Carex acutata Boott

Carex boliviensis Van Heurck \& Müll. Arg.

Carex bonplandii Kunth

Carex brachycalama Griseb.

Carex crinalis Boott

Carex fecunda Steud.

Carex hebetata Boott

Carex humahuacaensis G.A. Wheeler
Poa horridula Pilg.

Poa humillima Pilg.

Poa lilloi Hack.

Poa linearifolia Refulio

Poa scabrivaginata Tovar

Polypogon elongatus Kunth

Polypogon exasperatus (Trin.) Renvoize

Polypogon interruptus Kunth

Polypogon magellanicus (Lam.) Finot

Pseudechinolaena polystachya (Kunth) Stapf

Rottboellia cochinchinensis (Lour.) Clayton

Rugoloa polygonata (Schrad.) Zuloaga

Schizachyrium condensatum (Kunth) Nees

Schizachyrium sanguineum (Retz.) Alston

Schizachyrium tenerum Nees

Setaria vulpiseta (Lam.) Roem. \& Schult.

Sporobolus indicus (L.) R. Br.

Sporobolus lasiophyllus Pilg.

Sporobolus pyramidalis P. Beauv.

Steinchisma laxum (Sw.) Zuloaga

Trachypogon spicatus (L. f.) Kuntze

Tragus berteronianus Schult.

Triniochloa stipoides (Kunth) Hitchc.

Tripsacum andersonii J.R. Gray

Tripsacum australe H.C. Cutler \& E.S. Anderson

Triticum aestivum $\mathrm{L}$.

Urochloa fusca (Sw.) B.F. Hansen \& Wunderlin

Urochloa mutica (Forssk.) T.Q. Nguyen

Vulpia myuros (L.) C.C. Gmel.

Zea mays L.

\section{Podocarpaceae}

Podocarpus glomeratus D. Don

Podocarpus magnifolius J. Buchholz \& N.E. Gray

Podocarpus oleifolius D. Don ex Lamb.

\section{Polemoniaceae}

Cantua buxifolia Juss. ex Lam.

Cantua flexuosa (Ruiz \& Pav.) Pers.

\section{Polygalaceae}

Asemeia acuminata (Willd.) J.F.B. Pastore \& J.R.

Abbott

Caamembeca gigantea (Chodat) J.F.B. Pastore

Monnina amplibracteata Ferreyra

Monnina conferta Ruiz \& Pav.

Monnina macrostachya Ruiz \& Pav.

Monnina marginata C. Presl

Monnina ovata Ferreyra

Monnina pavoni Chodat

Monnina polygonoides Chodat

Monnina polystachya Ruiz \& Pav.

Monnina pseudopolystachya Chodat 
Carex jamesonii Boott Carex microglochin Wahlenb.

Carex peucophila Holm

Carex pichinchensis Kunth

Carex polystachya Sw. ex Wahlenb.

Carex pygmaea Boeckeler

Cyperus aggregatus (Willd.) Endl.

Cyperus articulatus $\mathrm{L}$.

Cyperus chalaranthus J. Presl \& C. Presl

Cyperus distans L. $\mathrm{f}$.

Cyperus friburgensis Boeckeler

Cyperus giganteus Vahl

Cyperus hermaphroditus (Jacq.) Standl.

Cyperus hoppiifolius Uittien

Cyperus laetus J. Presl \& C. Presl

Cyperus laevigatus L.

Cyperus ligularis $\mathrm{L}$.

Cyperus luzulae (L.) Rottb. ex Retz.

Cyperus manimae Kunth

Cyperus miliifolius Poepp. \& Kunth

Cyperus sphacelatus Rottb.

Cyperus surinamensis Rottb.

Cyperus tabina Steud. ex Boeckeler

Cyperus tacnensis Nees \& Meyen

Diplasia karatifolia Rich.

Eleocharis albibracteata Nees \& Meyen ex Kunth

Eleocharis elegans (Kunth) Roem. \& Schult.

Eleocharis montana (Kunth) Roem. \& Schult.

Fimbristylis dichotoma (L.) Vahl

Fimbristylis littoralis Gaudich.

Fuirena incompleta Nees

Isolepis cernua (Vahl) Roem. \& Schult.

Isolepis inundata $\mathrm{R}$. $\mathrm{Br}$.

Kyllinga brevifolia Rottb.

Kyllinga odorata Vahl

Kyllinga pumila Michx.

Oreobolopsis tepalifera T. Koyama \& Guagl.

Oreobolus ecuadorensis T. Koyama

Oxycaryum cubense (Poepp. \& Kunth) Palla

Phylloscirpus acaulis (Phil.) Goetgh. \& D.A. Simpson

Phylloscirpus boliviensis (Barros) Dhooge \& Goetgh.

Phylloscirpus deserticola (Phil.) Dhooge \& Goetgh.

Pycreus bipartitus (Torr.) C.B. Clarke

Pycreus niger (Ruiz \& Pav.) Cufod.

Pycreus unioloides (R. Br.) Urb.

Rhynchospora blepharophora (J. Presl \& C. Presl)

H. Pfeiff.

Rhynchospora dissitiflora Steud. ex Boeckeler

Rhynchospora globosa (Kunth) Roem. \& Schult.
Monnina ruiziana Chodat

Monnina salicifolia Ruiz \& Pav.

Moutabea aculeata (Ruiz \& Pav.) Poepp. \& Endl.

Polygala coridifolia C. Presl

Polygala nemoralis A.W. Benn.

Polygala paniculata L.

Polygala pearcei A.W. Benn.

Pteromonnina herbacea (DC.) B. Eriksen

Securidaca diversifolia (L.) S.F. Blake

Securidaca dolod B. Walln.

Securidaca volubilis $\mathrm{L}$.

\section{Polygonaceae}

Coccoloba acuminata Kunth

Coccoloba densifrons Mart. ex Meisn.

Coccoloba nutans Kunth

Muehlenbeckia tamnifolia (Kunth) Meisn.

Muehlenbeckia volcanica (Benth.) Endl.

Persicaria hydropiperoides (Michx.) Small

Polygonum acuminatum Kunth

Rumex acetosella $\mathrm{L}$.

Rumex conglomeratus Murray

Rumex crispus L.

Rumex obtusifolius L.

Rumex peruanus Rech. f.

Triplaris americana $\mathrm{L}$.

Triplaris poeppigiana Wedd.

\section{Polypodiaceae}

Campyloneurum aglaolepis (Alston) de la Sota

Campyloneurum angustifolium (Sw.) Fée

Campyloneurum angustipaleatum (Alston) M. Mey. ex

Lellinger

Campyloneurum aphanophlebium (Kunze) T. Moore

Campyloneurum asplundii (C. Chr.) Ching

Campyloneurum brevifolium (Lodd. ex Link) Link

Campyloneurum chlorolepis Alston

Campyloneurum fuscosquamatum Lellinger

Campyloneurum phyllitidis (L.) C. Presl

Campyloneurum repens (Aubl.) C. Presl

Campyloneurum vulpinum (Lindm.) Ching

Dicranoglossum desvauxii (Klotzsch) Proctor

Grammitis anfractuosa (Kunze ex Klotzsch) Proctor

Grammitis xiphopteroides (Liebm.) A.R. Sm.

Melpomene flabelliformis (Poir.) A.R. Sm. \& R.C.

Moran

Microgramma baldwinii Brade

Microgramma dictyophylla (Kunze ex Mett.) de la Sota

Microgramma latevagans (Maxon \& C. Chr.) Lellinger

Microgramma percussa (Cav.) de la Sota

Microgramma reptans (Cav.) A.R. Sm.

Microgramma tecta (Kaulf.) Alston 
Rhynchospora locuples C.B. Clarke Rhynchospora macrochaeta Steud. ex Boeckeler Rhynchospora nervosa (Vahl) Boeckeler Rhynchospora polyphylla (Vahl) Vahl Rhynchospora polystachys (Turrill) H. Pfeiff. Rhynchospora rugosa (Vahl) Gale Rhynchospora ruiziana Boeckeler Rhynchospora schiedeana Kunth Rhynchospora vulcani Boeckeler Schoenoplectus californicus (C.A. Mey.) Soják Scirpus fragrans Ruiz \& Pav. Scleria bracteata Cav.

Scleria gaertneri Raddi

Scleria huberi C.B. Clarke

Scleria latifolia Sw.

Scleria macrogyne C.B. Clarke

Scleria macrophylla J. Presl \& C. Presl

Scleria martii (Nees) Steud.

Scleria microcarpa Nees ex Kunth

Scleria stipularis Nees

Uncinia hamata (Sw.) Urb.

Uncinia macrolepis Decne.

Uncinia phleoides (Cav.) Pers.

Zameioscirpus muticus Dhooge \& Goetgh.

\section{Davalliaceae}

Nephrolepis biserrata (Sw.) Schott

Nephrolepis cordifolia (L.) C. Presl

Nephrolepis pectinata (Willd.) Schott

Nephrolepis rivularis (Vahl) Mett. ex Krug

Nephrolepis undulata (Afzel. ex Sw.) J. Sm.

\section{Dennstaedtiaceae}

Blotiella lindeniana (Hook.) R.M. Tryon

Dennstaedtia arborescens (Willd.) Ekman ex Maxon

Dennstaedtia bipinnata (Cav.) Maxon

Dennstaedtia cicutaria (Sw.) T. Moore

Dennstaedtia dissecta (Sw.) T. Moore

Dennstaedtia obtusifolia (Willd.) T. Moore

Dennstaedtia sprucei T. Moore

Dennstaedtia wercklei (Christ) R.M. Tryon

Histiopteris incisa (Thunb.) J. Sm.

Hypolepis repens (L.) C. Presl

Pteridium aquilinum (L.) Kuhn

Pteridium arachnoideum (Kaulf.) Maxon

Pteridium caudatum (L.) Maxon

\section{Dichapetalaceae}

Stephanopodium peruvianum Poepp. \& Endl.

Tapura acreana (Ule) Rizzini

Tapura coriacea J.F. Macbr.

\section{Dicksoniaceae}

Dicksonia sellowiana Hook.
Niphidium albopunctatissimum Lellinger

Niphidium anocarpos (Kunze) Lellinger

Niphidium crassifolium (L.) Lellinger

Niphidium longifolium (Cav.) C.V. Morton \& Lellinger

Niphidium macbridei Lellinger

Phlebodium aureum (L.) J. Sm.

Pleopeltis astrolepis (Liebm.) E. Fourn.

Pleopeltis macrocarpa (Bory ex Willd.) Kaulf.

Polypodium adnatum Kunze ex Klotzsch

Serpocaulon dasypleuron (Kunze) A.R. Sm.

Serpocaulon loriceum (L.) A.R. Sm.

Serpocaulon triseriale (Sw.) A.R. Sm.

Terpsichore gradata (Baker) A.R. Sm.

\section{Pontederiaceae}

Eichhornia crassipes (Mart.) Solms

\section{Portulacaceae}

Portulaca elongata Rusby

Portulaca macbridei D. Legrand

Portulaca oleracea L.

\section{Potamogetonaceae}

Potamogeton illinoensis Morong

Stuckenia filiformis (Pers.) Börner

Zannichellia palustris L.

\section{Primulaceae}

Anagallis arvensis $\mathrm{L}$.

Ardisia albovirens $\mathrm{Mez}$

Ardisia huallagae $\mathrm{Mez}$

Ardisia loretensis Lundell

Ardisia nigrovirens J.F. Macbr.

Ardisia premontana Pipoly

Clavija elliptica Mez

Clavija hookeri A. DC.

Clavija longifolia Ruiz \& Pav.

Clavija macrocarpa Ruiz \& Pav.

Clavija obtusifolia B. Ståhl

Clavija poeppigii Mez

Clavija tarapotana $\mathrm{Mez}$

Clavija weberbaueri Mez

Cybianthus laetus (Mez) G. Agostini

Cybianthus minutiflorus $\mathrm{Mez}$

Cybianthus peruvianus (A. DC.) Miq.

Cybianthus spicatus (Kunth) G. Agostini

Cybianthus venezuelanus Mez

Geissanthus abditus J.F. Macbr.

Geissanthus bolivianus Britton

Geissanthus peruvianus (A. DC.) Mez

Myrsine coriacea (Sw.) R. Br. ex Roem. \& Schult.

Myrsine dependens (Ruiz \& Pav.) Spreng.

Myrsine fosteri Pipoly

Myrsine latifolia (Ruiz \& Pav.) Spreng. 
Lophosoria quadripinnata (J.F. Gmel.) C. Chr.

Dilleniaceae

Curatella americana L.

Doliocarpus dentatus (Aubl.) Standl.

Tetracera parviflora (Rusby) Sleumer

\section{Dioscoreaceae}

Dioscorea acanthogene Rusby

Dioscorea alata L.

Dioscorea altissima Lam.

Dioscorea arifolia C. Presl

Dioscorea chagllaensis R. Knuth

Dioscorea coriacea Humb. \& Bonpl. ex Willd.

Dioscorea decorticans C. Presl

Dioscorea dodecaneura Vell.

Dioscorea iquitosensis R. Knuth

Dioscorea macbrideana R. Knuth

Dioscorea mitoensis R. Knuth

Dioscorea nicolasensis R. Knuth

Dioscorea piperifolia Humb. \& Bonpl. ex Willd.

Dioscorea polygonoides Humb. \& Bonpl. ex Willd.

Dioscorea schunkei Ayala \& T. Clayton

Dioscorea syringifolia (Kunth) Kunth \& R.H. Schomb.

ex R. Knuth

Dioscorea trifida L. f.

\section{Dipentodontaceae}

Perrottetia gentryi Lundell

\section{Dryopteridaceae}

Arachniodes denticulata (Sw.) Ching

Bolbitis hastata (E. Fourn.) Hennipman

Bolbitis lindigii (Mett.) C. Chr.

Bolbitis oligarchica (Baker) Hennipman

Bolbitis semipinnatifida (Fée) Alston

Cyclodium meniscioides (Willd.) C. Presl

Didymochlaena truncatula (Sw.) J. Sm.

Elaphoglossum apodum (Kaulf.) Schott ex J. Sm.

Elaphoglossum craspedotum Copel.

Elaphoglossum cuspidatum (Willd.) T. Moore

Elaphoglossum huacsaro (Ruiz) Christ

Elaphoglossum hystrix (Kunze) T. Moore

Elaphoglossum leprosum (Kuhn) Christ

Elaphoglossum lingua (C. Presl) Brack.

Elaphoglossum nigrescens (Hook.) T. Moore ex

Diels

Elaphoglossum peltatum (Sw.) Urb.

Elaphoglossum raywaense (Jenman) Alston

Elaphoglossum tectum (Humb. \& Bonpl. ex Willd.) T. Moore

Elaphoglossum zebrinum Mickel

Mickelia guianensis (Aubl.) R.C. Moran, Sundue \& Labiak
Myrsine pellucida (Ruiz \& Pav.) Spreng.

Myrsine sessiliflora (Mez) Pipoly

Myrsine weberbaueri (Mez) Pipoly

Parathesis adenanthera (Miq.) Hook. f. ex Mez

Parathesis candolleana Mez

Stylogyne ardisioides (Kunth) Mez

Stylogyne micrantha (Kunth) Mez

Stylogyne nigricans (A. DC.) Mez

\section{Proteaceae}

Euplassa pinnata (Lam.) I.M. Johnst.

Oreocallis grandiflora (Lam.) R. Br.

Roupala monosperma (Ruiz \& Pav.) I.M. Johnst.

\section{Pteridaceae}

Adiantum anceps Maxon \& C.V. Morton

Adiantum cajennense Willd. ex Klotzsch

Adiantum digitatum Hook.

Adiantum latifolium Lam.

Adiantum macrocladum Klotzsch

Adiantum macrophyllum Sw.

Adiantum obliquum Willd.

Adiantum petiolatum Desv.

Adiantum pulverulentum $\mathrm{L}$.

Adiantum scalare R.M. Tryon

Adiantum subvolubile Mett. ex Kuhn

Adiantum tetraphyllum Humb. \& Bonpl. ex Willd.

Adiantum tomentosum Klotzsch

Adiantum villosissimum Mett. ex Kuhn

Ananthacorus angustifolius (Sw.) Underw. \& Maxon

Anetium citrifolium (L.) Splitg.

Argyrochosma stuebeliana (Hieron.) Windham

Astrolepis sinuata (Lag. ex Sw.) D.M. Benham \&

Windham

Cheilanthes bonariensis (Willd.) Proctor

Cheilanthes farinosa (Forssk.) Kaulf.

Cheilanthes myriophylla Desv.

Cheilanthes scariosa (Sw.) C. Presl

Eriosorus flexuosus (Kunth) Copel.

Eriosorus glaberrimus (Maxon) Scamman

Hecistopteris pumila (Spreng.) J. Sm.

Jamesonia cheilanthoides (Sw.) Christenh.

Notholaena sulphurea (Cav.) J. Sm.

Pellaea ovata (Desv.) Weath.

Pellaea ternifolia (Cav.) Link

Pityrogramma calomelanos (L.) Link

Pityrogramma ebenea (L.) Proctor

Pityrogramma trifoliata (L.) R.M. Tryon

Polytaenium lineatum (Sw.) J. Sm. 
Olfersia cervina (L.) Kunze

Polybotrya caudata Kunze

Polybotrya fractiserialis (Baker) J. Sm.

Polystichum nudicaule Rosenst.

Stigmatopteris rotundata (Willd.) C. Chr.

\section{Ebenaceae}

Diospyros artanthifolia Mart. ex Miq.

Diospyros panguana B. Walln.

Diospyros tessmannii Mildbr.

Lissocarpa uyat B. Walln.

\section{Elaeocarpaceae}

Sloanea fragrans Rusby

Sloanea guianensis (Aubl.) Benth.

Sloanea laxiflora Spruce ex Benth.

Sloanea spathulata Earle Sm.

Vallea stipularis L. f.

\section{Elatinaceae}

Elatine peruviana Baehni \& J.F. Macbr.

\section{Ephedraceae}

Ephedra americana Humb. \& Bonpl. ex Willd.

\section{Ericaceae}

Bejaria aestuans Mutis ex L.

Cavendishia bracteata (Ruiz \& Pav. ex J. St.-Hil.)

Hoerold

Cavendishia complectens Hemsl.

Cavendishia isernii Sleumer

Cavendishia nobilis Lindl.

Cavendishia punctata (Ruiz \& Pav. ex J. St.-Hil.)

Sleumer

Cavendishia sirensis Luteyn

Disterigma acuminatum (Kunth) Nied.

Disterigma alaternoides (Kunth) Nied.

Gaultheria bracteata G. Don

Gaultheria buxifolia Willd.

Gaultheria erecta Vent.

Gaultheria foliolosa Benth.

Gaultheria glomerata (Cav.) Sleumer

Gaultheria reticulata Kunth

Gaultheria strigosa Benth.

Gaultheria vaccinioides Wedd.

Macleania benthamiana Walp.

Orthaea ferreyrae A.C. Sm.

Orthaea secundiflora (Poepp. \& Endl.) Klotzsch

Pellegrinia coccinea (Hoerold) Sleumer

Pellegrinia grandiflora (Ruiz \& Pav. ex G. Don)

Sleumer

Pellegrinia harmsiana (Hoerold) Sleumer

Pellegrinia hirsuta (Ruiz \& Pav. ex G. Don) Sleumer

Pernettya prostrata (Cav.) DC.
Pteris altissima Poir.

Pteris biaurita L.

Pteris grandifolia L.

Pteris livida Mett.

Pteris pungens Willd.

Pteris quadriaurita Retz.

Vittaria graminifolia Kaulf.

Vittaria lineata (L.) Sm.

\section{Putranjivaceae}

Drypetes amazonica Steyerm.

\section{Ranunculaceae}

Anemone helleborifolia DC.

Clematis dioica $\mathrm{L}$.

Clematis haenkeana C. Presl

Clematis peruviana DC.

Krapfia macropetala (DC.) Tamura

Oreithales integrifolia (DC.) Schltdl.

Ranunculus breviscapus DC.

Ranunculus chilensis DC.

Ranunculus clypeatus (Ulbr.) Lourteig

Ranunculus flagelliformis Sm.

Ranunculus geranioides Humb., Bonpl. \& Kunth ex DC.

Ranunculus gusmannii Humb. ex Caldas

Ranunculus haemanthus Ulbr.

Ranunculus krapfia DC. ex Deless.

Ranunculus limoselloides Turcz.

Ranunculus luxurians Lourteig

Ranunculus macropetalus DC.

Ranunculus praemorsus Humb., Bonpl. \& Kunth ex DC.

Ranunculus trichophyllus Chaix ex Vill.

Ranunculus weberbaueri (Ulbr.) Lourteig

Thalictrum decipiens B. Boivin

Thalictrum podocarpum Kunth ex DC.

\section{Rhamnaceae}

Colubrina glandulosa Perkins

Condalia weberbaueri Perkins

Frangula sphaerosperma (Sw.) Kartesz \& Gandhi

Gouania latifolia Reissek

Gouania lupuloides (L.) Urb.

Gouania polygama (Jacq.) Urb.

Retanilla ephedra (Vent.) Brongn.

Rhamnidium elaeocarpum Reissek

Rhamnus granulosa (Ruiz \& Pav.) Weberb. ex M.C. Johnst.

\section{Rosaceae}

Acaena cylindristachya Ruiz \& Pav.

Acaena ovalifolia Ruiz \& Pav. 
Psammisia coarctata (Ruiz \& Pav.) A.C. Sm.

Psammisia guianensis Klotzsch

Psammisia ulbrichiana Hoerold

Semiramisia speciosa (Benth.) Klotzsch

Sphyrospermum buxifolium Poepp. \& Endl.

Themistoclesia peruviana A.C. Sm.

Thibaudia biflora (Poepp. \& Endl.) Hoerold

Thibaudia cardiophylla Sleumer

Thibaudia crenulata J. Rémy

Thibaudia croatii Luteyn

Thibaudia floribunda Kunth

Thibaudia harmsiana Hoerold

Thibaudia mellifera Ruiz \& Pavon ex St. Hil.

Thibaudia moricandii Dunal

Thibaudia uniflora A.C. Sm.

Vaccinium corymbodendron Ruiz \& Pav. ex Dunal

Vaccinium dependens (G. Don) Sleumer

Vaccinium floribundum Kunth

\section{Eriocaulaceae}

Paepalanthus ensifolius (Kunth) Kunth

Paepalanthus pilosus (Kunth) Kunth

Paepalanthus planifolius (Bong.) Körn.

\section{Erythroxylaceae}

Erythroxylum coca Lam.

Erythroxylum fimbriatum Peyr.

Erythroxylum gracilipes Peyr.

Erythroxylum hondense Kunth

Erythroxylum macrophyllum Cav.

Erythroxylum mamacoca Mart.

Erythroxylum mucronatum Benth.

Erythroxylum novogranatense (D. Morris) Hieron.

Erythroxylum schunkei Plowman

Erythroxylum ulei O.E. Schulz

\section{Escalloniaceae}

Escallonia myrtilloides L. f.

Escallonia paniculata (Ruiz \& Pav.) Roem. \& Schult.

Escallonia pendula (Ruiz \& Pav.) Pers.

Escallonia resinosa (Ruiz \& Pav.) Pers.

\section{Euphorbiaceae}

Acalypha cuneata Poepp.

Acalypha diversifolia Jacq.

Acalypha infesta Poepp.

Acalypha macrostachya Jacq.

Acalypha peruviana Müll. Arg.

Acalypha plicata Müll. Arg.

Acalypha ruiziana Müll. Arg.

Acalypha salicifolia Müll. Arg.

Acalypha scandens Benth.

Acalypha stachyura Pax

Acalypha stenoloba Müll. Arg.
Alchemilla andina (L.M. Perry) J.F. Macbr.

Geum peruvianum Focke

Hesperomeles cuneata Lindl.

Hesperomeles ferruginea (Pers.) Benth.

Hesperomeles gayana (Decne.) J.F. Macbr.

Hesperomeles obtusifolia (Pers.) Lindl.

Hesperomeles weberbaueri C.K. Schneid.

Kageneckia lanceolata Ruiz \& Pav.

Lachemilla aphanoides (Mutis ex L. f.) Rothm.

Lachemilla diplophylla (Diels) Rothm.

Lachemilla erodiifolia (Wedd.) Rothm.

Lachemilla galioides (Benth.) Rothm.

Lachemilla nivalis (Kunth) Rothm.

Lachemilla orbiculata (Ruiz \& Pav.) Rydb.

Lachemilla pinnata (Ruiz \& Pav.) Rothm.

Lachemilla procumbens (Rose) Rydb.

Margyricarpus pinnatus (Lam.) Kuntze

Polylepis besseri Hieron.

Polylepis pauta Hieron.

Polylepis racemosa Ruiz \& Pav.

Potentilla dombeyi Nestl.

Prunus amplifolia Pilg.

Prunus debilis Koehne

Prunus huantensis Pilg.

Prunus integrifolia (C. Presl) Walp.

Prunus oblonga J.F. Macbr.

Prunus ovalis Ruiz ex Koehne

Prunus persica (L.) Batsch

Prunus pleiantha Pilg.

Prunus ruiziana Koehne

Prunus serotina Ehrh.

Rubus acanthophyllos Focke

Rubus adenothallus Focke

Rubus bogotensis Kunth

Rubus floribundus Weihe

Rubus glaucus Benth.

Rubus hedycarpus Focke

Rubus megalococcus Focke

Rubus nubigenus Kunth

Rubus roseus Poir.

Rubus sparsiflorus J.F. Macbr.

Rubus urticifolius Poir.

Rubus weberbaueri Focke

\section{Rubiaceae}

Alibertia bertierifolia K. Schum.

Alibertia curviflora K. Schum.

Arachnothryx tenuisepala Borhidi

Arcytophyllum filiforme (Ruiz \& Pav.) Standl.

Arcytophyllum setosum (Ruiz \& Pav.) Schltdl.

Arcytophyllum thymifolium (Ruiz \& Pav.) Standl. 
Acalypha stricta Poepp. \& Endl.

Acidoton nicaraguensis (Hemsl.) G.L. Webster

Actinostemon amazonicus Pax \& K. Hoffm.

Actinostemon imbricatus Müll. Arg.

Alchornea castaneifolia (Humb. \& Bonpl. ex Willd.)

A. Juss.

Alchornea glandulosa Poepp.

Alchornea latifolia Sw.

Alchorneopsis floribunda (Benth.) Müll. Arg.

Bia fallax (Müll. Arg.) G.L. Webster

Chamaesyce ruiziana (Klotzsch \& Garcke) G.L.

Webster

Conceveiba rhytidocarpa Müll. Arg.

Croton balsameus Müll. Arg.

Croton erythrochilus Müll. Arg.

Croton fragrantulus Croizat

Croton glandulosus $\mathrm{L}$.

Croton lechleri Müll. Arg.

Croton matourensis Aubl.

Croton palanostigma Klotzsch

Croton sampatik Müll. Arg.

Croton sapiiflorus Croizat

Croton tessmannii Mansf.

Dalechampia aristolochiifolia Kunth

Dalechampia cissifolia Poepp.

Dalechampia dioscoreifolia Poepp.

Dalechampia tiliifolia Lam.

Euphorbia heterophylla L.

Euphorbia hirta L.

Euphorbia peplus L.

Euphorbia viridis (Klotzsch \& Garcke) Boiss.

Hevea brasiliensis (Willd. ex A. Juss.) Müll. Arg.

Hevea guianensis Aubl.

Hura crepitans L.

Jatropha macrantha Müll. Arg.

Jatropha weberbaueri Pax \& K. Hoffm.

Mabea occidentalis Benth.

Mabea standleyi Steyerm.

Manihot esculenta Crantz

Manihot leptophylla Pax \& K. Hoffm.

Maprounea guianensis Aubl.

Nealchornea yapurensis Huber

Pausandra trianae (Müll. Arg.) Baill.

Plukenetia brachybotrya Müll. Arg.

Pseudosenefeldera inclinata (Müll. Arg.) Esser

Ricinus communis $\mathrm{L}$.

Sapium glandulosum (L.) Morong

Sapium marmieri Huber

Sebastiania obtusifolia Pax \& K. Hoffm.

Tetrorchidium macrophyllum Müll. Arg.
Bertiera guianensis Aubl.

Borreria capitata (Ruiz \& Pav.) DC.

Borreria tenella (Kunth) Cham. \& Schltdl.

Calycophyllum megistocaulum (K. Krause) C.M.

Taylor

Calycophyllum spruceanum (Benth.) Hook. f. ex K.

Schum.

Chimarrhis hookeri K. Schum.

Chiococca alba (L.) Hitchc.

Chomelia paniculata (Bartl. ex DC.) Steyerm.

Chomelia spinosa Jacq.

Cinchona glandulifera (Ruiz) Ruiz \& Pav.

Cinchona hirsuta Ruiz \& Pav.

Cinchona micrantha Ruiz \& Pav.

Cinchona nitida Ruiz \& Pav.

Cinchona officinalis $\mathrm{L}$.

Cinchona pubescens Vahl

Cinchona pyrifolia L. Andersson

Cinchona villosa Pav. ex Lindl.

Coccocypselum condalia Pers.

Coccocypselum hirsutum Bartl. ex DC.

Coccocypselum lanceolatum (Ruiz \& Pav.) Pers.

Coffea arabica $\mathrm{L}$.

Coffea canephora Pierre ex A. Froehner

Condaminea corymbosa (Ruiz \& Pav.) DC.

Condaminea glabrata Bartl. ex DC.

Condaminea microcarpa (Ruiz \& Pav.) DC.

Cosmibuena grandiflora (Ruiz \& Pav.) Rusby

Coussarea acuminata (Ruiz \& Pav.) Zappi

Coussarea albescens (DC.) Müll. Arg.

Coussarea hirticalyx Standl.

Coussarea megalocarpa Standl.

Coussarea obliqua Standl.

Coussarea paniculata (Willd.) Standl.

Coussarea platyphylla Müll. Arg.

Coussarea rudgeoides Rusby

Coutarea hexandra (Jacq.) K. Schum.

Duroia hirsuta (Poepp.) K. Schum.

Elaeagia karstenii Standl.

Emmeorhiza umbellata (Spreng.) K. Schum.

Exostema corymbosum (Ruiz \& Pav.) Spreng.

Faramea anisocalyx Poepp. \& Endl.

Faramea capillipes Müll. Arg.

Faramea exemplaris Standl.

Faramea glandulosa Poepp.

Faramea miconioides Standl.

Faramea multiflora A. Rich. ex DC.

Faramea quinqueflora Poepp.

Faramea subsessilis (Ruiz \& Pav.) Standl.

Galianthe peruviana (Pers.) E.L. Cabral 
Tetrorchidium rubrivenium Poepp.

Tragia subhastata Poepp.

\section{Fabaceae}

Adesmia hispidula (Lag.) DC.

Aeschynomene americana L.

Albizia carbonaria Britton

Albizia niopoides (Spruce ex Benth.) Burkart

Albizia pedicellaris (DC.) L. Rico

Amburana cearensis (Allemão) A.C. Sm.

Amicia lobbiana Benth. ex Rusby

Anadenanthera colubrina (Vell.) Brenan

Andira inermis (W. Wright) Kunth ex DC.

Andira multistipula Ducke

Apurimacia boliviana (Britton) Lavin

Astragalus garbancillo Cav.

Astragalus pickeringii A. Gray

Barbieria pinnata (Pers.) Baill.

Bauhinia aculeata L.

Bauhinia glabra Jacq.

Bauhinia guianensis Aubl.

Bauhinia longicuspis Spruce ex Benth.

Bauhinia tarapotensis Benth.

Bauhinia weberbaueri Harms

Browneopsis excelsa Pittier

Caesalpinia cassioides Willd.

Caesalpinia decapetala (Roth) Alston

Cajanus cajan (L.) Huth

Calliandra angustifolia Spruce ex Benth.

Calliandra taxifolia (Kunth) Benth.

Calliandra trinervia Benth.

Calopogonium caeruleum (Benth.) C. Wright

Canavalia brasiliensis Mart. ex Benth.

Canavalia ensiformis (L.) DC.

Canavalia eurycarpa Piper

Canavalia glabra (M. Martens \& Galeotti) J.D. Sauer

Canavalia sericophylla Ducke

Cassia grandis L. f.

Cedrelinga cateniformis (Ducke) Ducke

Centrosema plumieri (Turpin ex Pers.) Benth

Centrosema triquetrum (Hoffmanns. ex Benth.)

Benth.

Chaetocalyx klugii Rudd

Chamaecrista glandulosa (L.) Greene

Clitoria flexuosa Fantz

Clitoria pozuzoensis J.F. Macbr.

Cojoba arborea (L.) Britton \& Rose

Cojoba chazutensis (Standl.) L. Rico

Cojoba sophorocarpa (Benth.) Britton \& Rose

Collaea speciosa (Loisel.) DC.
Galium corymbosum Ruiz \& Pav.

Galium hypocarpium (L.) Endl. ex Griseb.

Galium obovatum Kunth

Galium pumilio Standl.

Galium weberbaueri Krause

Genipa americana L.

Geophila cordifolia Miq.

Geophila macropoda (Ruiz \& Pav.) DC.

Geophila repens (L.) I.M. Johnst.

Gonzalagunia dependens Ruiz \& Pav.

Gonzalagunia mildrediae D.R. Simpson ex C.M.

Taylor

Guettarda aromatica Poepp. \& Endl.

Guettarda crispiflora Vahl

Guettarda dependens (Ruiz \& Pav.) DC.

Guettarda pohliana Müll. Arg.

Hamelia axillaris Sw.

Hamelia patens Jacq.

Hillia illustris (Vell.) K. Schum.

Hillia macrophylla Standl.

Hillia parasitica Jacq.

Hippotis triflora Ruiz \& Pav.

Hoffmannia aggregata (Ruiz \& Pav.) K. Schum.

Hoffmannia latifolia (Bartl. ex DC.) Kuntze

Hoffmannia obovata (Ruiz \& Pav.) Standl.

Hoffmannia verticillata (Ruiz \& Pav.) Standl.

Hoffmannia villosula Standl.

Isertia laevis (Triana) B.M. Boom

Ixora acuminatissima Müll. Arg.

Joosia dichotoma (Ruiz \& Pav.) H. Karst.

Joosia pulcherrima Steere

Joosia umbellifera $\mathrm{H}$. Karst.

Ladenbergia acutifolia (Ruiz \& Pav.) Klotzsch

Ladenbergia oblongifolia (Humb. ex Mutis) L.

Andersson

Macbrideina peruviana Standl.

Macrocnemum roseum (Ruiz \& Pav.) Wedd.

Malanea boliviana Standl.

Manettia acutifolia Ruiz \& Pav.

Manettia cordifolia Mart.

Manettia hispida Poepp. \& Endl.

Manettia leucantha K. Krause

Manettia modica Standl.

Manettia paniculata Poepp. \& Endl.

Manettia peruviana Standl.

Manettia racemosa Ruiz \& Pav.

Manettia reclinata $\mathrm{L}$.

Manettia umbellata Ruiz \& Pav.

Margaritopsis boliviana (Standl.) C.M. Taylor

Margaritopsis nana (K. Krause) C.M. Taylor 
Cologania broussonetii (Balb.) DC.

Copaifera reticulata Ducke

Coursetia fruticosa (Cav.) J.F. Macbr.

Crotalaria incana L.

Crotalaria maypurensis Kunth

Crotalaria nitens Kunth

Crotalaria pallida Aiton

Crotalaria pumila Ortega

Crotalaria sagittalis L.

Crotalaria trichotoma Bojer

Crudia glaberrima (Steud.) J.F. Macbr.

Dalbergia frutescens (Vell.) Britton

Dalea coerulea (L. f.) Schinz \& Thell.

Dalea cylindrica Hook.

Dalea exilis DC.

Dalea galbina (J.F. Macbr.) J.F. Macbr.

Desmodium adscendens (Sw.) DC.

Desmodium axillare (Sw.) DC.

Desmodium cajanifolium (Kunth) DC.

Desmodium campyloclados Hemsl.

Desmodium distortum (Aubl.) J.F. Macbr.

Desmodium intortum (Mill.) Urb.

Desmodium limense Hook.

Desmodium molliculum (Kunth) DC.

Desmodium neomexicanum A. Gray

Desmodium purpusii Brandegee

Desmodium subsericeum Malme

Desmodium tortuosum (Sw.) DC.

Desmodium uncinatum (Jacq.) DC.

Desmodium vargasianum B.G. Schub.

Dioclea funalis Poepp.

Dioclea virgata (Rich.) Amshoff

Dipteryx alata Vogel

Erythrina edulis Triana ex Micheli

Erythrina poeppigiana (Walp.) O.F. Cook

Erythrina rubrinervia Kunth

Erythrina ulei Harms

Galactia augusti Harms

Galactia striata (Jacq.) Urb.

Glycine max (L.) Merr.

Indigofera hendecaphylla Jacq.

Indigofera parodiana Burkart

Indigofera suffruticosa Mill.

Indigofera trita L. f.

Indigofera truxillensis Kunth

Inga adenophylla Pittier

Inga alba (Sw.) Willd.

Inga augustii Harms

Inga capitata Desv.
Mitracarpus hirtus (L.) DC.

Nertera granadensis (Mutis ex L. f.) Druce

Notopleura epiphytica (K. Krause) C.M. Taylor

Notopleura iridescens C.M. Taylor

Notopleura macrophylla (Ruiz \& Pav.) C.M. Taylor

Paederia brasiliensis (Hook. f.) Puff

Pagamea dudleyi Steyerm.

Palicourea amethystina (Ruiz \& Pav.) DC.

Palicourea aphthosa Standl.

Palicourea cuspidata (Bredem. ex Schult.) C.M.

Taylor

Palicourea cymosa (Ruiz \& Pav.) Standl.

Palicourea guianensis Aubl.

Palicourea kanehirae Standl.

Palicourea lasiantha K. Krause

Palicourea latifolia K. Krause

Palicourea laxa Roem. \& Schult.

Palicourea macbridei Standl.

Palicourea macrobotrys (Ruiz \& Pav.) DC.

Palicourea mansoana (Müll. Arg.) Standl.

Palicourea microcarpa (Ruiz \& Pav.) Zappi

Palicourea mitis (Ruiz \& Pav.) DC.

Palicourea obovata (Ruiz \& Pav.) DC.

Palicourea punicea (Ruiz \& Pav.) DC.

Palicourea subscandens Standl. ex Steyerm.

Palicourea subtomentosa (Ruiz \& Pav.) C.M. Taylor

Palicourea sulphurea (Ruiz \& Pav.) DC.

Palicourea thyrsiflora (Ruiz \& Pav.) DC.

Palicourea triphylla DC.

Palicourea weberbaueri K. Krause

Pentagonia amazonica (Ducke) L. Andersson \& Rova

Pentagonia spathicalyx K. Schum.

Pentagonia williamsii Standl.

Psychotria abdita Standl.

Psychotria brachiata Sw.

Psychotria bracteocardia (DC.) Müll. Arg.

Psychotria buchtienii (H.J.P. Winkl.) Standl.

Psychotria capitata Ruiz \& Pav.

Psychotria carthagenensis Jacq.

Psychotria conephoroides (Rusby) C.M. Taylor

Psychotria dudleyi Steyerm.

Psychotria ernestii K. Krause

Psychotria hoffmannseggiana (Schult.) Müll. Arg.

Psychotria lupulina Benth.

Psychotria marginata Sw.

Psychotria microbotrys Ruiz ex Standl.

Psychotria ostreophora (Wernham) C.M. Taylor

Psychotria pilosa Ruiz \& Pav.

Psychotria racemosa Rich.

Psychotria repanda Ruiz \& Pav. 
Inga cayennensis Sagot ex Benth.

Inga densiflora Benth.

Inga feuillei DC.

Inga lallensis Spruce ex Benth.

Inga lineata Benth.

Inga marginata Willd.

Inga nobilis Willd.

Inga pavoniana G. Don

Inga pluricarpellata T.D. Penn.

Inga pruriens Poepp.

Inga punctata Willd.

Inga quaternata Poepp.

Inga ruiziana G. Don

Inga semialata (Vell.) Mart.

Inga setosa G. Don

Inga spectabilis (Vahl) Willd.

Inga stipulacea G. Don

Inga tenuistipula Ducke

Inga thibaudiana DC.

Inga tomentosa Benth.

Inga vera Willd.

Inga ynga (Vell.) J.W. Moore

Lathyrus pusillus Elliott

Leucaena leucocephala (Lam.) de Wit

Leucaena trichodes (Jacq.) Benth.

Lonchocarpus hylobius Harms

Lupinus amboensis C.P. Sm.

Lupinus brachypremnon C.P. Sm.

Lupinus chavanillensis (J.F. Macbr.) C.P. Sm.

Lupinus chlorolepis C.P. Sm.

Lupinus condensiflorus C.P. Sm.

Lupinus eanophyllus C.P. Sm.

Lupinus falsomutabilis C.P. Sm.

Lupinus francis-whittieri C.P. Sm.

Lupinus hornemannii J. Agardh

Lupinus huaronensis J.F. Macbr.

Lupinus lindleyanus J. Agardh

Lupinus macbrideanus C.P. Sm.

Lupinus microphyllus Desr.

Lupinus mutabilis Sweet

Lupinus pachitensis C.P. Sm.

Lupinus pickeringii A. Gray

Lupinus praealtus C.P. Sm.

Lupinus xanthophyllus C.P. Sm.

Machaerium cuspidatum Kuhlm. \& Hoehne

Machaerium floribundum Benth.

Machaerium huanucoense Rudd
Psychotria reticulata Ruiz \& Pav.

Psychotria retifera Standl.

Psychotria ruizii Standl.

Psychotria stenostachya Standl.

Psychotria tenuicaulis K. Krause

Psychotria trichotoma M. Martens \& Galeotti

Psychotria trifida Ruiz \& Pav.

Psychotria villosa Ruiz \& Pav.

Psychotria virgata Ruiz \& Pav.

Psychotria viridis Ruiz \& Pav.

Psychotria yapasensis Standl.

Randia armata (Sw.) DC.

Randia obovata Ruiz \& Pav.

Randia tessmannii Standl.

Remijia chelomaphylla G.A. Sullivan

Retiniphyllum fuchsioides K. Krause

Retiniphyllum tepuiense Steyerm.

Ronabea emetica (L. f.) A. Rich.

Ronabea latifolia Aubl.

Rosenbergiodendron longiflorum (Ruiz \& Pav.)

Fagerl.

Rudgea ciliata (Ruiz \& Pav.) Spreng.

Rudgea foveolata (Ruiz \& Pav.) Zahlbr.

Rudgea insolita Standl.

Rudgea palicoureoides (Mart.) Müll. Arg.

Rudgea poeppigii K. Schum. ex Standl.

Rudgea verticillata (Ruiz \& Pav.) Spreng.

Rustia rubra Standl. ex D.R. Simpson

Sabicea cana Hook. f.

Sabicea grisea Cham. \& Schltdl.

Sabicea panamensis Wernham

Sabicea pumila Bartl. ex DC.

Sabicea umbellata (Ruiz \& Pav.) Pers.

Sabicea villosa Schult.

Schizocalyx obovatus (K. Schum. ex Standl.) Kainul.

\& B. Bremer

Schizocalyx peruvianus (K. Krause) Kainul. \& B.

Bremer

Schizocalyx sterculioides (Standl.) Kainul. \& B.

Bremer

Sickingia tinctoria (Aubl.) Lemée

Simira rubescens (Benth.) Bremek. ex Steyerm.

Spermacoce alata Aubl.

Spermacoce exilis (L.O. Williams) C.D. Adams

Spermacoce remota Lam.

Spermacoce tenuior $\mathrm{L}$.

Tocoyena williamsii Standl.

Uncaria guianensis (Aubl.) J.F. Gmel.

Warszewiczia ambigua Standl.

Warszewiczia coccinea (Vahl) Klotzsch

\section{Ruppiaceae}


Macrolobium acaciifolium (Benth.) Benth. Macrolobium gracile Spruce ex Benth. Macrolobium taylorii D.R. Simpson Urb. Macroptilium atropurpureum (Moc. \& Sessé ex DC.)

Macroptilium erythroloma (Mart. ex Benth.) Urb. Macroptilium lathyroides (L.) Urb.

Medicago lupulina L.

Medicago polymorpha $\mathrm{L}$.

Melilotus indicus (L.) All.

Mimosa diplotricha C. Wright ex Sauvalle

Mimosa guilandinae (DC.) Barneby

Mimosa myriadenia (Benth.) Benth.

Mimosa tarda Barneby

Mucuna elliptica (Ruiz \& Pav.) DC.

Mucuna rostrata Benth.

Myroxylon balsamum (L.) Harms

Ormosia schunkei Rudd

Otholobium mexicanum (L. f.) J.W. Grimes

Otholobium munyense (J.F. Macbr.) J.W. Grimes

Otholobium pubescens (Poir.) J.W. Grimes

Paramachaerium schunkei Rudd

Parkia nitida Miq.

Phaseolus augusti Harms

Phaseolus coccineus L.

Phaseolus pachyrrhizoides Harms

Phaseolus vulgaris $\mathrm{L}$.

Piptadenia pteroclada Benth.

Pisum sativum L.

Platymiscium pinnatum (Jacq.) Dugand

Poissonia orbicularis (Benth.) Hauman

Pterocarpus amazonum (Mart. ex Benth.) Amshoff

Pterocarpus rohrii Vahl

Rhynchosia melanocarpa Grear

Rhynchosia phaseoloides (Sw.) DC.

Samanea saman (Jacq.) Merr.

Schizolobium parahyba (Vell.) S.F. Blake

Schnella porphyrotricha (Harms) Wunderlin

Schnella pterocalyx (Ducke) Wunderlin

Senegalia loretensis (J.F. Macbr.) Seigler \& Ebinger

Senegalia macbridei (Britton \& Rose ex J.F. Macbr.)

Seigler \& Ebinger

Senegalia tenuifolia (L.) Britton \& Rose

Senna aurantia (Ruiz \& Pav. ex G. Don) H.S. Irwin \&

Barneby

Senna birostris (Dombey ex Vogel) H.S. Irwin \&

Barneby

Senna cobanensis (Britton) H.S. Irwin \& Barneby

Senna cushina (J.F. Macbr.) H.S. Irwin \& Barneby

Senna latifolia (G. Mey.) H.S. Irwin \& Barneby

Senna macrophylla (Kunth) H.S. Irwin \& Barneby
Ruppia filifolia (Phil.) Skottsb.

\section{Rutaceae}

Dictyoloma vandellianum A. Juss.

Erythrochiton fallax Kallunki

Esenbeckia amazonica Kaastra

Murraya paniculata (L.) Jack

Pilocarpus peruvianus (J.F. Macbr.) Kaastra

Ruta chalepensis L.

Zanthoxylum acuminatum (Sw.) Sw.

Zanthoxylum culantrillo Kunth

Zanthoxylum mantaro (J.F. Macbr.) J.F. Macbr.

Zanthoxylum riedelianum Engl.

\section{Sabiaceae}

Meliosma herbertii Rolfe

Meliosma meridensis Lasser

Meliosma sirensis A.H. Gentry

\section{Saccolomataceae}

Saccoloma elegans Kaulf.

Saccoloma inaequale (Kunze) Mett.

\section{Salicaceae}

Abatia parviflora Ruiz \& Pav.

Abatia rugosa Ruiz \& Pav.

Banara axilliflora Sleumer

Banara guianensis Aubl.

Banara nitida Spruce ex Benth.

Casearia aculeata Jacq.

Casearia arborea (Rich.) Urb.

Casearia commersoniana Cambess.

Casearia decandra Jacq.

Casearia fasciculata (Ruiz \& Pav.) Sleumer

Casearia gossypiosperma Briq.

Casearia mariquitensis Kunth

Casearia maynacarpa Liesner \& P. Jørg.

Casearia nigricans Sleumer

Casearia obovalis Poepp. ex Griseb.

Casearia prunifolia Kunth

Casearia sylvestris Sw.

Casearia ulmifolia Vahl ex Vent.

Hasseltia floribunda Kunth

Laetia procera (Poepp.) Eichler

Lunania parviflora Spruce ex Benth.

Neoptychocarpus killipii (Monach.) Buchheim

Pineda incana Ruiz \& Pav.

Pleuranthodendron lindenii (Turcz.) Sleumer

Prockia crucis P. Browne ex L.

Ryania speciosa Vahl

Salix humboldtiana Willd. 
Senna multijuga (Rich.) H.S. Irwin \& Barneby Senna obliqua (G. Don) H.S. Irwin \& Barneby Senna pendula (Humb. \& Bonpl. ex Willd.) H.S. Irwin \& Barneby Senna reticulata (Willd.) H.S. Irwin \& Barneby Senna ruiziana (G. Don) H.S. Irwin \& Barneby Senna silvestris (Vell.) H.S. Irwin \& Barneby Senna spectabilis (DC.) H.S. Irwin \& Barneby Sesbania exasperata Kunth

Spartium junceum L.

Stryphnodendron guianense (Aubl.) Benth.

Swartzia myrtifolia Sm.

Tachigali formicarum Harms

Tephrosia sinapou (Buc'hoz) A. Chev.

Teramnus uncinatus (L.) Sw.

Trifolium amabile Kunth

Trischidium alternum (Benth.) H.E. Ireland Vachellia aroma (Gillies ex Hook. \& Arn.) Seigler \&

Ebinger

Vachellia macracantha (Humb. \& Bonpl. ex Willd.)

Seigler \& Ebinger

Vicia andicola Kunth

Vicia faba L.

Vigna candida (Vell.) Maréchal, Mascherpa \&

Stainier

Vigna caracalla (L.) Verdc.

Vigna hosei (Craib) Backer

Vigna linearis (Kunth) Maréchal, Mascherpa \&

Stainier

Vigna luteola (Jacq.) Benth.

Vigna unguiculata (L.) Walp.

Vigna vexillata (L.) A. Rich.

Zapoteca amazonica (Benth.) H.M. Hern.

Zapoteca portoricensis (Jacq.) H.M. Hern.

Zygia coccinea (G. Don) L. Rico

Zygia juruana (Harms) L. Rico

Zygia longifolia (Humb. \& Bonpl. ex Willd.) Britton \&

Rose

\section{Gentianaceae}

Chelonanthus alatus (Aubl.) Pulle

Gentiana sedifolia Kunth

Gentianella calanchoides (Gilg) Fabris

Gentianella coccinea (D. Don ex G. Don) Zarucchi

Gentianella dianthoides (Kunth) Fabris ex J.S.

Pringle

Gentianella ericothamna (Gilg) Zarucchi

Gentianella incurva (Hook.) Fabris

Gentianella nitida (Griseb.) Fabris

Gentianella oreosilene (Gilg) J.S. Pringle

Gentianella petrophila (Gilg) Zarucchi

Gentianella pseudolycopodium (Gilg) J.S. Pringle
Tetrathylacium macrophyllum Poepp.

Xylosma intermedia (Seem.) Triana \& Planch.

Xylosma tessmannii Sleumer

\section{Santalaceae}

Antidaphne andina Kuijt

Antidaphne viscoidea Poepp. \& Endl.

Dendrophthora capitellata Rizzini

Dendrophthora chrysostachya (C. Presl) Urb.

Dendrophthora clavata (Benth.) Urb.

Dendrophthora diffusa Kuijt

Dendrophthora ferruginea Patsch.

Dendrophthora lindeniana Tiegh.

Kuijt

Dendrophthora ramosa Patsch.

Dendrophthora virgata (Trel. ex J.F. Macbr.) Kuijt

Phoradendron northropiae Urb.

Phoradendron piperoides (Kunth) Trel.

Phoradendron storkii F.A. Barkley

\section{Sapindaceae}

Allophylus floribundus (Poepp.) Radlk.

Allophylus loretensis Standl. ex J.F. Macbr.

Allophylus paniculatus (Poepp.) Radlk.

Allophylus peruvianus Radlk.

Allophylus pilosus (J.F. Macbr.) A.H. Gentry

Allophylus punctatus (Poepp.) Radlk.

Allophylus scrobiculatus (Poepp.) Radlk.

Cardiospermum corindum $\mathrm{L}$.

Cardiospermum grandiflorum Sw.

Cardiospermum halicacabum L.

Dodonaea viscosa Jacq.

Llagunoa nitida Ruiz \& Pav.

Paullinia acutangula (Ruiz \& Pav.) Pers.

Paullinia alata G. Don

Paullinia bracteosa Radlk.

Paullinia dasystachya Radlk.

Paullinia echinata Huber

Paullinia elegans Cambess.

Paullinia enneaphylla G. Don

Paullinia faginea (Triana \& Planch.) Radlk.

Paullinia fistulosa Radlk.

Paullinia hemiptera D.R. Simpson

Paullinia ingifolia Rich. ex Juss.

Paullinia obovata (Ruiz \& Pav.) Pers.

Paullinia paullinioides Radlk. 
Gentianella ruizii (Griseb.) Holub Gentianella thyrsoidea (Hook.) Fabris Gentianella violacea (D. Don ex G. Don) Fabris Halenia brevicornis (Kunth) G. Don Halenia umbellata (Ruiz \& Pav.) Gilg Halenia weddelliana Gilg Lehmanniella huanucensis Simonis Macrocarpaea corymbosa (Ruiz \& Pav.) Ewan Macrocarpaea kayakifolia J.R. Grant Macrocarpaea obnubilata J.R. Grant Macrocarpaea ostentans J.R. Grant Macrocarpaea ovalis (Ruiz \& Pav.) Ewan Macrocarpaea pachystyla Gilg Macrocarpaea pajonalis J.R. Grant Macrocarpaea tabula-fluctivagifolia J.R. Grant Macrocarpaea viscosa (Ruiz \& Pav.) Gilg Macrocarpaea wallnoeferi J.R. Grant Macrocarpaea weigendiorum J.R. Grant Potalia amara Aubl.

Symbolanthus elisabethae (M.R. Schomb.) Gilg Tachia occidentalis Maguire \& Weaver Tachia parviflora Maguire \& Weaver Voyria tenella Hook. Voyria truncata (Standl.) Standl. \& Steyerm. Voyriella parviflora (Miq.) Miq.

\section{Geraniaceae}

Geranium ayavacense Willd. ex Kunth Geranium chilloense Willd. ex Kunth Geranium fallax Steud. Geranium humboldtii Willd. ex Spreng. Geranium jaekelae J.F. Macbr. Geranium macbridei Aedo Geranium ruizii Hieron. Geranium seemannii Peyr. Geranium sessiliflorum Cav. Geranium sibbaldioides Benth.

\section{Gesneriaceae}

Alloplectus tessmannii Mansf. Alloplectus weirii (Kuntze) Wiehler Amalophyllon divaricatum (Poepp.) Boggan, L.E.

Skog \& Roalson Besleria aggregata (Mart.) Hanst. Besleria angusta C.V. Morton Besleria barbata (Poepp.) Hanst. Besleria capitata Poepp. Besleria ferreyrae C.V. Morton Besleria gracilenta C.V. Morton Besleria hutchisonii C.V. Morton Besleria lucida Poepp. Besleria membranacea C.V. Morton
Paullinia pinnata L.

Paullinia selenoptera Radlk.

Paullinia setosa Radlk.

Paullinia tenera Poepp.

Sapindus saponaria L.

Serjania altissima (Poepp.) Radlk.

Serjania brachyptera Radlk.

Serjania caracasana (Jacq.) Willd.

Serjania communis Cambess.

Serjania deltoidea Radlk.

Serjania dibotrya Poepp.

Serjania diffusa Radlk.

Serjania fuscostriata Radlk.

Serjania glabrata Kunth

Serjania grandifolia Sagot ex Radlk.

Serjania inflata Poepp.

Serjania inscripta Radlk.

Serjania leptocarpa Radlk.

Serjania membranacea Splitg.

Serjania mollis Kunth

Serjania nutans Poepp.

Serjania pyramidata Radlk.

Serjania rubicaulis Benth. ex Radlk.

Serjania sphaerococca Radlk.

Serjania sufferruginea Radlk.

Serjania tenuifolia Radlk.

Talisia cerasina (Benth.) Radlk.

Talisia pinnata (Ruiz \& Pav.) Radlk.

Talisia retusa R.S. Cowan

Thinouia obliqua Radlk.

Toulicia reticulata Radlk.

Urvillea ulmacea Kunth

\section{Sapotaceae}

Chrysophyllum amazonicum T.D. Penn.

Chrysophyllum argenteum Jacq.

Chrysophyllum ovale Rusby

Chrysophyllum prieurii A. DC.

Chrysophyllum sanguinolentum (Pierre) Baehni

Ecclinusa lanceolata (Mart. \& Eichler) Pierre

Manilkara bidentata (A. DC.) A. Chev.

Manilkara inundata (Ducke) Ducke

Micropholis egensis (A. DC.) Pierre

Micropholis guyanensis (A. DC.) Pierre

Micropholis melinoniana Pierre

Micropholis venulosa (Mart. \& Eichler) Pierre

Pouteria ambelaniifolia (Sandwith) T.D. Penn.

Pouteria caimito (Ruiz \& Pav.) Radlk.

Pouteria cuspidata (A. DC.) Baehni

Pouteria durlandii (Standl.) Baehni 
Besleria peruviana Fritsch Besleria placita C.V. Morton Besleria racemosa C.V. Morton Besleria reticulata Fritsch Besleria tetrangularis Ruiz ex Hanst.

Besleria variabilis C.V. Morton Codonanthe crassifolia (H. Focke) C.V. Morton Codonanthe uleana Fritsch Codonanthopsis dissimulata (H.E. Moore) Wiehler Codonanthopsis ulei Mansf. Columnea ericae Mansf. Columnea fuscihirta L.P. Kvist \& L.E. Skog Columnea inaequilatera Poepp.

Columnea moesta Poepp. Columnea peruviana Zahlbr. Columnea purpureovittata (Wiehler) B.D. Morley Columnea xiphoidea J.F. Sm. \& L.E. Skog Corytoplectus speciosus (Poepp.) Wiehler Cremosperma peruvianum L.E. Skog Diastema hispidum (DC.) Fritsch Diastema racemiferum Benth. Diastema scabrum (Poepp.) Benth. ex Walp. Diastema tenerrimum (Poepp.) Benth. ex Walp. Diastema urticifolium (Poepp.) Benth. ex Walp. Drymonia candida Hanst.

Drymonia dodsonii (Wiehler) J.L. Clark Drymonia erythroloma (Leeuwenb.) Wiehler Drymonia macrophylla (Oerst.) H.E. Moore Drymonia oxysepala Leeuwenb.

Drymonia pendula (Poepp.) Wiehler Drymonia semicordata (Poepp.) Wiehler Drymonia serrulata (Jacq.) Mart.

Drymonia urceolata Wiehler Drymonia warszewicziana Hanst. Gasteranthus wendlandianus (Hanst.) Wiehler Glossoloma carpishense (J.L. Clark \& I. Salinas) J.L. Clark

Glossoloma herthae (Mansf.) J.L. Clark Glossoloma ichthyoderma (Hanst.) J.L. Clark Glossoloma schultzei (Mansf.) J.L. Clark Gloxinia perennis (L.) Fritsch Gloxinia xanthophylla (Poepp.) Roalson \& Boggan Heppiella ulmifolia (Kunth) Hanst.

Monopyle flava L.E. Skog

Monopyle macrocarpa Benth.

Nautilocalyx pallidus (Sprague) Sprague

Nautilocalyx peruvianus Wiehler

Paradrymonia longifolia (Poepp.) Wiehler

Paradrymonia metamorphophylla (Donn. Sm.)

Wiehler

Parakohleria melastoma (Poepp.) Wiehler
Pouteria petiolata T.D. Penn.

Pouteria procera (Mart.) K. Hammer

Pouteria reticulata (Engl.) Eyma

Pouteria rostrata (Huber) Baehni

Pouteria torta (Mart.) Radlk.

Pouteria vernicosa T.D. Penn.

Sarcaulus brasiliensis (A. DC.) Eyma

\section{Saxifragaceae}

Saxifraga magellanica Poir.

\section{Schizaeaceae}

Schizaea elegans (Vahl) Sw.

\section{Schlegeliaceae}

Schlegelia roseiflora Ducke

\section{Schoepfiaceae}

Schoepfia flexuosa (Ruiz \& Pav.) Schult.

\section{Scrophulariaceae}

Alonsoa acutifolia Ruiz \& Pav.

Alonsoa meridionalis (L. f.) Kuntze

Buddleja americana L.

Buddleja davidii Franch.

Buddleja diffusa Ruiz \& Pav.

Buddleja incana Ruiz \& Pav.

Limosella australis $\mathrm{R}$. Br.

Peltanthera floribunda Benth.

Verbascum virgatum Stokes

\section{Selaginellaceae}

Selaginella exaltata (Kunze) Spring

Selaginella geniculata (C. Presl) Spring

Selaginella haematodes (Kunze) Spring

Selaginella speciosa A. Braun

\section{Simaroubaceae}

Simarouba amara Aubl.

\section{Siparunaceae}

Siparuna aspera (Ruiz \& Pav.) A. DC.

Siparuna bifida (Poepp. \& Endl.) A. DC

Siparuna decipiens (Tul.) A. DC.

Siparuna echinata (Kunth) A. DC.

Siparuna guianensis Aubl.

Siparuna muricata (Ruiz \& Pav.) A. DC.

Siparuna ovalis (Ruiz \& Pav.) A. DC.

Siparuna subinodora (Ruiz \& Pav.) A. DC.

Siparuna thecaphora (Poepp. \& Endl.) A. DC.

Siparuna tomentosa (Ruiz \& Pav.) A. DC.

\section{Smilacaceae}

Smilax domingensis Willd.

Smilax oblongata Sw.

Smilax schomburgkiana Kunth

\section{Solanaceae}

Acnistus arborescens (L.) Schltdl. 
Parakohleria purpurea (Poepp.) Wiehler Pearcea fuscicalyx L.P. Kvist \& L.E. Skog Pearcea purpurea (Poepp.) L.P. Kvist \& L.E. Skog Pearcea strigosa L.P. Kvist \& L.E. Skog Sanango racemosum (Ruiz \& Pav.) Barringer Seemannia sylvatica (Kunth) Hanst. Sinningia elatior (Kunth) Chautems

\section{Gleicheniaceae}

Dicranopteris flexuosa (Schrad.) Underw. Diplopterygium bancroftii (Hook.) A.R. Sm. Gleichenella pectinata (Willd.) Ching Sticherus bifidus (Willd.) Ching Sticherus lechleri (Mett. ex Kuhn) Nakai Sticherus longipinnatus (Hook.) Ching Sticherus nudus (Moritz ex Reichard) Nakai Sticherus peruvianus (Maxon) A.R. Sm., M. Kessler

\& J. Gonzales

Sticherus pruinosus (Mart.) Ching

Sticherus remotus (Kaulf.) Chrysler

Sticherus revolutus (Kunth) Ching

Sticherus rubiginosus (Mett.) Nakai

Sticherus simplex (Desv.) Ching

Sticherus tomentosus (Cav. ex Sw.) A.R. Sm.

\section{Gnetaceae}

Gnetum leyboldii Tul.

\section{Grossulariaceae}

Ribes albifolium Ruiz \& Pav.

Ribes cuneifolium Ruiz \& Pav.

Ribes elegans Jancz.

Ribes macrobotrys Ruiz \& Pav.

Ribes viscosum Ruiz \& Pav.

\section{Gunneraceae}

Gunnera annae Schindl.

Gunnera peruviana J.F. Macbr.

\section{Haemodoraceae}

Xiphidium caeruleum Aubl.

\section{Haloragaceae}

Myriophyllum quitense Kunth

\section{Heliconiaceae}

Heliconia aemygdiana Burle-Marx

Heliconia chartacea Lane ex Barreiros

Heliconia episcopalis Vell.

Heliconia fugax L. Andersson

Heliconia hirsuta L. f.

Heliconia lingulata Ruiz \& Pav.

Heliconia marginata (Griggs) Pittier

Heliconia metallica Planch. \& Linden ex Hook.

Heliconia penduloides Loes.
Browallia americana L.

Browallia speciosa Hook.

Brugmansia sanguinea (Ruiz \& Pav.) D. Don

Brunfelsia grandiflora D. Don

Capsicum annuum L.

Capsicum coccineum (Rusby) Hunz.

Cestrum auriculatum L'HÃ@r.

Cestrum conglomeratum Ruiz \& Pav.

Cestrum humboldtii Francey

Cestrum megalophyllum Dunal

Cestrum nemanthum Dunal

Cestrum racemosum Ruiz \& Pav.

Cestrum rigidum Rusby

Cestrum tomentosum L. f.

Cestrum undulatum Ruiz \& Pav.

Cyphomandra betacea (Cav.) Sendtn.

Cyphomandra endopogon Bitter

Datura stramonium L.

Dunalia spathulata (Ruiz \& Pav.) Braun \& Bouché

lochroma umbellatum (Ruiz \& Pav.) Hunziker ex

D'Arcy

Jaltomata repandidentata (Dunal) Hunz.

Juanulloa parasitica Ruiz \& Pav.

Larnax lutea S. Leiva

Lycianthes amatitlanensis (J.M. Coult. \& Donn. Sm.)

Bitter

Lycianthes lycioides (L.) Hassl.

Lycianthes pauciflora (Vahl) Bitter

Lycianthes poeppigii Bitter

Lycianthes stenoloba (Van Heurck \& Müll. Arg.) Bitter

Lycium americanum Jacq.

Lycopersicon parviflorum C.M. Rick, Kesicki, Fobes \&

M. Holle

Markea ulei (Dammer) Cuatrec.

Nicandra physalodes (L.) Gaertn.

Nicotiana rustica Comes

Nicotiana tabacum L.

Nicotiana tomentosa Ruiz \& Pav.

Physalis angulata $\mathrm{L}$.

Saracha punctata Ruiz \& Pav.

Schultesianthus leucanthus (Donn. Sm.) Hunz.

Sessea dependens Ruiz \& Pav.

Sessea macrophylla Francey

Sessea stipulata Ruiz \& Pav.

Solanum acaule Bitter

Solanum acroglossum Juz.

Solanum acuminatum Ruiz \& Pav.

Solanum albidum Dunal

Solanum ambosinum Ochoa

Solanum americanum Mill. 
Heliconia rostrata Ruiz \& Pav.

Heliconia schumanniana Loes.

Heliconia spathocircinata Aristeg.

Heliconia stricta Huber

Heliconia subulata Ruiz \& Pav.

Heliconia vellerigera Poepp.

Heliconia zebrina Plowman, W.J. Kress \& H. Kenn.

\section{Hemidictyaceae}

Hemidictyum marginatum (L.) C. Presl

\section{Hernandiaceae}

Sparattanthelium amazonum Mart.

\section{Humiriaceae}

Humiriastrum colombianum (Cuatrec.) Cuatrec. Humiriastrum excelsum (Ducke) Cuatrec.

\section{Hydrangeaceae}

Hydrangea diplostemona (Donn. Sm.) Standl.

Hydrangea preslii Briq.

\section{Hydrocharitaceae}

Elodea potamogeton (Bertero) Espinosa

\section{Hymenophyllaceae}

Abrodictyum rigidum (Sw.) Ebihara \& Dubuisson

Hymenophyllum apiculatum Mett. ex Kuhn

Hymenophyllum fucoides (Sw.) Sw.

Hymenophyllum hirsutum (L.) Sw.

Hymenophyllum lobatoalatum Klotzsch

Hymenophyllum matthewsii Bosch

Hymenophyllum microcarpum Desv.

Hymenophyllum myriocarpum Hook.

Hymenophyllum plumieri Hook. \& Grev.

Hymenophyllum plumosum Kaulf.

Hymenophyllum pyramidatum Desv.

Hymenophyllum ruizianum (Klotzsch) Kunze

Hymenophyllum tomentosum Kunze

Hymenophyllum trichomanoides Bosch

Hymenophyllum undulatum (Sw.) Sw.

Hymenophyllum valvatum Hook. \& Grev.

Hymenophyllum verecundum C.V. Morton

Trichomanes angustifrons (Fée) Wess. Boer

Trichomanes ankersii C. Parker ex Hook. \& Grev.

Trichomanes cellulosum Klotzsch

Trichomanes crinitum Sw.

Trichomanes cristatum Kaulf.

Trichomanes diversifrons (Bory) Mett. ex Sadeb.

Trichomanes elegans Rich.

Trichomanes kapplerianum J.W. Sturm

Trichomanes krausii Hook. \& Grev.

Trichomanes lucens Sw.

Trichomanes martiusii C. Presl

Trichomanes membranaceum $\mathrm{L}$.

Trichomanes pinnatum Hedw.
Solanum anceps Ruiz \& Pav.

Solanum aphyodendron S. Knapp

Solanum appressum K.E. Roe

Solanum ariduphilum Ochoa

Solanum asperolanatum Ruiz \& Pav.

Solanum aureum Dunal

Solanum barbeyanum Huber

Solanum barbulatum Zahlbr.

Solanum billhookeri Ochoa

Solanum cacosmum Bohs

Solanum calidum Bohs

Solanum chomatophilum Bitter

Solanum chrysotrichum Schltdl.

Solanum circinatum Bohs

Solanum compressibaccatum Bitter

Solanum confine Dunal

Solanum conicum Ruiz \& Pav.

Solanum cutervanum Zahlbr.

Solanum dolichocremastrum Bitter

Solanum furcatum Dunal

Solanum goniocalyx Juz. \& Bukasov

Solanum grandiflorum Ruiz \& Pav.

Solanum incarceratum Ruiz \& Pav.

Solanum incurvum Ruiz \& Pav.

Solanum insolaesolis Bitter

Solanum laxissimum Bitter

Solanum leucopogon Huber

Solanum lindenii Rusby

Solanum luteoalbum Pers.

Solanum macrotonum Bitter

Solanum mammosum L.

Solanum maturecalvans Bitter

Solanum mite Ruiz \& Pav.

Solanum monadelphum Van Heurck \& Müll. Arg.

Solanum nemorense Dunal

Solanum nitidum Ruiz \& Pav.

Solanum nubicola Ochoa

Solanum nutans Ruiz \& Pav.

Solanum obliquum Ruiz \& Pav.

Solanum oppositifolium Ruiz \& Pav.

Solanum oxycoccoides Bitter

Solanum oxyphyllum C.V. Morton

Solanum parvicorollatum Lekhnovich

Solanum pendulum Ruiz \& Pav.

Solanum probolospermum Bitter

Solanum pseudocapsicum L.

Solanum radicans $\mathrm{L}$. $\mathrm{f}$.

Solanum riparium Pers.

Solanum robustifrons Bitter

Solanum santolallae Vargas 
Trichomanes plumosum Kunze

Trichomanes polypodioides $\mathrm{L}$.

Trichomanes punctatum Poir.

Trichomanes reptans Sw.

Vandenboschia radicans (Sw.) Copel.

\section{Hypericaceae}

Hypericum brevistylum Choisy

Hypericum laricifolium Juss.

Hypericum silenoides Juss.

Hypericum struthiolifolium Juss.

Vismia glabra Ruiz \& Pav.

Vismia gracilis Hieron.

Vismia lateriflora Ducke

Vismia macrophylla Kunth

Vismia pozuzoensis Engl.

Vismia rusbyi Ewan

Vismia sprucei Sprague

Vismia tomentosa Ruiz \& Pav.

\section{Hypoxidaceae}

Hypoxis decumbens $\mathrm{L}$.

\section{Icacinaceae}

Calatola costaricensis Standl.

Leretia cordata Vell.

\section{Iridaceae}

Cipura paludosa Aubl.

Orthrosanthus chimboracensis (Kunth) Baker

Orthrosanthus occissapungus (Ruiz ex Klatt) Diels

Sisyrinchium caespitificum Kraenzl.

Sisyrinchium chilense Hook.

Sisyrinchium convolutum Nocca

Sisyrinchium palmifolium L.

\section{Isoetaceae}

Isoetes andicola (Amstutz) L.D. Gómez

\section{Juglandaceae}

Juglans neotropica Diels

\section{Juncaceae}

Distichia muscoides Nees \& Meyen

Juncus arcticus Willd.

Juncus bufonius $\mathrm{L}$.

Juncus dudleyi Wiegand

Juncus ebracteatus E. Mey.

Juncus imbricatus Laharpe

Juncus microcephalus Kunth

Juncus pallescens Lam.

Luzula vulcanica Liebm.

\section{Juncaginaceae}

Triglochin scilloides (Poir.) Mering \& Kadereit

\section{Krameriaceae}

Krameria lappacea (Dombey) Burdet \& B.B.
Solanum saponaceum Dunal

Solanum scabrifolium Ochoa

Solanum selachophyllum Bitter

Solanum semiscandens Bitter

Solanum sessile Ruiz \& Pav.

Solanum sessiliflorum Dunal

Solanum stramoniifolium Jacq.

Solanum suaveolens Kunth \& C.D. Bouché

Solanum subinerme Jacq.

Solanum tenuisetosum (Bitter) Bohs

Solanum ternatum Ruiz \& Pav.

Solanum theobromophyllum Bitter

Solanum trachycyphum Bitter

Solanum trichoneuron Lillo

Solanum tuberosum $\mathrm{L}$.

Solanum uleanum Bitter

Solanum umbellatum Mill.

Solanum valdiviense Dunal

Solanum xanthophaeum Bitter

Trianaea nobilis Planch. \& Linden

Witheringia solanacea L'HÃ@r.

\section{Staphyleaceae}

Turpinia occidentalis (Sw.) G. Don

\section{Stemonuraceae}

Discophora guianensis Miers

\section{Styracaceae}

Styrax argenteus C. Presl

Styrax foveolaria Perkins

Styrax oblongus (Ruiz \& Pav.) A. DC.

Styrax omuk B. Walln.

Styrax pavonii A. DC.

\section{Symplocaceae}

Symplocos coriacea A. DC.

Symplocos globulifera B. Ståhl

Symplocos scabra J.F. Macbr.

\section{Talinaceae}

Talinum paniculatum (Jacq.) Gaertn.

\section{Tapisciaceae}

Huertea glandulosa Ruiz \& Pav.

\section{Tectariaceae}

Tectaria draconoptera (D.C. Eaton) Copel.

Tectaria heracleifolia (Willd.) Underw.

Tectaria incisa Cav.

\section{Theaceae}

Gordonia fruticosa (Schrad.) H. Keng

\section{Thelypteridaceae}

Cyclosorus consobrinus (Maxon \& C.V. Morton)

Mazumdar \& R.Mukhop.

Cyclosorus depilatus (A.R. Sm.) Mazumdar \&

R.Mukhop.

Cyclosorus semihastatus (Kunze) Mazumdar \& 


\begin{tabular}{|l|}
\hline Simpson \\
\hline Lacistemataceae \\
\hline Lacistema aggregatum (P.J. Bergius) Rusby \\
\hline Lacistema nena J.F. Macbr. \\
\hline Lozania klugii (Mansf.) Mansf. \\
\hline Lamiaceae \\
\hline Aegiphila alba Moldenke \\
\hline Aegiphila cordata Poepp. \\
\hline Aegiphila cordifolia (Ruiz \& Pav.) Moldenke \\
\hline Aegiphila glabrata Moldenke \\
\hline Aegiphila insignis Moldenke \\
\hline Aegiphila integrifolia (Jacq.) B.D. Jacks. \\
\hline Aegiphila multiflora Ruiz \& Pav. \\
\hline Aegiphila sordida Moldenke \\
\hline Aegiphila spicata (Rusby) Moldenke \\
\hline Aegiphila triflora Moldenke \\
\hline Aegiphila ulei (Hayek) B. Walln. \\
\hline Aegiphila umbraculiformis Moldenke \\
\hline Aegiphila vitelliniflora Klotzsch ex Walp. \\
\hline Aegiphila volubilis Moldenke \\
\hline Cantinoa americana (Aubl.) Harley \& J.F.B. Pastore \\
\hline Cantinoa colombiana (Epling) Harley \& J.F.B. \\
\hline Pastore \\
\hline Catoferia spicata (Benth.) Benth. \\
\hline Clinopodium argenteum (Kunth) Govaerts \\
\hline Clinopodium breviflorum (Benth.) Govaerts \\
\hline Clinopodium nubigenum (Kunth) Kuntze \\
\hline Clinopodium plicatulum (Epling) Govaerts \\
\hline Clinopodium sericeum (C. Presl ex Benth.) Govaerts \\
\hline Clinopodium striatum (Ruiz \& Pav.) Govaerts \\
\hline Condea tafallae (Benth.) Harley \& J.F.B. Pastore \\
\hline Hyptidendron arboreum (Benth.) Harley \\
\hline Hyptis brevipes Poit. \\
\hline Hyptis conferta Pohl ex Benth. \\
\hline Hyptis lantanifolia Poit. \\
\hline Hyptis lutescens Pohl ex Benth. \\
\hline Hyptis mutabilis (Rich.) Briq. \\
\hline Hyptis obtusiflora C. Presl ex Benth. \\
\hline Hyptis odorata Benth. \\
\hline Hyptis sidifolia (L'HÃ@r.) Briq. \\
\hline Hyptis uncinata Benth. \\
\hline Lamium amplexicaule L. \\
\hline Lepechinia meyenii (Walp.) Epling \\
\hline Marrubium vulgare L. \\
\hline Mesosphaerum obtusatum (Benth.) Kuntze \\
\hline Sinthostachys mollis (Kunth) Griseb. \\
\hline Salvia cuspidatata Ruiz \& Pav. Pav. \\
\hline
\end{tabular}

R.Mukhop.

Macrothelypteris torresiana (Gaudich.) Ching

Thelypteris biformata (Rosenst.) R.M. Tryon

Thelypteris decussata (L.) Proctor

Thelypteris demissa A.R. Sm.

Thelypteris furva (Maxon) R.M. Tryon

Thelypteris gigantea (Mett.) R.M. Tryon

Thelypteris jamesonii (Hook.) R.M. Tryon

Thelypteris pusilla (Mett.) Ching

Thelypteris rudis (Kunze) Proctor

Thelypteris rufa (Poir.) A.R. Sm.

Thelypteris ruiziana (Klotzsch) A.R. Sm.

Thelypteris serrata (Cav.) Alston

\section{Thymelaeaceae}

Schoenobiblus daphnoides Mart.

Schoenobiblus peruvianus Standl.

\section{Tofieldiaceae}

Isidrogalvia falcata Ruiz \& Pav.

\section{Tovariaceae}

Tovaria pendula Ruiz \& Pav.

\section{Trigoniaceae}

Trigonia killipii J.F. Macbr.

\section{Tropaeolaceae}

Tropaeolum bicolor Ruiz \& Pav.

Tropaeolum crenatiflorum Hook.

Tropaeolum dipetalum Ruiz \& Pav.

Tropaeolum peregrinum $\mathrm{L}$.

Tropaeolum tuberosum Ruiz \& Pav.

\section{Ulmaceae}

Ampelocera edentula Kuhlm.

\section{Urticaceae}

Boehmeria aspera Wedd.

Boehmeria brevirostris Wedd.

Boehmeria bullata Kunth

Boehmeria caudata Sw.

Boehmeria pavonii Wedd.

Boehmeria ramiflora Jacq.

Boehmeria ulmifolia Wedd.

Cecropia albicans Trécul

Cecropia andina Cuatrec.

Cecropia angustifolia Trécul

Cecropia dentata Klotzsch

Cecropia engleriana Snethl.

Cecropia membranacea Trécul

Cecropia montana Warb. ex Snethl.

Cecropia polystachya Trécul

Cecropia sciadophylla Mart.

Cecropia strigosa Trécul

Coussapoa ovalifolia Trécul 
Salvia incurvata Ruiz \& Pav.

Salvia lachnostoma Epling

Salvia macrophylla Benth.

Salvia occidentalis Sw.

Salvia oppositiflora Ruiz \& Pav.

Salvia penduliflora Epling

Salvia plumosa Ruiz \& Pav.

Salvia punctata Ruiz \& Pav.

Salvia revoluta Ruiz \& Pav.

Salvia rivularis Gardner

Salvia sagittata Ruiz \& Pav.

Salvia scutellarioides Kunth

Salvia speciosa C. Presl ex Benth.

Scutellaria aurata Lem.

Scutellaria coccinea Kunth

Scutellaria hookeri Epling

Scutellaria irrasa Epling

Stachys aperta Epling

Stachys gilliesii Benth.

Stachys pusilla (Wedd.) Briq.

Vitex triflora Vahl

\section{Lauraceae}

Aniba guianensis Aubl.

Aniba muca (Ruiz \& Pav.) Mez

Aniba taubertiana Mez

Cinnamomum triplinerve (Ruiz \& Pav.) Kosterm.

Endlicheria bracteata Mez

Endlicheria dysodantha (Ruiz \& Pav.) Mez

Endlicheria paniculata (Spreng.) J.F. Macbr.

Endlicheria sericea Nees

Endlicheria tessmannii O.C. Schmidt

Nectandra acutifolia (Ruiz \& Pav.) Mez

Nectandra cissiflora Nees

Nectandra cuspidata Nees \& Mart.

Nectandra furcata (Ruiz \& Pav.) Nees

Nectandra japurensis Nees \& C. Mart.

Nectandra lineatifolia (Ruiz \& Pav.) Mez

Nectandra longifolia (Ruiz \& Pav.) Nees

Nectandra matthewsii Meisn.

Nectandra maynensis Mez

Nectandra membranacea (Sw.) Griseb.

Nectandra purpurea (Ruiz \& Pav.) Mez

Nectandra reticulata (Ruiz \& Pav.) Mez

Nectandra turbacensis (Kunth) Nees

Ocotea aciphylla (Nees \& Mart.) Mez

Ocotea amplissima Mez

Ocotea aurantiodora (Ruiz \& Pav.) Mez

Ocotea cardinalis Mez

Ocotea cuneifolia (Ruiz \& Pav.) Mez

Ocotea monzonensis $\mathrm{Mez}$
Coussapoa villosa Poepp. \& Endl.

Myriocarpa laevigata Killip

Myriocarpa stipitata Benth.

Phenax angustifolius (Kunth) Wedd.

Phenax hirtus (Sw.) Wedd.

Phenax laevigatus Wedd.

Phenax laxiflorus Wedd.

Pilea ceratocalyx Wedd.

Pilea citriodora Wedd.

Pilea cushiensis Killip

Pilea dauciodora Wedd.

Pilea delicatula Killip

Pilea diversifolia Wedd.

Pilea dombeyana Wedd.

Pilea fendleri Killip

Pilea haenkei Killip

Pilea hyalina Fenzl

Pilea macbridei Killip

Pilea marginata Wedd.

Pilea microphylla (L.) Liebm.

Pilea multiflora (Poir.) Wedd.

Pilea nerteroides Killip

Pilea nutans Wedd.

Pilea pavonii Wedd.

Pilea poeppigiana Wedd.

Pilea pulegifolia (Poir.) Wedd.

Pilea pusilla K. Krause

Pilea ramosissima Killip

Pilea spruceana Wedd.

Pilea strigosa Wedd.

Pilea verrucosa Killip

Pourouma bicolor Mart.

Pourouma cecropiifolia Mart.

Pourouma cucura Standl. \& Cuatrec.

Pourouma guianensis Aubl.

Pourouma minor Benoist

Pourouma mollis Trécul

Pouzolzia poeppigiana (Wedd.) Killip

Urera baccifera (L.) Gaudich. ex Wedd.

Urera caracasana (Jacq.) Gaudich. ex Griseb.

Urera laciniata Wedd.

Urera simplex Wedd.

Urtica echinata Benth.

Urtica flabellata Kunth

Urtica macbridei Killip

Urtica magellanica Juss. ex Poir.

\section{Verbenaceae}

Aloysia scorodonioides (Kunth) Cham.

Bouchea fluminensis (Vell.) Moldenke

Citharexylum flexuosum (Ruiz \& Pav.) D. Don 
Ocotea munacensis O.C. Schmidt Ocotea obovata (Ruiz \& Pav.) Mez Ocotea ovalifolia (Ruiz \& Pav.) Mez Ocotea raimondii O.C. Schmidt Persea caerulea (Ruiz \& Pav.) Mez Persea hexanthera L.E. Kopp

Persea peruviana Nees

Persea ruizii J.F. Macbr.

Persea subcordata (Ruiz \& Pav.) Nees

Pleurothyrium tomentellum van der Werff

Rhodostemonodaphne grandis (Mez) Rohwer

Rhodostemonodaphne synandra van der Werff

\section{Lecythidaceae}

Bertholletia excelsa Bonpl.

Couroupita guianensis Aubl.

Eschweilera albiflora (DC.) Miers

Eschweilera andina (Rusby) J.F. Macbr.

Eschweilera bracteosa (Poepp. ex O. Berg) Miers

Grias peruviana Miers

\section{Lentibulariaceae}

Pinguicula involuta Ruiz \& Pav.

Utricularia jamesoniana Oliv.

Utricularia unifolia Ruiz \& Pav.

\section{Lindsaeaceae}

Lindsaea guianensis (Aubl.) Dryand.

Lindsaea hemiglossa K.U. Kramer

\section{Loasaceae}

Caiophora carduifolia C. Presl

Caiophora cirsiifolia C. Presl

Caiophora contorta (Desr.) C. Presl

Caiophora grandiflora (Ruiz \& Pav. ex G. Don)

Weigend \& Mark. Ackermann

Caiophora macrantha Killip

Caiophora tenuis Killip

Klaprothia fasciculata (C. Presl) Poston

Klaprothia mentzelioides Bonpl. \& Kunth

Mentzelia scabra Kunth

Nasa lenta (J.F. Macbr.) Weigend

Nasa macrantha (Urb. \& Gilg) Weigend

Nasa rugosa (Killip) Weigend

Nasa tingomariensis (J.F. Macbr.) Weigend

Nasa vargasii (J.F. Macbr.) Weigend

\section{Loganiaceae}

Spigelia anthelmia L.

Strychnos amazonica Krukoff

Strychnos colombiensis Krukoff \& Barneby

Strychnos guianensis (Aubl.) Mart.

Strychnos jobertiana Baill.

Strychnos peckii B.L. Rob.

Strychnos poeppigii Progel
Citharexylum reticulatum Kunth

Citharexylum weberbaueri Hayek

Duranta armata Moldenke

Duranta dombeyana Moldenke

Duranta mutisii L. f.

Duranta obtusifolia Kunth

Duranta peruviana Moldenke

Duranta skottsbergii Moldenke

Duranta sprucei Briq.

Glandularia clavata (Ruiz \& Pav.) Botta

Glandularia laciniata (L.) Schnack \& Covas

Lantana camara L.

Lantana cujabensis Schauer

Lantana haughtii Moldenke

Lantana hirsuta M. Martens \& Galeotti

Lantana horrida Kunth

Lantana rugulosa Kunth

Lantana scabiosiflora Kunth

Lantana trifolia L.

Lantana weberbaueri Hayek

Lantana zahlbruckneri Hayek

Lippia alba (Mill.) N.E. Br. ex Britton \& P. Wilson

Petrea maynensis Huber

Phyla nodiflora (L.) Greene

Priva lappulacea (L.) Pers.

Stachytarpheta cayennensis (Rich.) Vahl

Verbena cuneifolia Ruiz \& Pav.

Verbena hispida Ruiz \& Pav.

Verbena litoralis Kunth

\section{Violaceae}

Hybanthus attenuatus (Humb. \& Bonpl. ex Willd.)

Schulze-Menz

Hybanthus parviflorus (L. f.) Baill.

Leonia crassa L.B. Sm. \& A. Fernández

Leonia glycycarpa Ruiz \& Pav.

Leonia racemosa Mart.

Noisettia orchidiflora (Rudge) Ging.

Rinorea apiculata Hekking

Rinorea falcata (Mart. ex Eichler) Kuntze

Rinorea flavescens (Aubl.) Kuntze

Rinorea lindeniana (Tul.) Kuntze

Rinorea viridifolia Rusby

Viola arguta Willd. ex Roem. \& Schult.

Viola boliviana Britton

Viola membranacea W. Becker

\section{Vitaceae}

Cissus alata Jacq.

Cissus bracteosa Lombardi

Cissus erosa Rich.

Cissus granulosa Ruiz \& Pav. 
Strychnos tarapotensis Sprague \& Sandwith

\section{Lomariopsidaceae}

Cyclopeltis semicordata (Sw.) J. Sm.

Lomariopsis japurensis (Mart.) J. Sm.

Lomariopsis latipinna Stolze

\section{Loranthaceae}

Aetanthus paxianus Patsch.

Cladocolea lenticellata (Diels) Kuijt

Gaiadendron punctatum (Ruiz \& Pav.) G. Don

Ligaria cuneifolia (Ruiz \& Pav.) Tiegh.

Oryctanthus alveolatus (Kunth) Kuijt

Oryctanthus florulentus (Rich.) Tiegh.

Oryctanthus ovalifolius (Ruiz \& Pav.) J.F. Macbr.

Passovia pedunculata (Jacq.) Kuijt

Passovia pyrifolia (Kunth) Tiegh.

Peristethium polystachyum (Ruiz \& Pav.) Kuijt

Psittacanthus krausei J.F. Macbr.

Psittacanthus subalatus K. Krause

Psittacanthus zonatus (Diels) Kuijt

Struthanthus concinnus Mart.

Struthanthus orbicularis (Kunth) Blume

Struthanthus sarmentosus (Ruiz \& Pav.) Blume

Tripodanthus acutifolius (Ruiz \& Pav.) Tiegh.

Tristerix grandiflorus (Ruiz \& Pav.) Barlow \& Wiens

Tristerix longebracteatus (Desr.) Barlow \& Wiens

Loxsomataceae

Loxsomopsis pearcei (Baker) Maxon

Lycopodiaceae

Phlegmariurus andinus (Rosenst.) B. Øllg.

Phlegmariurus engleri (Herter) B. Øllg.

\section{Lygodiaceae}

Lygodium venustum Sw.
Cissus microcarpa Vahl

Cissus obliqua Ruiz \& Pav.

Cissus peruviana Lombardi

Cissus ulmifolia (Baker) Planch.

Cissus verticillata (L.) Nicolson \& C.E. Jarvis

\section{Vochysiaceae}

Qualea grandiflora Mart.

Qualea paraensis Ducke

Qualea tessmannii Mildbr.

Vochysia lomatophylla Standl.

\section{Xanthorrhoeaceae}

Eccremis coarctata (Ruiz \& Pav.) Baker

\section{Xyridaceae}

Xyris confusa L.B. Sm. \& Downs

Xyris macbrideana L.B. Sm. \& Downs

Xyris subulata Ruiz \& Pav.

\section{Zamiaceae}

Zamia poeppigiana Mart. \& Eichler

Zamia urep B. Walln.

\section{Zingiberaceae}

Renealmia breviscapa Poepp. \& Endl.

Renealmia cernua (Sw. ex Roem. \& Schult.) J.F.

Macbr.

Renealmia monosperma Miq.

Renealmia nicolaioides Loes.

Renealmia racemosa Poepp. \& Endl.

Renealmia thyrsoidea (Ruiz \& Pav.) Poepp. \& Endl.

\section{Zygophyllaceae}

Kallstroemia parviflora Norton

Porlieria hygrometra Ruiz \& Pav.

Tribulus terrestris $\mathrm{L}$. 